\title{
Ex-Core Radiation Transport Modeling with VERA User Manual
}

Tara Pandya, ORNL Thomas Evans, ORNL Katherine Royston, ORNL Kevin Clarno, formerly ORNL Benjamin Collins, ORNL Shane Stimpson, ORNL Shane Henderson, ORNL

September 30, 2019
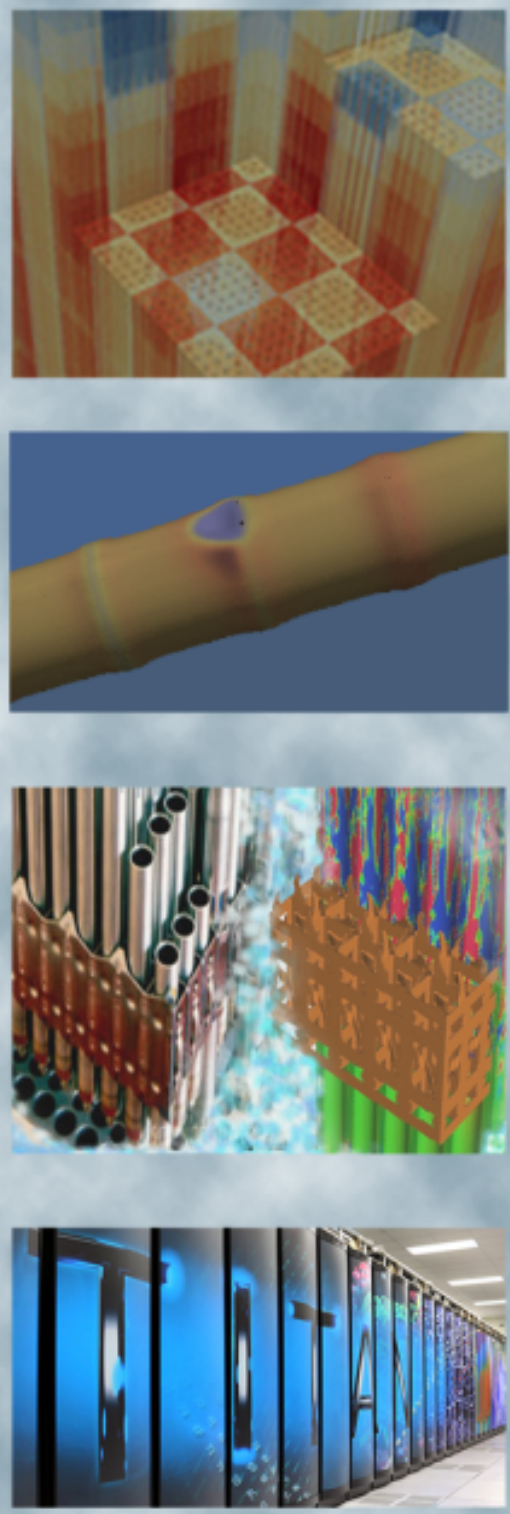

Approved for public release

Distribution is unlimited 


\section{DOCUMENT AVAILABILITY}

Reports produced after January 1, 1996, are generally available free via US Department of Energy (DOE) SciTech Connect.

Website www.osti.gov

Reports produced before January 1, 1996, may be purchased by members of the public from the following source:

National Technical Information Service

5285 Port Royal Road

Springfield, VA 22161

Telephone 703-605-6000 (1-800-553-6847)

TDD 703-487-4639

Fax 703-605-6900

E-mail info@ntis.gov

Website http://classic.ntis.gov/

Reports are available to DOE employees, DOE contractors, Energy Technology Data Exchange representatives, and International Nuclear Information System representatives from the following source:

Office of Scientific and Technical Information

PO Box 62

Oak Ridge, TN 37831

Telephone 865-576-8401

Fax 865-576-5728

E-mail reports@osti.gov

Website http://www.osti.gov/contact.html

This report was prepared as an account of work sponsored by an agency of the United States Government. Neither the United States Government nor any agency thereof, nor any of their employees, makes any warranty, express or implied, or assumes any legal liability or responsibility for the accuracy, completeness, or usefulness of any information, apparatus, product, or process disclosed, or represents that its use would not infringe privately owned rights. Reference herein to any specific commercial product, process, or service by trade name, trademark, manufacturer, or otherwise, does not necessarily constitute or imply its endorsement, recommendation, or favoring by the United States Government or any agency thereof. The views and opinions of authors expressed herein do not necessarily state or reflect those of the United States Government or any agency thereof. 
REVISION LOG

\begin{tabular}{|c|c|c|l|}
\hline Revision & Date & Affected Pages & \multicolumn{1}{|c|}{ Revision Description } \\
\hline 0 & $4 / 19 / 2018$ & All & Initial Public Release - Unlimited \\
\hline 1 & $9 / 2018$ & All & Not publicly released (updates for VERA 4.0) \\
\hline 2 & $10 / 2019$ & All & Updates for VERA 4.0.1 \\
\hline & & & \\
\hline
\end{tabular}

\section{Document pages that are:}

Export Controlled ___ None

IP/Proprietary/NDA Controlled____ None

Sensitive Controlled___ None

\section{Requested Distribution:}

To:

Bill Martin

Dave Kropaczek

Copy:

Jeff Banta

Andrew Godfrey 


\section{EXECUTIVE SUMMARY}

It is important to be able to accurately predict the neutron flux outside the immediate reactor core for a variety of safety and material analyses. Monte Carlo radiation transport calculations are required to produce these high-fidelity ex-core responses. The Virtual Environment for Reactor Applications (VERA) provides the automated capability to launch independent Shift [1] fixed-source and eigenvalue Monte Carlo (MC) calculations for user-specified state points during a standard Virtual Environment for Reactor Applications (VERA) calculation. VERA couples MPACT with COBRA-TF (CTF) to Shift to perform ex-core tallies for multiple state points concurrently, with each component capable of parallel execution on independent processor domains.

In these ex-core calculations, MPACT is coupled to CTF and performs the in-core depletion and heat transfer calculation, followed by a fixed-source Shift transport calculation including ex-core regions to produce ex-core responses. The fission source, fuel pin temperatures, moderator temperature and density, boron concentration, and fuel pin depleted isotopic compositions can be transferred to Shift from the MPACT calculation. Specifically, VERA performs fluence calculations in the core barrel outward to the end of the pressure vessel and detector response calculations in ex-core detectors. It also performs the requested tallies in any user-defined ex-core regions.

VERA takes advantage of the General Geometry (GG) package in Shift. This gives VERA the flexibility to explicitly model features outside the core barrel, including detailed vessel models, detectors, and power plant details. A very limited set of experimental and numerical benchmarks is available for ex-core simulation comparison. The Consortium for Advanced Simulation of Light Water Reactors has developed a set of ex-core benchmark problems to include as part of the VERA verification and validation set of problems. The ex-core capability in VERA has been tested on small representative assembly problems, multi-assembly problems, as well as quarter-core and full-core problems. VERAView has also been extended to visualize these vessel fluence results from VERA.

This manual serves to present a guide to VERA users about the methodology behind ex-core calculations and the details of input, output, and analysis of results from these calculations. Details in this version of the manual are based on features in VERA 4.0.1. 


\section{DEVELOPER TEAM}

The following people are contributors to the development of the specific parts of VERA relevant for ex-core calculations.

\section{Shift Team}

Elliott Biondo

Gregory Davidson

Thomas Evans

Seth Johnson

Steven Hamilton

Tara Pandya

Katherine Royston

Jose Salcedo-Perez

Casey Stocking
ORNL

ORNL

ORNL

ORNL

ORNL

ORNL

ORNL

ORNL

(formerly NCSU)

\section{MPACT Team}

Benjamin Collins (also PHI) ORNL

Shane Stimpson

ORNL

Shane Henderson

ORNL

\section{PHI Team}

Kevin Clarno

Stuart Slattery

University of Texas (formerly ORNL)

ORNL

\section{CTF Team}

Robert Salko

ORNL

\section{INF Team}

Mark Baird

Ron Lee

ORNL

ORNL 


\section{CONTENTS}

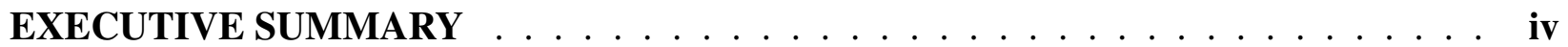

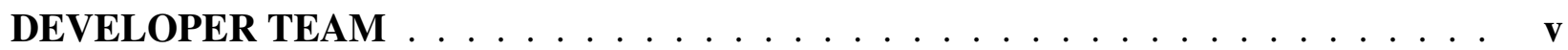

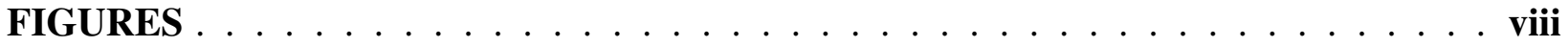

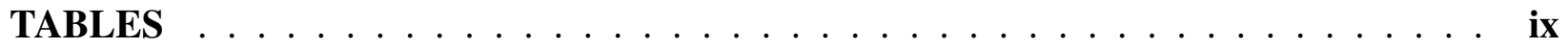

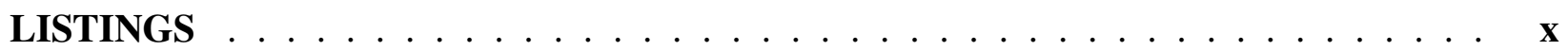

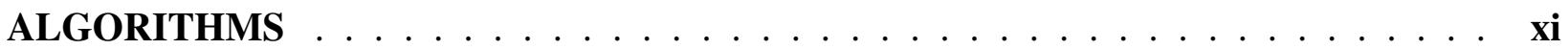

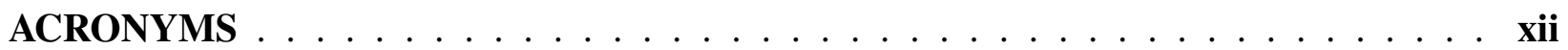

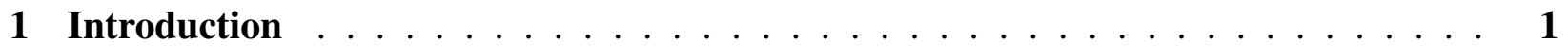

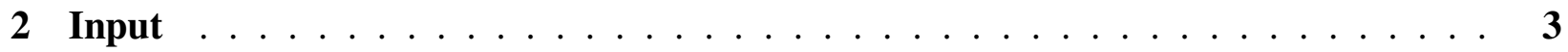

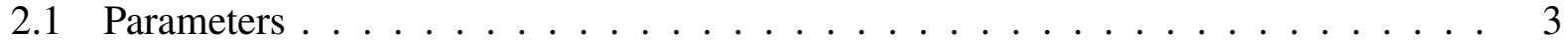

2.2 Example Shift input blocks . . . . . . . . . . . . . . . . 10

3 Running . . . . . . . . . . . . . . . . . . . . . 12

3.1 Running with verarun . . . . . . . . . . . . . . . . . . . . . 12

3.2 Running manually . . . . . . . . . . . . . . . . . . . . 13

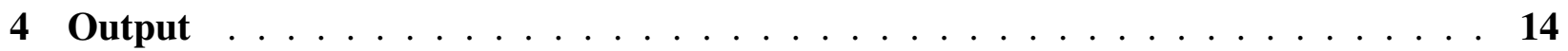

4.1 General output . . . . . . . . . . . . . . . . . . . . . . . 14

4.2 Eigenvalue calculation . . . . . . . . . . . . . . . . . . . 14

4.3 Simple vessel fluence calculation . . . . . . . . . . . . . . . . . . . . . . . . . . . . 18

4.4 Detector response calculation . . . . . . . . . . . . . . . . . . . . . 18

4.5 Visualization with VERAView . . . . . . . . . . . . . . . . 20

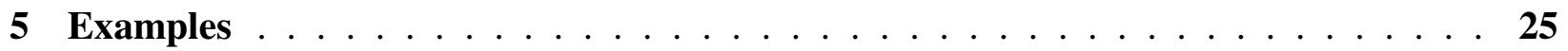

5.1 Shift eigenvalue assembly example . . . . . . . . . . . . . . 25

5.2 Shift forward minicore example . . . . . . . . . . . . . . . . . . . 29

5.3 CADIS vessel fluence minicore example . . . . . . . . . . . . . . . 35

5.4 Automated detectors example . . . . . . . . . . . . . . . . . 37

5.5 Ex-core file detector example . . . . . . . . . . . . . . . . . . . 39

6 Advanced and Experimental Features . . . . . . . . . . . . . . . . . 51

6.1 Core Temperature Homogenization . . . . . . . . . . . . . . . . . 51

6.2 Advanced postprocessing . . . . . . . . . . . . . . . . . 51

7 Summary .......................... 61

8 Acknowledgments ........................ 62

Consortium for Advanced Simulation of LWRs ni CASL-U-2018-1556-002 
A Omnibus Materials Template File $\ldots \ldots \ldots \ldots \ldots \ldots$

B Defaults $\ldots \ldots \ldots \ldots \ldots \ldots \ldots \ldots \ldots \ldots \ldots \ldots \ldots$

C Geometry .......................... 67

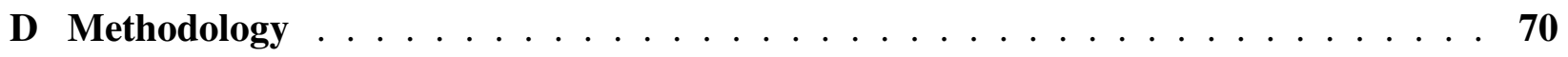

D.1 Package Overview . . . . . . . . . . . . . . . . . . . . 70

D.2 Coupling Algorithm . . . . . . . . . . . . . . . . . . . 73 


\section{FIGURES}

$1 \quad$ VERAView snapshot window of binned neutron flux for progression problem 9. . . 22

2 VERAView snapshot window of binned neutron fluence for progression problem 9.23

3 VERAView snapshot window of pin adjoint for progression problem 9. . . . . . . . 24

4 Ray trace of the progression problem 3a geometry at the axial midplane. . . . . . . 29

5 Ray trace of progression problem 9 geometry at axial midplane. . . . . . . . . . 36

6 Ray trace of example geometry using the automated generation of the supplemental ex-core file with bioshield and detectors. . . . . . . . . . . . . . . . 38

7 Ray trace of example at axial midplane with the ex-core regions defined manually with the supplemental ex-core input file. . . . . . . . . . . . . . . . 50

8 Example of assembly-averaged temperature homogenization in the fuel of progression problem 7 minicore [2] . . . . . . . . . . . . . . . . . . . . . . 52

9 Examples of ring-averaged temperature homogenization in the fuel of progression problem 7 minicore [2]. . . . . . . . . . . . . . . . . . . . . . . 52

10 Example of explicit peripheral pins with assembly-averaged temperature homogenization in the fuel of progression problem 7 minicore [2] $\ldots \ldots$. . . . . . 53

11 Full-core material ray trace $x-y$ slice at axial midplane. . . . . . . . . . . . . . . 54

12 Full-core material ray trace $\mathrm{x}-\mathrm{z}$ slice at $\mathbf{y}=\mathbf{0} \ldots \ldots \ldots 5$

13 Irradiation capsules cell ray trace $\mathrm{x}-\mathrm{y}$ slice. . . . . . . . . . . . . . . . 55

14 Source detector cell ray trace $\mathrm{x}-\mathrm{y}$ slice. . . . . . . . . . . . . . . . 56

15 Progression problem 9 state 2 fission source $x-y$ slice . . . . . . . . . . . . . 57

16 Progression problem 9 adjoint source $\mathrm{x}-\mathrm{y}$ slice. . . . . . . . . . . . . . . 58

17 Progression problem 9 slowest energy group adjoint flux $\mathrm{x}-\mathrm{y}$ slice. . . . . . . . . . 59

18 Progression problem 9 fastest energy group weight window lower bounds $x-y$ slice. 60

19 Performance of Exnihilo General Geometry (GG) compared with Reactor ToolKit (RTK) on reactor geometries with continuous energy (CE) physics. . . . . . . . . . 67

20 Geometry origin relationships in VERA between Shift GG, RTK, and MPACT. In this figure, VERA refers to MPACT. . . . . . . . . . . . . . . . . . 69 


\section{TABLES}

1 SHIFT block inputs related to transport and ex-core through VERA. . . . . . . . 5

2 SHIFT block inputs related to hybrid MC/deterministic through VERA. . . . . . . 6

3 SHIFT block inputs related to hybrid MC/deterministic (continued) and output through VERA. . . . . . . . . . . . . . . . . . . 7

4 SHIFT block inputs related to eigenvalue, physics, and tallies through VERA. . . . 8

5 Shift-related input parameters in the CORE block. . . . . . . . . . . . . 9

6 Experimental temperature homogenization SHIFT block input parameters. . . . . . 9

7 Adjoint flux data fields in the Shift hierarchical data format 5 (HDF5) output file. . 16

8 Fission source data fields in the HDF5 output file. . . . . . . . . . . . . 16

9 Vessel tally data fields in the Shift HDF5 output file. (All data fields are stored in the /STATE_nnnn/tally/vessel_tally group.) . . . . . . . . . . . . . 18

10 Cell tally data fields in the Shift HDF5 output file. (All cell tallies are stored in the /STATE_nnnn/tally group.) . . . . . . . . . . . . . . . 20

11 Ex-core capabilities of VERA. . . . . . . . . . . . . . . . . . . . . . . . . . . . . . . .

12 Nuclide Watt energy spectra constants used in Shift. . . . . . . . . . . . . . 75 


\section{LISTINGS}

1 Required STATE parameter. . . . . . . . . . . . . . . . . 3

2 Example SHIFT block with vessel tally in VERA common input. . . . . . . . . . 10

3 Example SHIFT block with hybrid parameters and vessel tally in VERA common input. . . . . . . . . . . . . . . . . . . . . . 11

4 Example SHIFT block with ex-core parameters in VERA common input. . . . . . . 11

5 Example processor specification for CADIS ex-core calculation using 48 total processors. . . . . . . . . . . . . . . . . . . . . 12

6 Example processor specification for forward ex-core calculation with verarun on 64 total processors. . . . . . . . . . . . . . . . . . . . 13

7 General data in Shift HDF5 output file. . . . . . . . . . . . . . . . . . . 15

8 General data in Shift HDF5 output file (continued). . . . . . . . . . . . . . . 16

9 Data in Shift HDF5 output file from an eigenvalue calculation. . . . . . . . . . . 17

10 Data in Shift HDF5 output file from a vessel fluence calculation . . . . . . . . . 19

11 VERA Consortium for Advanced Simulation of Light Water Reactors (CASL) progression problem 3a Shift eigenvalue input. . . . . . . . . . . . . . 25

12 VERA CASL progression problem 9 minicore input. . . . . . . . . . . . . . . 29

13 VERA CASL progression problem 9 minicore Consistent Adjoint Driven Importance Sampling (CADIS) input. . . . . . . . . . . . . . . . . . . 36

14 VERA CASL progression problem 9 minicore input with automated excore detectors. 37

15 VERA CASL progression problem 5 minicore VERA input using ex-core file. . . . 40

16 VERA CASL progression problem 5 minicore ex-core file input. . . . . . . . . . 48

17 Omnibus materials template file in VERA. . . . . . . . . . . . . . . . . . . . 64

18 Default SHIFT block parameters. . . . . . . . . . . . . . . . . 66 


\section{ALGORITHMS}

1 Simplified VERAShift simulation flow. . . . . . . . . . . . . . . . 74 


\section{ACRONYMS}

$\begin{array}{ll}\text { CADIS } & \text { Consistent Adjoint Driven Importance Sampling } \\ \text { CASL } & \text { Consortium for Advanced Simulation of Light Water Reactors } \\ \text { CE } & \text { continuous energy } \\ \text { CTF } & \text { COBRA-TF } \\ \text { DTK } & \text { Data Transfer Kit } \\ \text { EFPD } & \text { effective full-power days } \\ \text { FW-CADIS } & \text { Forward-Weighted Consistent Adjoint Driven Importance Sampling } \\ \text { GG } & \text { Exnihilo General Geometry } \\ \text { HDF5 } & \text { hierarchical data format 5 } \\ \text { INF } & \text { infrastructure } \\ \text { MC } & \text { Monte Carlo } \\ \text { MG } & \text { multigroup } \\ \text { MPI } & \text { message passing interface } \\ \text { ORNL } & \text { Oak Ridge National Laboratory } \\ \text { PBS } & \text { portable batch system } \\ \text { PHI } & \text { physics integration } \\ \text { PWR } & \text { pressurized water reactor } \\ \text { RTK } & \text { Reactor ToolKit } \\ \text { VERA } & \text { Virtual Environment for Reactor Applications }\end{array}$




\section{INTRODUCTION}

This manual describes the use of and some basic coupling in VERA [3] for ex-core capabilities. VERA provides the automated capability to launch independent Shift [1] fixed-source and eigenvalue MC calculations for user-specified state points during a standard VERA calculation. VERA couples MPACT [4] with COBRA-TF (CTF) [5] to Shift to perform ex-core tallies for multiple state points concurrently, with each component capable of parallel execution on independent processor domains. In these ex-core calculations, MPACT is coupled to CTF and performs the in-core depletion and heat transfer calculation, followed by a fixed-source Shift transport calculation on the entire geometry including ex-core regions to produce ex-core responses. The fission source, fuel pin temperatures, moderator temperature and density, boron concentration, and fuel pin depleted isotopic compositions can be transferred to Shift from the MPACT calculation (if sufficient memory is available). For quarter core and full core problems typically only the fission source and boron concentration are transferred.

For default vessel fluence calculations, minimal user intervention outside the common VERA input is required [6]. This is also true for certain default ex-core detector response calculations in the pressure vessel and bioshield. For more detailed ex-core calculations, ex-core modeling in VERA provides users with the capability to insert a detailed core model into a broader user-defined ex-core model. For example, a detailed core model can be inserted into a containment geometry that includes nozzles and detector locations.

For ex-core calculations, Shift can be run in analog or CADIS [7] mode (Forward-Weighted CADIS (FW-CADIS) [8] is not currently supported). Shift is coupled to MPACT in VERA for validation and ex-core calculations. Shift uses the information transferred from MPACT for a prior state point to automatically generate a detailed geometric core model for that state point. Shift then samples the fission source provided by MPACT and runs a fixed-source calculation to determine the fluence in the vessel and ex-core features. This fission source can include only the spatial distribution, the full multigroup energy spectrum, or a nuclide-dependent source distribution from MPACT. In the case where only the spatial distribution from MPACT is used, a ${ }^{235} \mathrm{U}$ Watt energy spectrum is used in the Shift calculation. For the case of a nuclide-dependent fission source, Shift samples a Watt energy spectrum based on four nuclides $\left({ }^{235} \mathrm{U},{ }^{238} \mathrm{U},{ }^{239} \mathrm{Pu}\right.$ and $\left.{ }^{241} \mathrm{Pu}\right)$ sampled in a spatial location.

The fixed-source transport can be run in $n$ or $n-\gamma$ mode. For coupled $(n, \gamma)$ problems, the secondary gamma source is sampled from neutron interactions within the core elements during neutron transport. Shift does not currently support prompt fission gammas and delayed fission product gammas as a source in a fixed-source calculation. Also, the current implementation does not have the capability to sample from a user input gamma source. The user should note that the coupled $n-\gamma$ calculation is not optimized and will run significantly slower than neutron-only calculations.

The following sections present the details of running ex-core calculations through VERA. This revision of the manual presents capabilities available in VERA 4.0.1 which uses SCALE version 6.3beta2. Section 2 goes over the input options specific to ex-core calculations with VERA and $\S 3$ explains how to run the ex-core calculations. The output is discussed in $\S 4$ and $\S 5$ gives several examples of vessel fluence and ex-core detector calculations that can be run through VERA. Advanced and experimental capabilities are described in $\S 6$ and a summary of ex-core capabilities in VERA is given in $\S 7$. Finally, Appendix A gives the material template file used for the automated generation of a supplemental ex-core file, Appendix $\mathrm{C}$ gives details on adding and integrating the 
ex-core geometry with VERA, and Appendix D explains the methods and code-coupling algorithms used in VERA for performing these ex-core calculations. 


\section{INPUT}

For VERA ex-core calculations, the Shift parameters are defined in the common VERA input [6] in the SHIFT block. All of the usual inputs for the STATE, CORE, ASSEMBLY, INSERT, CONTROL, DETECTOR, EDITS, MPACT [4], and COBRATF [5] blocks apply to their respective setups. To run any Shift calculation through VERA, the parameter in Listing 1 must be included in the first STATE block. Do not include this extra parameter in subsequent STATE blocks in the same input.

Listing 1. Required STATE parameter.

[STATE]

excore_transport on

! Needed so MPACT knows Shift will run

$\S 2.1$ outlines all of the parameters available in the SHIFT input block. $\S$ B presents the Shift defaults set for ex-core calculations with VERA. Finally, $\S 2.2$ shows a few example SHIFT input blocks for typical ex-core problems.

\subsection{PARAMETERS}

Tables 1 through 4 show the parameters regulating the Shift calculations used in the SHIFT block of the VERA common input. Table 5 gives parameters located in the CORE block of the VERA input used for Shift calculations, and Table 6 gives several experimental parameters. The user should be aware of several caveats when changing some of these advanced parameters.

1. If use_pole_data is true, the other physics broadening parameters do not apply (broaden_xs, delta_t, energy_tol, temperature_tol, union_energy).

2. The core_translate should correspond to the translation used in the supplemental ex-core geometry.

3. When using the supplemental ex-core geometry in CADIS mode, hybrid_tally_names and hybrid_multiplier_names must be specified. The hybrid_tally_names must correspond to tally names in the supplemental ex-core geometry, and only $f l u x$ for hybrid_multiplier_names is supported at this time.

4. num_blocks_i $\times$ num_blocks_j $\mathbf{j}$ should equal the total number of Shift processors.

5. Shift does not model any ex-core geometry (core only) when the problem_mode is set to eigenvalue.

When using the automatic generation of the supplemental ex-core geometry by specifying the bioshield parameter, the user needs to keep in mind the following items:

1. Materials specified in the bioshield and det definitions must exist in the template file located at scripts/Init/omnibus_template.txt (see Appendix A).

2. The generated file will use the base of the VERA input filename and the .omn extension. The user must ensure that once this file is converted to XML, the name matches the filename specified next to the excore_filename parameter in the SHIFT block of the VERA input file. 
3. In CADIS mode, if the VERA input file specifies hybrid_tally_names that do not match a detector ID, the parameter will not be changed. This means the user must know the name of the tally they wish to optimize. If the user-specified tally name does not exist, the calculation is killed. 
Table 1. SHIFT block inputs related to transport and ex-core through VERA.

\begin{tabular}{|c|c|c|c|}
\hline Parameter & Description & Type & Default \\
\hline create_unique_pins & make all pincells unique compositions & bool & false \\
\hline do_transport & perform MC transport & bool & true \\
\hline fiss_src_spectrum & type of spectrum to use for fission source & string in $\{$ "mpact", "nuclide_watt", "u235_watt" \} & "nuclide_watt" \\
\hline lost_particle_error_tolerance & fraction of lost particles to tolerate before aborting & double in $(0,1)$ & $1 e-6$ \\
\hline mode & type of particles to transport & string in $\{" n ", " n p "\}$ & "n" \\
\hline Np & number of particles to transport & double & $1 \mathrm{e} 6$ \\
\hline num_threads & number of threads per core for transport & integer & 1 \\
\hline problem_mode & run mode & string in $\{$ "eigenvalue", "forward", "cadis" $\}$ & "cadis" \\
\hline seed & initialize global random number generator object & integer & 121434 \\
\hline temp_transfer & which temperatures to couple with CTF & string in $\{$ "all", "none", "pin"\} & "all" \\
\hline track_isotopes & which set of isotopes to transfer & string in $\{$ "full", "short" $\}$ & "short" \\
\hline transfer & what to transfer from MPACT & string in \{"all", "fiss_src", "isotopics", "temps" \} & "fiss_src" \\
\hline verbosity & how often to print about particles being transported & string in $\{$ "none", "low", "medium", "high"\} & "low" \\
\hline \multicolumn{4}{|c|}{ Ex-core } \\
\hline bc_bnd_mesh & boundary mesh boundary conditions on all sides & $\begin{array}{l}\text { array of } 6 \text { strings in }\{\text { "reflect", "vacuum" }\}(-X,+X, \\
-Y,+Y,-Z,+Z)\end{array}$ & $\begin{array}{l}\text { \{"vacuum", } \\
\text { "vacuum", } \\
\text { "vacuum", } \\
\text { "vacuum", } \\
\text { "vacuum", } \\
\text { "vacuum"\} }\end{array}$ \\
\hline core_translate & position to translate center of core & array of 3 doubles & $\{0.0,0.0,0.0\}$ \\
\hline excore_filename & $\begin{array}{l}\text { name of Omnibus XML file with ex-core features and } \\
\text { tallies }\end{array}$ & string & none \\
\hline hybrid_multiplier_names & ex-core tally multipliers to optimize for CADIS & array of strings & none \\
\hline hybrid_tally_names & ex-core tally names to optimize for CADIS & array of strings & none \\
\hline raytrace_resolution & resolution for geometry ray trace & integer & 1024 \\
\hline vera_pressure_vessel & pull in outer pressure vessel radius from VERA input & bool & false \\
\hline x_bnd_mesh & boundary mesh $x$-axis limits & array of 2 doubles & none \\
\hline y_bnd_mesh & boundary mesh $y$-axis limits & array of 2 doubles & none \\
\hline z_bnd_mesh & boundary mesh $z$-axis limits & array of 2 doubles & none \\
\hline
\end{tabular}


Table 2. SHIFT block inputs related to hybrid MC/deterministic through VERA.

\begin{tabular}{|c|c|c|c|}
\hline Parameter & Description & Type & Default \\
\hline \multicolumn{4}{|c|}{ Hybrid MC/Deterministic } \\
\hline adjoint & perform adjoint solve & bool & true \\
\hline azimuthals_octant & number of azimuthal angles per octant for $S_{N}$ & positive integer & 4 \\
\hline cell_homogenize & homogenize material in cells & bool & true \\
\hline downscatter & downscatter-only & bool & false \\
\hline eq_set & solution method or spatial discretization & $\begin{array}{l}\text { string in }\{\text { "bld", "bld_2d", "ld", } \\
\text { "sc" }\}\end{array}$ & "sc" \\
\hline extend_axial_mesh_size & adjoint axial mesh size in ex-core region & double & 5.0 \\
\hline grp_collapse_src & source to do group collapse & array of doubles & depends on xs_library \\
\hline iterate_downscatter & iterate over downscatter groups & bool & false \\
\hline max_delta_z & maximum core mesh size in $\mathrm{z}$ & double & 5.0 \\
\hline mesh & number of mesh cells per pincell & positive integer & 1 \\
\hline new_grp_bounds & collapsed group lower boundaries & array of decreasing doubles & $\begin{array}{lll}\{6.0653 \mathrm{e} 6, & 3.6788 \mathrm{e} 6, & 2.3457 \mathrm{e} 6, \\
1.6530 \mathrm{e} 6, \quad 8.2085 \mathrm{e} 5, & 2.4176 \mathrm{e} 4, \\
1.0130 \mathrm{e} 2,1.0000 \mathrm{e}-5\} & \end{array}$ \\
\hline num_blocks_i & number of partitions (processors) in $\mathrm{x}$ & positive integer & 10 \\
\hline num_blocks_j & number of partitions (processors) in $y$ & positive integer & 10 \\
\hline num_groups & number of energy groups & positive integer & 8 \\
\hline num_sets & number of energy sets & positive integer & 1 \\
\hline num_z_blocks & number of pipelining blocks in $\mathrm{z}$ & positive integer & depends on number of processors \\
\hline output_adjoint & $\begin{array}{l}\text { output adjoint flux and pin adjoint to Shift HDF5 file } \\
\text { and adjoint source to separate HDF5 file }\end{array}$ & bool & false \\
\hline partition_upscatter & partition energy over upscatter groups only & bool & false \\
\hline pin_partitioning & partition mesh over pincells & bool & false \\
\hline Pn_correction & $\begin{array}{l}\text { use outscatter-corrected diffusion coefficient to reduce } \\
\text { memory in solve }\end{array}$ & bool & false \\
\hline Pn_order & order of moments & integer & 0 \\
\hline polars_octant & number of polar angles per octant for $S_{N}$ & positive integer & 2 \\
\hline quad_type & type of $S_{N}$ quadrature & $\begin{array}{l}\text { string in \{"galerkin", "glproduct", } \\
\text { "ldfe", "levelsym", "qr"\} }\end{array}$ & "qr" \\
\hline
\end{tabular}


Table 3. SHIFT block inputs related to hybrid MC/deterministic

(continued) and output through VERA.

\begin{tabular}{|c|c|c|c|}
\hline Parameter & Description & Type & Default \\
\hline \multicolumn{4}{|c|}{ Hybrid MC/Deterministic } \\
\hline refl_mesh_size & radial mesh size in ex-core region & double & 5.0 \\
\hline src_disc_12_error & maximum L2 error for point-sampling discretization & double in $(0,1)$ & $1 e-3$ \\
\hline src_disc_max_samples & maximum number of discretization samples & integer & $1 \mathrm{e} 9$ \\
\hline src_disc_samples_per_batch & number of samples per point-sampling batch & integer & $1 \mathrm{e} 7$ \\
\hline store_fulcrum_string & save fulcrum string as file & bool & false \\
\hline upscatter_max_itr & maximum number of iterations for deterministic solve & integer & $1 \mathrm{e} 3$ \\
\hline upscatter_solver & upscatter solver & string in $\{$ "gauss_seidel", "gmres" $\}$ & "gauss_seidel" \\
\hline upscatter_subspace_size & upscatter solver subspace size & integer & 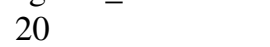 \\
\hline upscatter_tolerance & upscatter solver convergence tolerance & double & $1 \mathrm{e}-4$ \\
\hline upscatter_verbosity & log verbosity for upscatter solver & string in \{"none", "low", "medium", "high"\} & "high" \\
\hline within_group_max_itr & maximum number of iterations for within group solver & integer & $1 \mathrm{e} 3$ \\
\hline within_group_solver & within-group solver & string in $\{$ "gmres" $\}$ & "gmres" \\
\hline within_group_subspace_size & max subspace size for within group solver & integer & 20 \\
\hline within_group_tolerance & within group solver convergence tolerance & double in $(0,1)$ & $1 \mathrm{e}-4$ \\
\hline within_group_verbosity & log verbosity for within group solver & string in $\{$ "none", "low", "medium", "high"\} & "low" \\
\hline ww_decomp & type of adjoint flux decomposition & string in $\{$ "full", "separable" $\}$ & "separable" \\
\hline xs_library & name of SCALE MG data library file & string & "v7-56" \\
\hline \multicolumn{4}{|c|}{ Output } \\
\hline do_output & do Shift output & bool & true \\
\hline local_log & level of local node log information level & $\begin{array}{l}\text { string in \{ "debug", "diagnostic", "status", } \\
\text { "info", "warning", "error", "critical"\} }\end{array}$ & "error" \\
\hline global_log & level of global log information & $\begin{array}{l}\text { string in }\{\text { "debug", "diagnostic", "status", } \\
\text { "info", "warning", "error", "critical" \} }\end{array}$ & "info" \\
\hline ray trace_levels & $\mathrm{z}$ levels to ray trace geometry and output & array of doubles & $\begin{array}{l}\text { midpoint of active } \\
\text { fuel or core axial } \\
\text { levels }\end{array}$ \\
\hline rtk_output_format & format of core RTK geometry output file & string in $\{$ "xml", "hdf5" $\}$ & "hdf5" \\
\hline output_fission_source & output the initial fission source for each state & bool & false \\
\hline output_geometry & $\begin{array}{l}\text { output HDF5 files of ray traced geometry (initial) and composi- } \\
\text { tions (each state) }\end{array}$ & bool & true \\
\hline output_micro_tally & output micro reaction tallies & bool & false \\
\hline output_ww & output the weight windows if used & bool & false \\
\hline
\end{tabular}


Table 4. SHIFT block inputs related to eigenvalue, physics, and tallies through VERA.

\begin{tabular}{|c|c|c|c|}
\hline Parameter & Description & Type & Default \\
\hline \multicolumn{4}{|c|}{ Physics Treatment } \\
\hline broaden_xs & apply cross-section doppler broadening & bool & true \\
\hline ce_lib_path & path to alternate SCALE CE data library file not in VERAData & string & none \\
\hline dbrc & apply doppler-broadening resonance correction & bool & false \\
\hline delta_t & finite difference grid spacing for Leal-Hwang interpolation of cross sections & positive double & 1.0 \\
\hline energy_tol & relative difference for considering two energy points equal & double in $(0,1)$ & $1 \mathrm{e}-10$ \\
\hline temperature_tol & tolerance for reusing existing broadened cross sections & positive double & 4.0 \\
\hline thermal_energy_cutoff & cutoff for treatment of thermal neutrons & double & $10.0 \mathrm{eV}$ \\
\hline union_energy & unionize lower and upper library temperature energy grids & bool & true \\
\hline use_pole_data & use pole CE data for on-the-fly doppler broadening & bool & false \\
\hline \multicolumn{4}{|c|}{ Tallies } \\
\hline do_micro_tally & tally microscopic reaction rate in eigenvalue mode & bool & false \\
\hline gamma_flux & tally the photon flux in each pincell & bool & false \\
\hline micro_zaids & nuclides to tally micro reactions in eigenvalue mode & array of integers & $\{92235,92238\}$ \\
\hline micro_rxns & MT of micro reactions to tally in eigenvalue mode & array of integers & $\{18,102\}$ \\
\hline radial_mesh & radii for flux tally in vessel & array of doubles & vessel radii \\
\hline n_bounds & neutron energy bounds for tallies & array of decreasing doubles & none \\
\hline num_axial & number of axial levels for flux tallies in vessel & integer & 1 \\
\hline num_theta & number of theta divisions for flux tallies in vessel in $[0,2 \pi]$ & integer & 1 \\
\hline p_bounds & photon energy bounds for tallies & array of decreasing doubles & none \\
\hline \multicolumn{4}{|c|}{ Eigenvalue Only } \\
\hline num_cycles & total number of eigenvalue cycles & integer & 50 \\
\hline num_inactive_cycles & number of inactive eigenvalue cycles & integer & 10 \\
\hline
\end{tabular}


Table 5. Shift-related input parameters in the CORE block.

\begin{tabular}{|c|c|c|c|}
\hline Parameter & Description & Type & Default \\
\hline bioshield & bioshield materials and radii & array of alternating strings and doubles & none \\
\hline det & detector id, type, radii, materials, heights, response type, and well type & strings and doubles; & none \\
\hline det locations & detector id, radius, degree, and elevation & $\begin{array}{l}\text { type: string in }\{\text { "PWR", "SRC" }\} \text {, } \\
\text { response type : string in }\{\text { "b10", "u2 } 235 "\} \text {, } \\
\text { well type: string in }\{\text { "none", "wedge" }\} \\
\text { string and double }\end{array}$ & none \\
\hline det_locations & detector id, radius, degree, and elevation & string and double & none \\
\hline
\end{tabular}

Table 6. Experimental temperature homogenization SHIFT block input parameters.

\begin{tabular}{|c|c|c|c|}
\hline Parameter & Description & Type & Default \\
\hline homog_type & if homogenizing, go by assembly or in rings & string in $\{$ "assem", "rings" $\}$ & none \\
\hline homog_explicit_ring & radius to homogenize within and have explicit pins outside of & positive double & none \\
\hline homog_pin_rings & homogenize according to pin locations, instead of assembly locations & bool & false \\
\hline homog_ring_radii & radii for homogenization, if homog_type is rings & array of increasing doubles & none \\
\hline
\end{tabular}




\subsection{EXAMPLE SHIFT INPUT BLOCKS}

An example of the SHIFT block in the VERA input with a default vessel tally is shown in Listing 2. This example would run an analog Shift calculation using $1 \times 10^{7}$ particle histories with the specified vessel fluence tally and the other default parameters listed in Listing B.

Another example of the SHIFT block that includes modified parameters for a CADIS run is shown in Listing 3. The modified $S_{N}$ parameters control the deterministic adjoint solve. This example would run a CADIS Shift calculation on 25 processors with the specified vessel fluence tally. The adjoint calculation would use the collapsed group structure (collapsed from the default 56 group SCALE library).

Finally, an example of the SHIFT block using a supplemental ex-core geometry file and associated input parameters is shown in Listing 4. The details of the ex-core geometry and tallies are defined in a supplemental ex-core file (not shown here). This input would perform a CADIS Shift calculation to optimize the flux reaching several detectors and produce a detector response. A few things to note: first, the full pressure vessel is being pulled in from the VERA input to combine with a supplemental ex-core file geometry; second, the core is translated to correspond with the origin used by the supplemental ex-core geometry. Finally, the boundary mesh given is for quarter core symmetry and is input based on the supplemental geometry origin. More details on setting up and using a supplemental ex-core geometry file are given in $\S 5$ and $\S \mathrm{C}$.

Section 5 presents full example VERA inputs for several different ex-core calculations. This includes an example of using the automated detector and bioshield setup. For further details on input, running, and output of the experimental homogenization methods (with inputs given in Table 6), please contact Tara Pandya (pandyatm@ornl . gov) at Oak Ridge National Laboratory (ORNL).

\section{Listing 2. Example SHIFT block with vessel tally in VERA common input.}

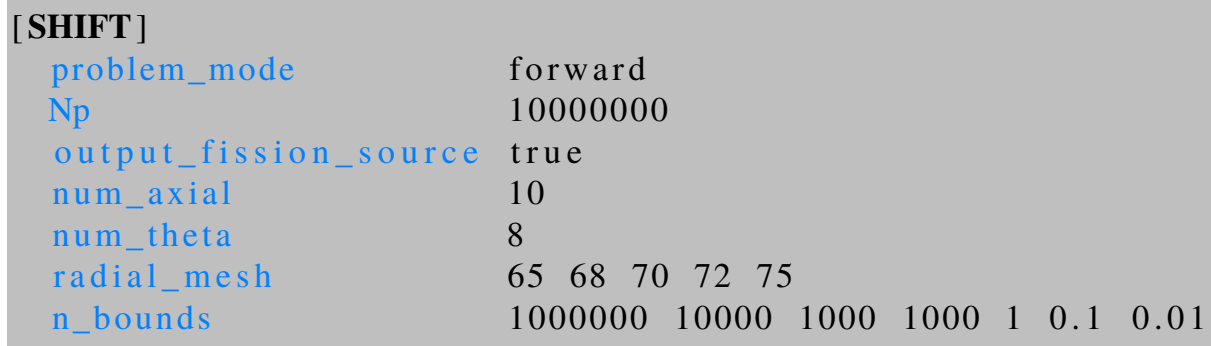


Listing 3. Example SHIFT block with hybrid parameters and vessel tally in VERA common input.

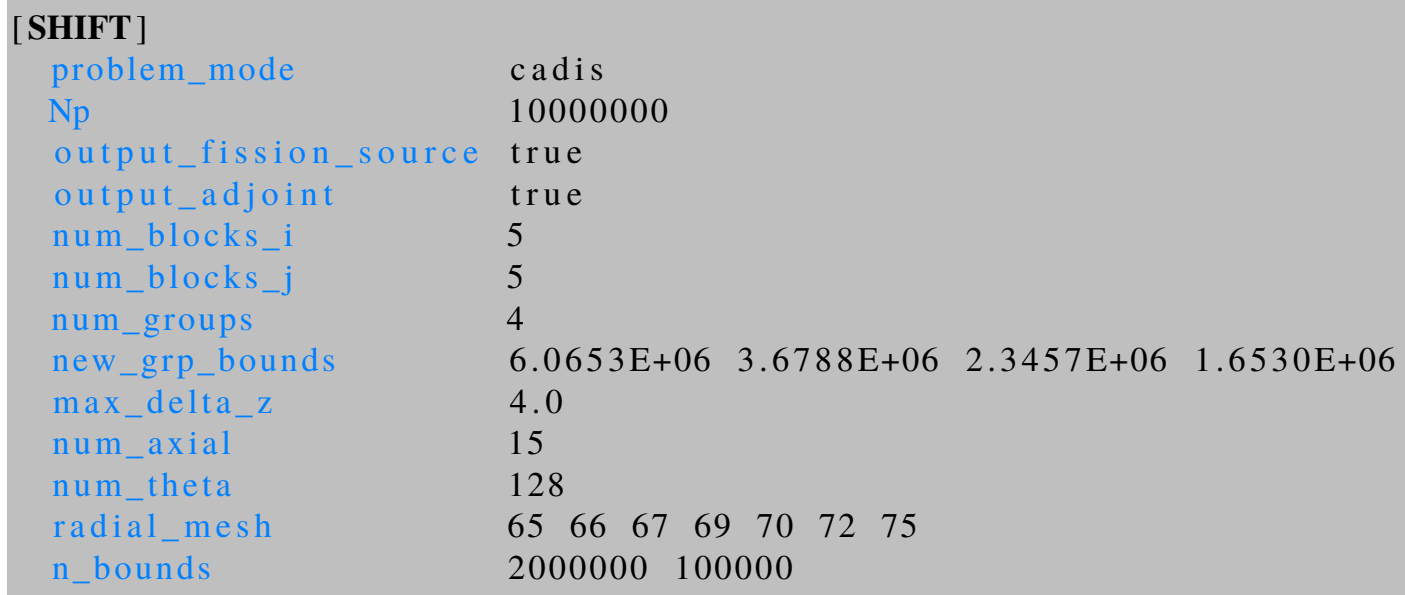

Listing 4. Example SHIFT block with ex-core parameters in VERA common input.

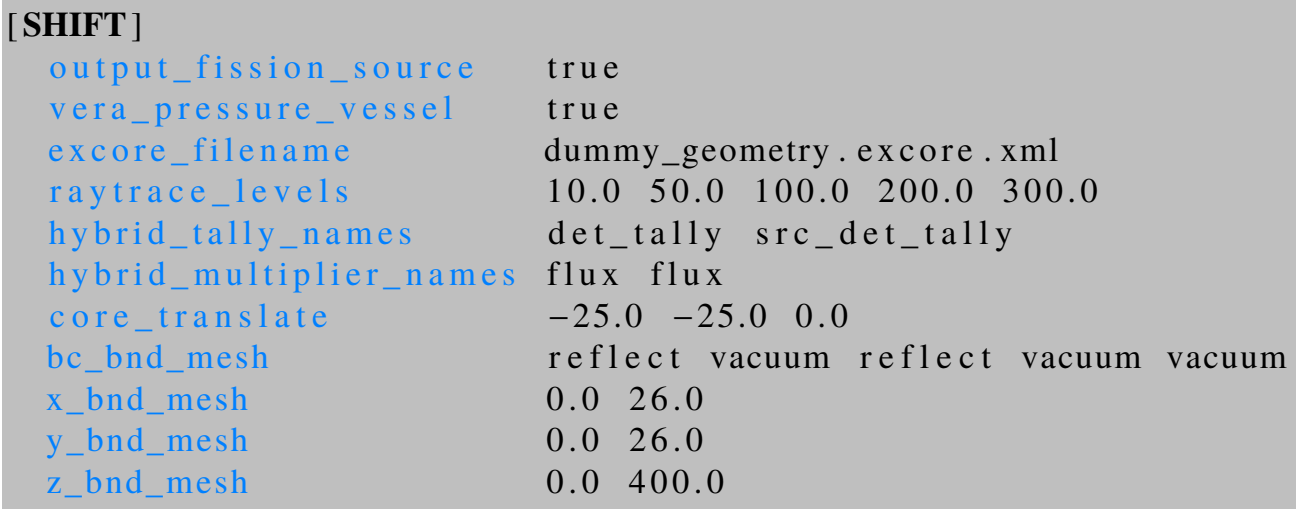




\section{RUNNING}

There are two ways to run ex-core calculations with VERA: with verarun and manually. Note that since MPACT and Shift run on their own sets of processors, at least two processors are always required to run ex-core calculations. When running a CADIS calculation, the number of processors that Shift can run on is limited by the decomposition chosen for the adjoint deterministic calculation (as mentioned in $\S 2.1$ ).

\subsection{RUNNING WITH VERARUN}

The simplest way to run an ex-core calculation is to use the verarun executable, assuming it is installed on the machine. If the VERA environment is set up on the machine, a simple run command using a VERA input file called sample.inp can be used, such as the following example.

\section{verarun sample.inp}

This command automates all of the file generation (detailed in § 3.2) and submits a job using whatever job scheduler is used by the machine (portal batch system (PBS) or Slurm). If running an eigenvalue or forward-mode calculation, Shift will only run on one processor unless the nprocs parameter is given in a RUN block in the VERA input to specify the total number of processors. If the nprocs parameter is given, Shift will use the leftover processors that remain after the requested number for MPACT is fulfilled. The MPACT VERA user input manual provides details on the MPACT parallel decomposition [4].

The verarun executable can also be run without automatically submitting your job to allow for editing the submission script, allowing customization of the processor layout and other advanced options. This command is the following:

\section{verarun $-x$ sample.inp}

Listing 5 shows the relevant parameters in the VERA input to specify a CADIS calculation with 32 processors for MPACT and 16 processors for Shift. Another simple example showing the relevant parameters for running a Shift forward calculation with 32 processors for MPACT and 32 processors for Shift is given in Listing 6.

\section{Listing 5. Example processor specification for CADIS ex-core calculation using 48 total processors.}

$\begin{array}{ll}\text { [MPACT] } & \\ \text { num_space } & 32 \\ & \\ \text { [SHIFT ] } & \\ \text { problem_mode } & \text { cadis } \\ \text { num_blocks_i } & 4 \\ \text { num_blocks_j } & 4\end{array}$




\section{Listing 6. Example processor specification for forward ex-core calculation with verarun on 64 total processors.}

[MPACT $]$

num_space 32

[SHIFT ]

problem_mode forward

[RUN]

nprocs $\quad 64$

\subsection{RUNNING MANUALLY}

All of the automation encapsulated in verarun can also be performed separately using the vera_to_shift executable. If using a supplemental ex-core file, the ASCII input must be converted to XML before running vera_to_shift. The step-by-step process for running an ex-core calculation is as follows:

1. Run the react $2 x m l$ Perl script to convert the VERA input file to XML.

2. If using a supplementary ex-core geometry file, run excore $2 x m l$ on the Omnibus input file.

3. Run vera_to_shift.

For example, for the VERA input file sample.vera and supplemental ex-core geometry file sample.excore.omn, the following series of commands will convert the inputs to XML and run the problem on 16 processors.

\section{./ react 2 xml sample.vera}

\section{./ excore $2 x m l$ sample.excore.omn -o sample.excore.xml}

\section{mpirun -np 16 ./ vera_to_shift --case=sample}

Finally, the ability to run Shift without coupling to MPACT using the VERA common input also exists. This executable will run a Shift eigenvalue calculation of the given single statepoint VERA input and produces $k_{\text {eff }}$ and pin powers written to the Shift HDF5 output file. Using the same preceding example, the first two commands would be the same to convert the inputs to XML. To run the problem on 16 processors the command is the following.

\section{mpirun $-\mathrm{np} 16$./ vera_shift - i=sample.xml}




\section{OUTPUT}

From an ex-core calculation, the relevant output quantities are located in different output files depending on the type of ex-core calculation performed. The usual VERA HDF5 output file is produced along with the associated output files from MPACT and CTF. This output file can include some results from the Shift calculation, while the majority of the Shift output is found in the separate *.shift.h5 output file.

The following section presents an overview of the data available in output files and where the user can expect specific data to be located based on the type of calculation. Upon completion of the ex-core calculation, the user can postprocess and plot the tallies and other quantities of interest over all states from the output files. This section presents the plotting capability in VERAView [9]. Details on some of the advanced postprocessing that can be performed on the output can be found in $\S 6$.

\subsection{GENERAL OUTPUT}

Regardless of the type of ex-core calculation, the Shift HDF5 output file contains general output information. Listings 7 and 8 gives an example of output data produced from most calculations. This output was produced from a problem with a single state point where the fission source was output. The CORE and metadata groups are always present. A STATE_\#\#\#\# group exists for each state point specified in the VERA common input. Each state point group contains the appropriate fission source groups and datasets if the user-specified output_fission_source is set to true.

If the adjoint is being calculated, the user can also choose to have the adjoint flux, pin adjoint, and adjoint source written with the output_adjoint parameter. The adjoint flux and pin adjoint are written to the first state point, since they are currently only calculated for the first state in a cycle, as shown in Listing 7. The adjoint source is written to an independent file named adjoint_source.h5. The details of each adjoint flux data field in the Shift HDF5 file adjoint group are described in Table 7. The details of each fission source data field in the HDF5 file fission_source group are described in Table 8.

If the geometry output is turned on (output_geometry set to true), composition output files are created corresponding to each state point (*.shift_state_\#_compositions.h5), as well as a ray trace file (*.shift_state_1_ray trace.h5). Note that a ray trace file is only created for the first state point. The user can specify the levels to perform the ray trace with the ray trace_levels parameter; if no levels are specified, the core midplane is used. These files can be used for advanced geometry visualization with Python as discussed in $\S 6$.

\subsection{EIGENVALUE CALCULATION}

From an eigenvalue calculation, results such as Shannon entropy, $k_{\text {eff }}$, active cycles, and pin powers, are located in the group for each state point in the Shift HDF5 output file. An example of the structure of eigenvalue-specific results in the output file is given in Listing 9 for a problem with multiple state points. 


\section{Listing 7. General data in Shift HDF5 output file. File contents generated via h5dump $-\mathrm{n}$.}

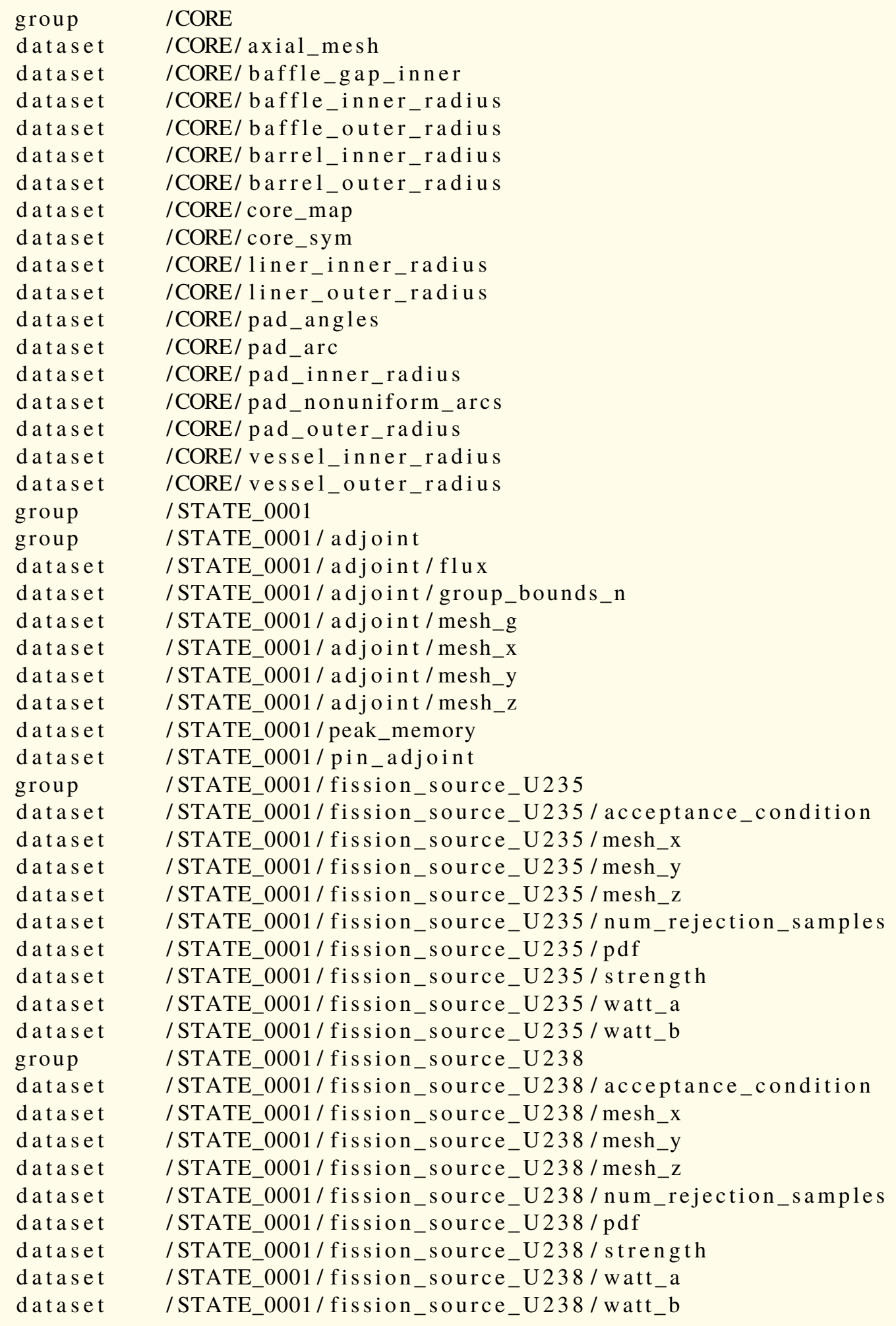


Listing 8. General data in Shift HDF5 output file (continued).

$\begin{array}{ll}\text { group } & \text { / metadata } \\ \text { dataset } & \text { / metadata/datetime } \\ \text { dataset } & \text { / metadata/scale_rev } \\ \text { dataset } & \text { / metadata/scale_version } \\ \text { dataset } & \text { /metadata/system } \\ \text { dataset } & \text { / veraout_version }\end{array}$

Table 7. Adjoint flux data fields in the Shift HDF5 output file.

\begin{tabular}{lll}
\hline Data name & Type & Description \\
\hline flux & array of doubles & energy-dependent adjoint flux $[\mathrm{g}][\mathrm{z}][\mathrm{y}][\mathrm{x}]$ \\
group_bounds_n & array of doubles & energy group boundaries in decreasing order $[\mathrm{eV}]$ \\
mesh_g & array of integers & group indices \\
mesh_x & array of doubles & adjoint flux mesh edges along x-axis \\
mesh_y & array of doubles & adjoint flux mesh edges along y-axis \\
mesh_z & array of doubles & adjoint flux mesh edges along z-axis \\
\hline
\end{tabular}

Table 8. Fission source data fields in the HDF5 output file.

\begin{tabular}{lll}
\hline Data name & Type & Description \\
\hline mesh_x & array of doubles & fission source mesh edges along x-axis \\
mesh_y & array of doubles & fission source mesh edges along y-axis \\
mesh_z & array of doubles & fission source mesh edges along z-axis \\
mesh_g $g^{\dagger}$ & array of doubles & fission source energy group bounds \\
pdf & array of doubles & fission source strength ${ }^{*}[\mathrm{z}][\mathrm{y}][\mathrm{x}]$ or $^{\dagger}[\mathrm{g}][\mathrm{z}][\mathrm{y}][\mathrm{x}]$ \\
strength & scalar & fission source strength \\
watt_a* & scalar & Watt spectrum constant $(\mathrm{MeV})$ \\
watt_b ${ }^{*}$ & scalar & Watt spectrum constant $(1 / \mathrm{MeV})$ \\
\hline
\end{tabular}

$\dagger$ If the fiss_src_spectrum type is mpact

*If the fiss_src_spectrum type is nuclide_watt or $u 235 \_w a t t$ 
Listing 9. Data in Shift HDF5 output file from an eigenvalue calculation. File contents generated via h5dump $-n$.

\begin{tabular}{|c|c|}
\hline group & /STATE_0001 \\
\hline dataset & /STATE_0001/active_cycles \\
\hline dataset & /STATE_0001/ entropy \\
\hline dataset & /STATE_0001/entropy_active_deviance \\
\hline dataset & /STATE_0001/entropy_check \\
\hline dataset & /STATE_0001/entropy_crossover_cycle \\
\hline dataset & /STATE_0001/first_active_cycle \\
\hline dataset & /STATE_0001/ keff \\
\hline dataset & /STATE_0001/keff_sigma \\
\hline dataset & /STATE_0001/peak_memory \\
\hline dataset & /STATE_0001/pin_powers \\
\hline dataset & /STATE_0001/pin_powers_sigma \\
\hline dataset & /STATE_0001/total_cycles \\
\hline group & /STATE_0002 \\
\hline dataset & /STATE_0002/active_cycles \\
\hline dataset & /STATE_0002/ entropy \\
\hline dataset & /STATE_0002/entropy_active_deviance \\
\hline dataset & /STATE_0002/entropy_check \\
\hline dataset & /STATE_0002/entropy_crossover_cycle \\
\hline dataset & /STATE_0002/first_active_cycle \\
\hline dataset & /STATE_0002/ keff \\
\hline dataset & /STATE_0002/keff_sigma \\
\hline dataset & /STATE_0002/peak_memory \\
\hline dataset & /STATE_0002/pin_powers \\
\hline dataset & /STATE_0002/pin_powers_sigma \\
\hline dataset & /STATE_0002/total_cycles \\
\hline
\end{tabular}




\subsection{SIMPLE VESSEL FLUENCE CALCULATION}

From a simple vessel fluence calculation in which only the VERA common input file is used (i.e., there is no supplemental ex-core geometry file), the vessel flux is automatically tallied even if the user does not specify the tally parameters in the SHIFT block. The default vessel flux tally has a radial bin for each radius given in the vessel card, one axial bin, one theta bin, and one energy bin. The vessel flux tally result is automatically passed from Shift to VERA at each state point and is output to both the VERA and Shift HDF5 output files. An example of the Shift output for two state points is shown in Listing 10. The details of each tally data field in the HDF5 file vessel_tally group are described in Table 9. Note that there can also be photon boundaries specified for the vessel tally if running an ex-core calculation with $\operatorname{Shift}$ in $(n, \gamma)$ mode.

Table 9. Vessel tally data fields in the Shift HDF5 output file. (All data fields are stored in the /STATE_nnnn/tally/vessel_tally group.)

\begin{tabular}{|c|c|c|}
\hline Data name & Type & Description \\
\hline binned & array of doubles & $\begin{array}{l}\text { energy-binned vessel flux mean and variance } \\
{[\mathrm{g}][\mathrm{z}][\text { theta][r][multiplier][stat] }}\end{array}$ \\
\hline description & string & description of tally \\
\hline group_bounds_n & array of doubles & energy bin boundaries in decreasing order $(\mathrm{eV})$ \\
\hline \multicolumn{2}{|c|}{ max_encountered_bins int } & $\begin{array}{l}\max \text { number of tally bins encountered by one particle } \\
\text { history }\end{array}$ \\
\hline esh_r & array & tally mesh radii \\
\hline & arra & mean \\
\hline sh_theta & f doubles & tally mesh azimuthal angles around full core \\
\hline$s h \_z$ & array of doubles & tally mesh axial edges \\
\hline multiplier_descs & array of strings & description of flux \\
\hline multiplier_names & array of strings & flux \\
\hline normalization & double & normalization factor \\
\hline num_histories & double & number of particle histories tallied \\
\hline total & array of doubles & $\begin{array}{l}\text { total neutron and photon vessel flux mean and vari- } \\
\text { ance }[\mathrm{z}][\text { theta][r][multiplier][stat] }\end{array}$ \\
\hline & & Cartesian translation of mesh $[\mathrm{x}][\mathrm{y}][\mathrm{z}]$ \\
\hline & arrs & volumes of tally mesh [z][theta][r] \\
\hline
\end{tabular}

\subsection{DETECTOR RESPONSE CALCULATION}

For a detector response calculation, a typical use case is to use a Shift cell tally in the detector regions of interest. This can be done automatically with the detector and bioshield inputs from the VERA common input or with the supplemental ex-core file as mentioned previously. Table 10 gives the detail of a detector cell tally output in the Shift HDF5 output file. Again, note that photon energy bounds could also be present if the Shift calculation was run in the $(n, \gamma)$ mode. 
Listing 10. Data in Shift HDF5 output file from a vessel fluence calculation. File output was generated via $h 5$ dump $-n$

\begin{tabular}{|c|c|}
\hline group & /STATE_0001 \\
\hline group & /STATE_0001/ t a $11 \mathrm{y}$ \\
\hline group & /STATE_0001/tally/vessel_tally \\
\hline dataset & /STATE_0001/tally/vessel_tally/binned \\
\hline dataset & /STATE_0001/tally/vessel_tally/description \\
\hline dataset & /STATE_0001/tally/vessel_tally/group_bounds_n \\
\hline dataset & /STATE_0001/tally/vessel_tally/max_encountered_bins \\
\hline dataset & /STATE_0001/tally/vessel_tally/mesh_r \\
\hline dataset & /STATE_0001/tally/vessel_tally/mesh_stat \\
\hline dataset & /STATE_0001/tally/vessel_tally/mesh_theta \\
\hline dataset & /STATE_0001/tally/vessel_tally/mesh_z \\
\hline dataset & /STATE_0001/tally/vessel_tally/multiplier_descs \\
\hline dataset & /STATE_0001/tally/vessel_tally/multiplier_names \\
\hline dataset & /STATE_0001/tally/vessel_tally/normalization \\
\hline dataset & /STATE_0001/tally/vessel_tally/num_histories \\
\hline dataset & /STATE_0001/tally/vessel_tally/total \\
\hline dataset & /STATE_0001/tally/vessel_tally/translation \\
\hline dataset & /STATE_0001/tally/vessel_tally/volumes \\
\hline group & /STATE_0002 \\
\hline group & /STATE_0002/ tally \\
\hline group & /STATE_0002/tally/vessel_tally \\
\hline dataset & /STATE_0002/tally/vessel_tally/binned \\
\hline dataset & /STATE_0002/tally/vessel_tally/description \\
\hline dataset & /STATE_0002/tally/vessel_tally/group_bounds_n \\
\hline dataset & /STATE_0002/tally/vessel_tally/max_encountered_bins \\
\hline dataset & /STATE_0002/tally/vessel_tally/mesh_r \\
\hline dataset & /STATE_0002/tally/vessel_tally/mesh_stat \\
\hline dataset & /STATE_0002/tally/vessel_tally/mesh_theta \\
\hline dataset & /STATE_0002/tally/vessel_tally/mesh_z \\
\hline dataset & /STATE_0002/tally/vessel_tally/multiplier_descs \\
\hline dataset & /STATE_0002/tally/vessel_tally/multiplier_names \\
\hline dataset & /STATE_0002/tally/vessel_tally/normalization \\
\hline dataset & /STATE_0002/tally/vessel_tally/num_histories \\
\hline dataset & /STATE_0002/tally/vessel_tally/total \\
\hline dataset & /STATE_0002/tally/vessel_tally/translation \\
\hline dataset & /STATE_0002/tally/vessel_tally/volumes \\
\hline
\end{tabular}


Table 10. Cell tally data fields in the Shift HDF5 output file. (All cell tallies are stored in the /STATE_nnnn/tally group.)

\begin{tabular}{|c|c|c|}
\hline Data name & Type & Description \\
\hline binned & array of doubles & $\begin{array}{l}\text { energy-binned flux mean and variance } \\
{[\mathrm{g}][\text { cell][multiplier] }[\text { stat }]}\end{array}$ \\
\hline description & string & description of tally \\
\hline group_bounds_n & array of doubles & energy bin boundaries in decreasing order $(\mathrm{eV})$ \\
\hline \multicolumn{2}{|c|}{ max_encountered_bins int } & $\begin{array}{l}\text { max number of tally bins encountered by one particle } \\
\text { history }\end{array}$ \\
\hline mesh_stat & array of strings & mean and var \\
\hline multiplier_descs & array of strings & description of each response tallied \\
\hline multiplier_names & array of strings & scalars and responses tallied \\
\hline normalization & double & normalization factor \\
\hline num_histories & double & number of particle histories tallied \\
\hline total & array of doubles & $\begin{array}{l}\text { total neutron and photon flux mean and variance } \\
\text { [cell][multiplier][stat] }\end{array}$ \\
\hline union_cellids & array of ints & internal cell indices in this tally \\
\hline union_lengths & array of ints & number of cell unions making up this tally \\
\hline volumes & array of doubles & volumes of tally cells \\
\hline
\end{tabular}

\subsection{VISUALIZATION WITH VERAVIEW}

As mentioned previously, vessel flux calculated by Shift is reported back to MPACT for each state point to accumulate fluence. Fluence, $\phi(\vec{r}, E)$, is defined as the time integration of the flux, $\phi(\vec{r}, E, t)$, as given by

$$
\phi(\vec{r}, E)=\int \phi(\vec{r}, E, t) \mathrm{d} t
$$

In practice, the fluence, $\phi_{M}^{g}(\vec{r})$, at a specific time step becomes

$$
\phi_{M}^{g}(\vec{r})=\sum_{m=1}^{M} \frac{\Delta t_{m}}{2}\left(\phi_{m-1}^{g}(\vec{r})+\phi_{m}^{g}(\vec{r})\right),
$$

where $M$ is the current time step and $g$ is the energy group. The flux, $\phi_{m}^{g}(\vec{r})$, in Eq. (2) comes from the energy-binned vessel tally for each state passed from Shift to MPACT. The end time, $t_{m}$, for a given state is calculated using the effective full-power days (EFPD) (aka exposure) stored in MPACT converted to seconds:

$$
t_{m}=86400 \times \mathrm{EFPD}_{m} .
$$

Specifically, MPACT performs numerical integration via the trapezoidal rule to accumulate the fluence. MPACT also propagates the associated MC uncertainty on the fluence, and this is output to the VERA HDF5 file as well.

The vessel fluence output to the MPACT HDF5 file can be visualized and analyzed in VERAView along with other core parameters such as pin powers. Figures 1-2 show examples of plotting the 
vessel flux, vessel fluence, and pin powers in VERAView. These two figures show the location of the maximum vessel flux and maximum vessel fluence. VERAView can also show the pin-resolved adjoint from the first state (which is currently the only state that performs the adjoint calculation) that is output to the Shift HDF5 file, as shown in Fig. 3. 


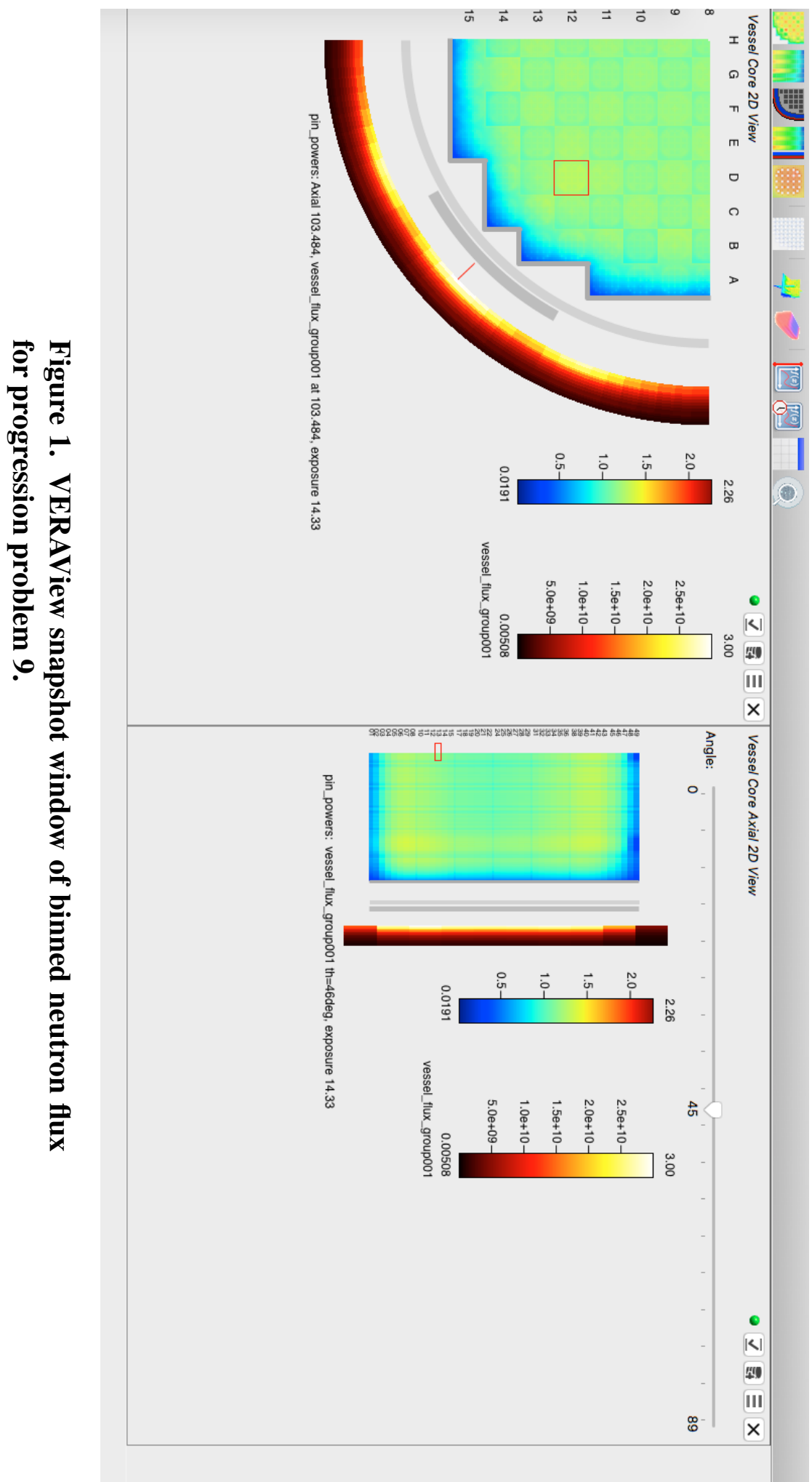




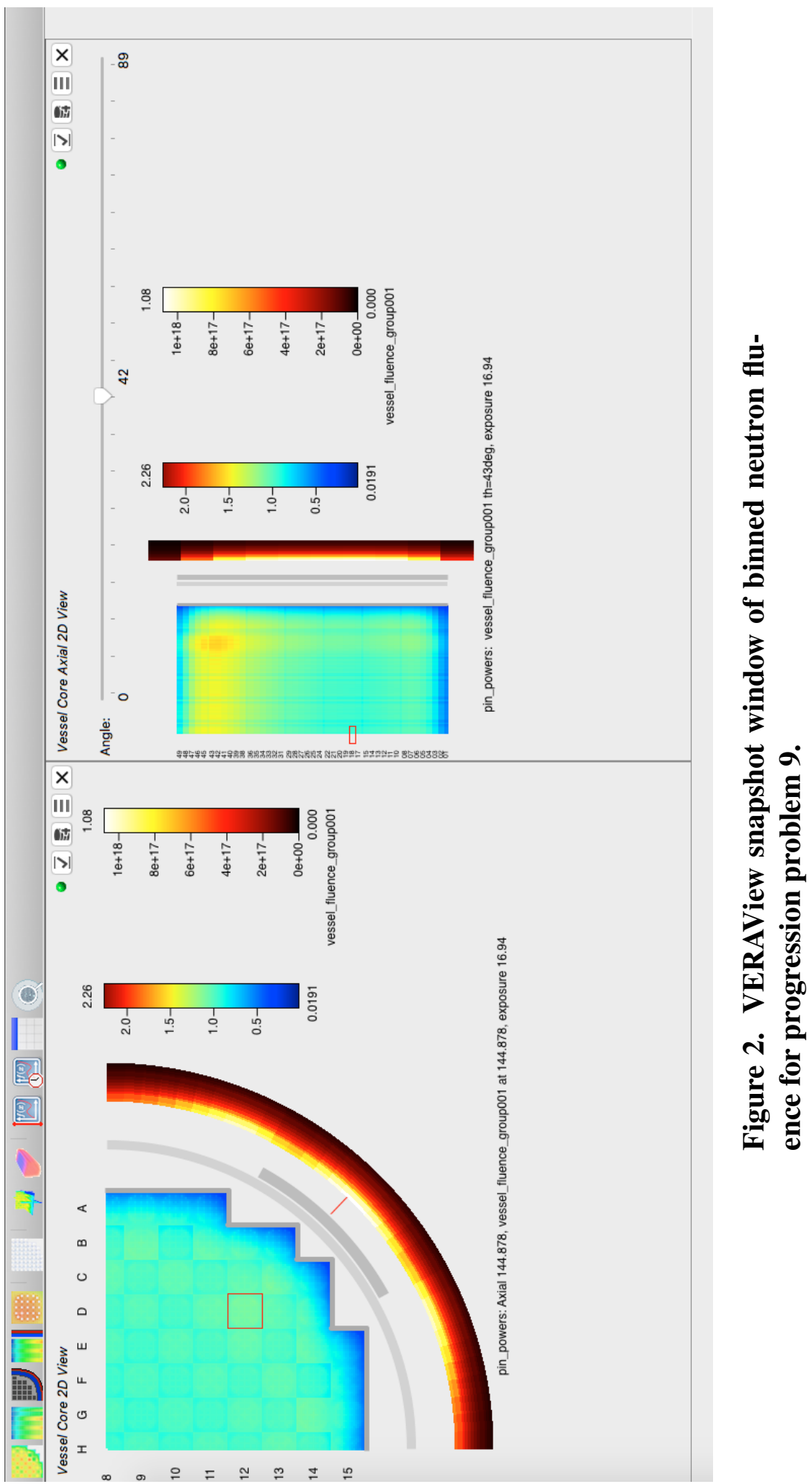




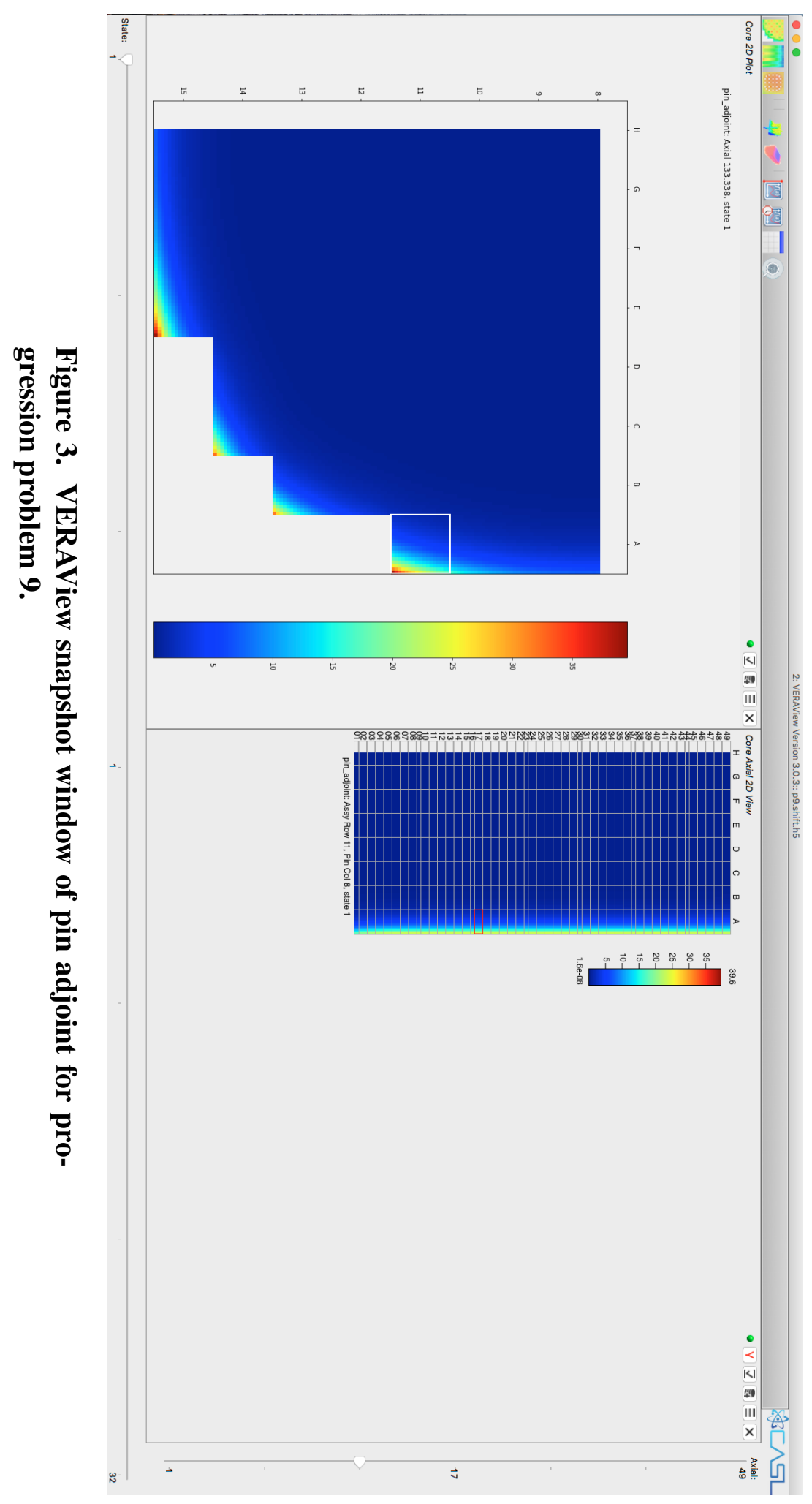




\section{EXAMPLES}

This section presents inputs for several types of ex-core problems that can be run with VERA. Many variations and input parameters can be used for ex-core calculations; the following examples should be used as guidance for how a typical user might set up a problem.

\subsection{SHIFT EIGENVALUE ASSEMBLY EXAMPLE}

This first example input is a modified version of CASL progression problem 3a [10]. The VERA common input for this problem is publicly available on Github [11]. It serves to show the input mechanics for running Shift in eigenvalue mode through VERA. Listing 11 shows the full VERA input for this example, and a ray trace through the geometry axial midplane is shown in Fig. 4. The SHIFT block is set up to perform an eigenvalue calculation with micro reaction rate tallies in the core. Note that to run this example problem, MPACT uses eight cores, whereas Shift can run on however many extra processors are requested.

\section{Listing 11. VERA CASL progression problem 3a Shift eigen- value input.}

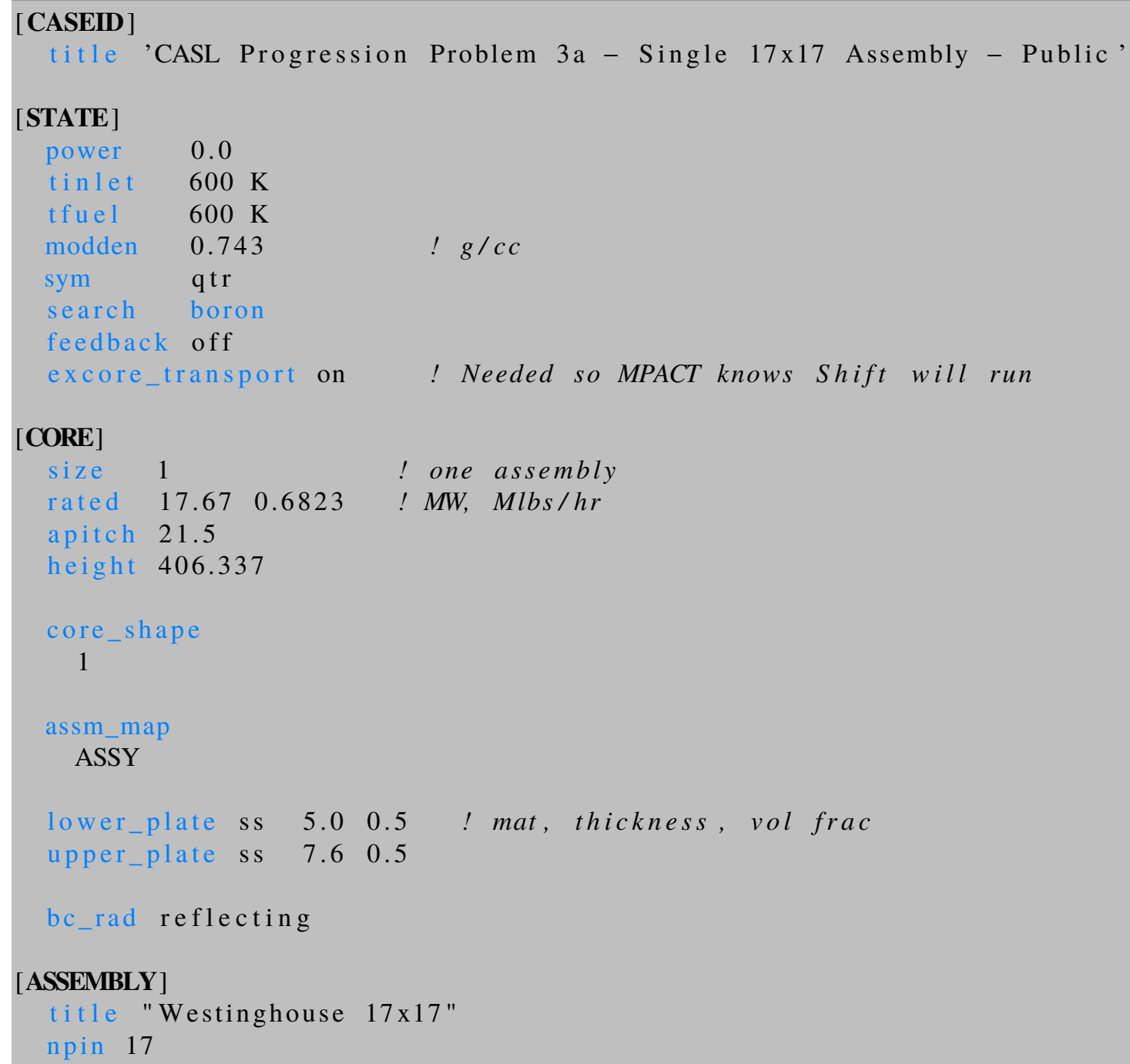




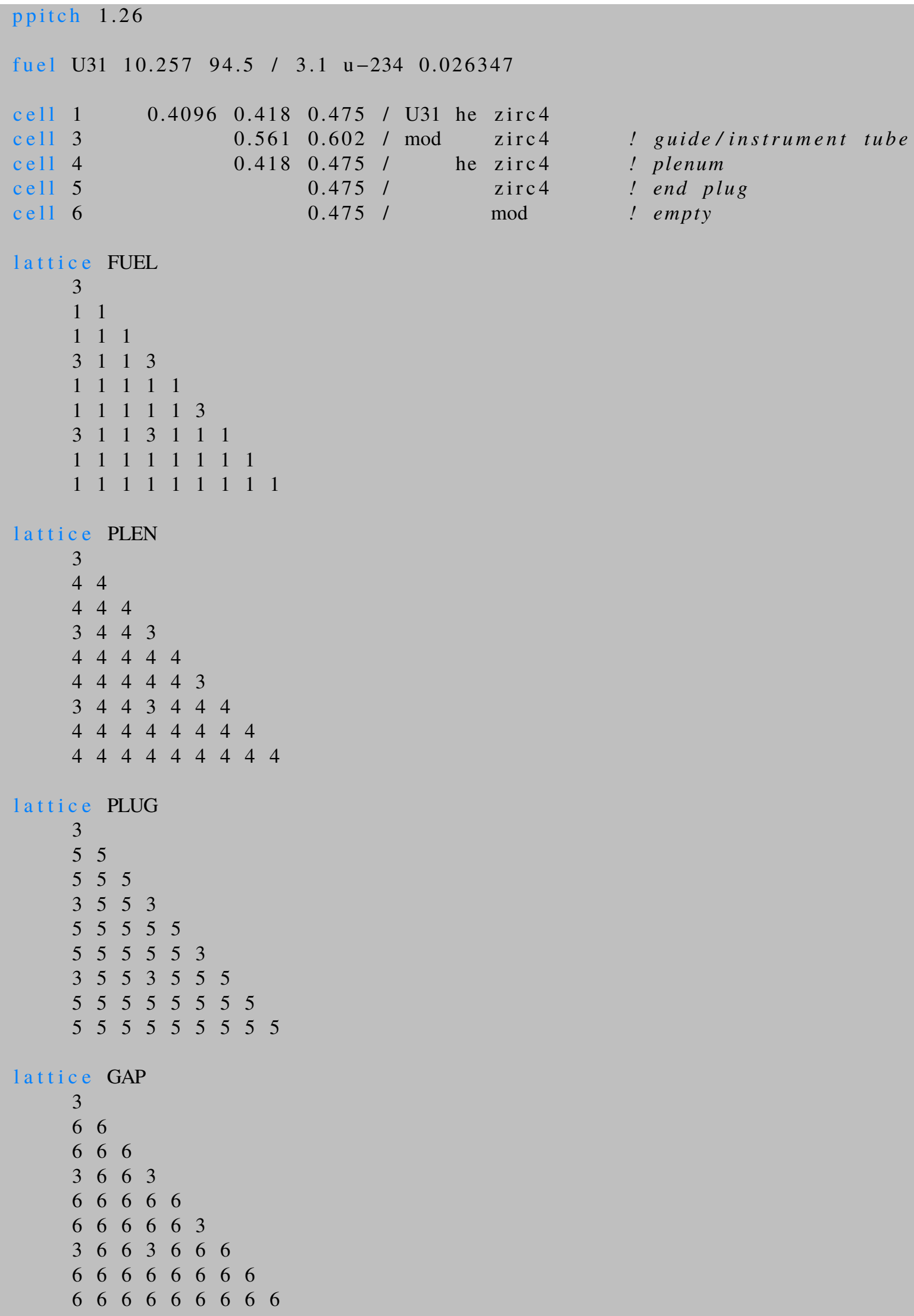




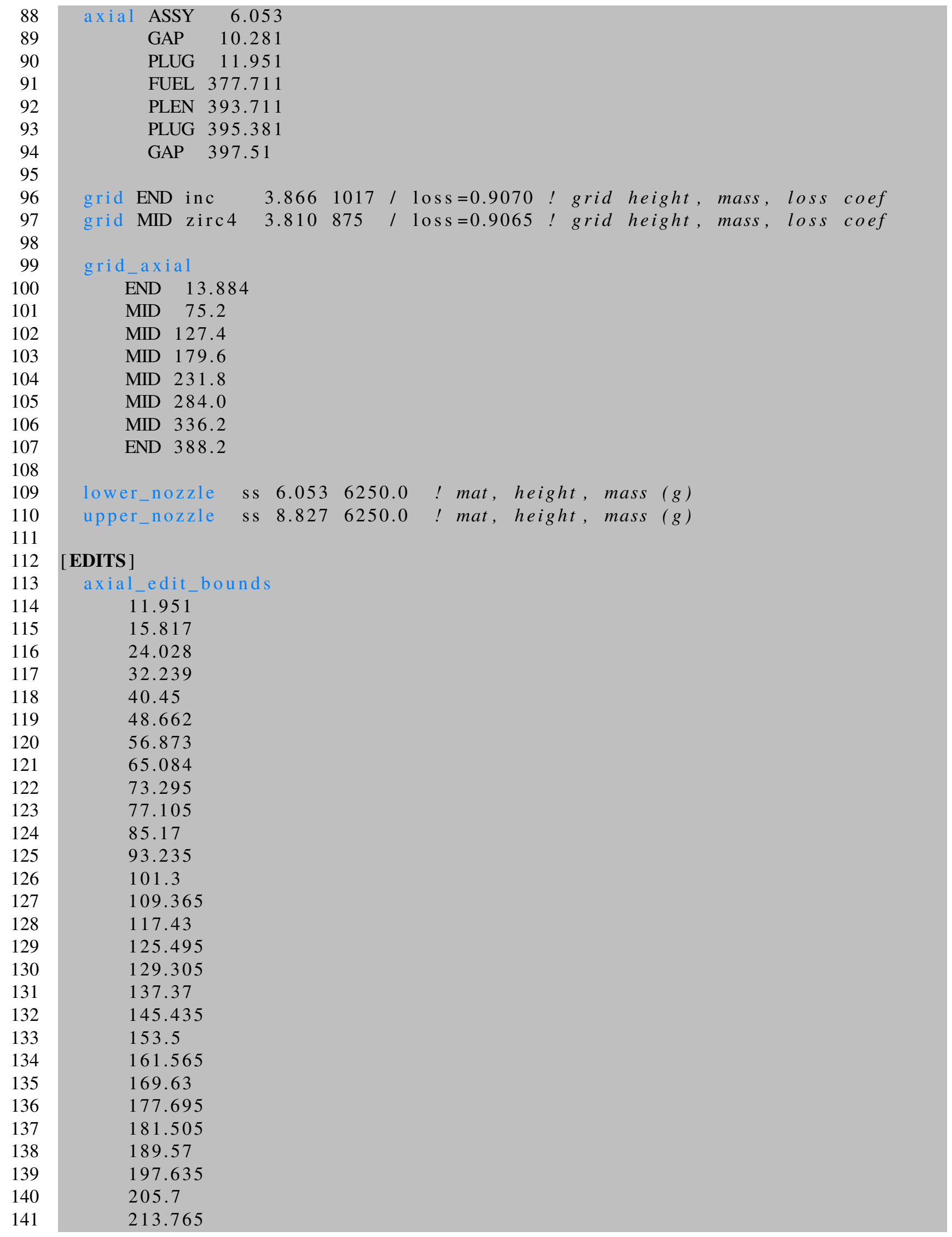


142

143

144

145

146

147

148

149

150

151

152

153

154

155

156

157

158

159

160

161

162

163

164

165

166

167

168

169

170

171

172

173

174

175

176

177

178

179

180

181

182

183

184

185

186

187

188

189

190

191

221.83

229.895

233.705

241.77

249.835

257.9

265.965

274.03

282.095

285.905

293.97

302.035

310.1

318.165

326.23

334.295

338.105

346.0262

353.9474

361.8686

369.7898

377.711

\section{[MPACT]}

num_space $\quad 8$

num_angle $\quad 1$

num_threads 1

ray_spacing $\quad 0.08$

shield_ray_spacing 0.08

azimuthals_octant 4

shield_azimuthals_octant 4 polars_octant 1

! This library is only for testing

xs_filename mpact8g_70s_v4.0m0_02232015.fmt

res_up_scatter false

\section{[SHIFT ]}

$\mathrm{Np} \quad 1000000$

num_cycles 1000

num_inactive_cycles 300

output_fission_source true

output_geometry true

global_log debug

problem_mode eigenvalue

! tally $d b$

n_bounds $\quad 2.0 \mathrm{e} 7 \quad 1.0 \mathrm{e} 6 \quad 1.0 \mathrm{e} 4 \quad 1.0 \mathrm{e} 2$

! nuclides to tally reactions for in ZAID format

micro_zaids 922389223594239

! ENDF MT values

micro_rxns $\quad 1 \quad 18 \quad 27$ 


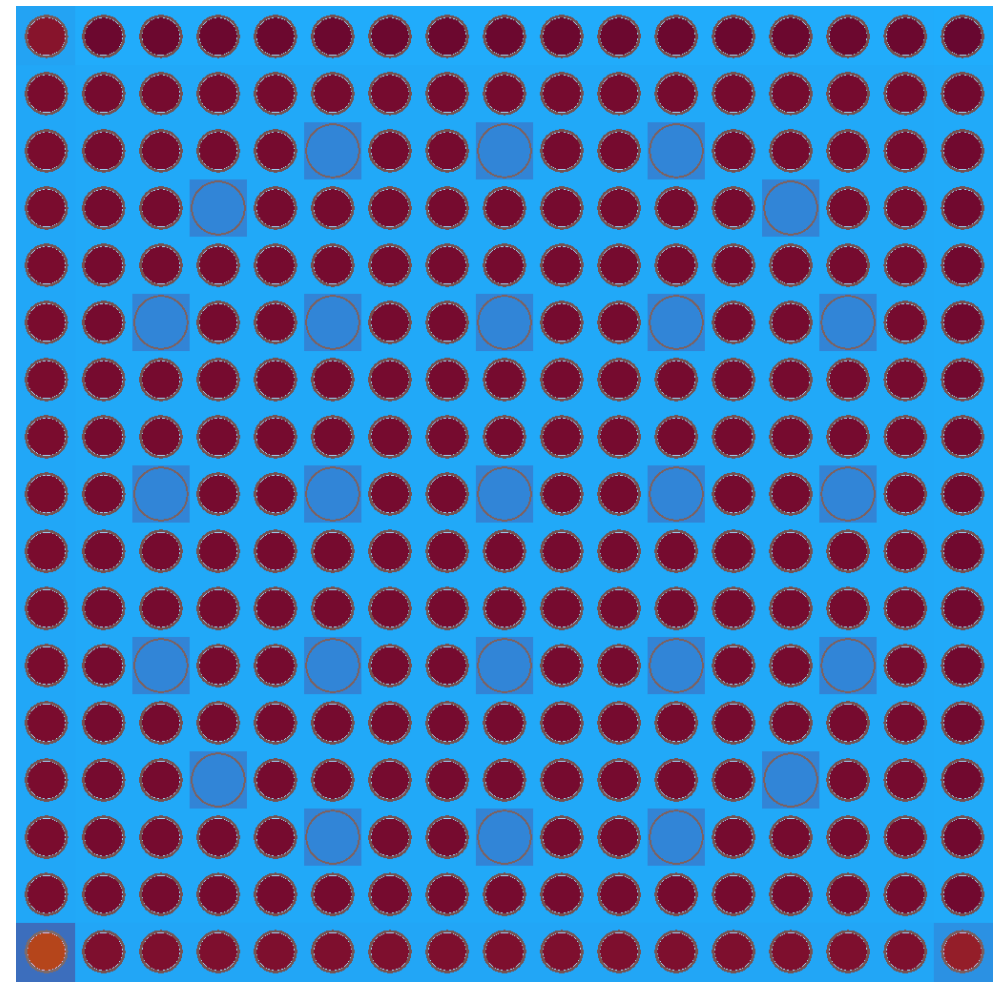

Figure 4. Ray trace of the progression problem 3a geometry at the axial midplane.

\subsection{SHIFT FORWARD MINICORE EXAMPLE}

This next example is a minicore version of CASL progression problem 9 [10], with Shift running in forward mode to calculate vessel fluence. The number of depletion states has been reduced for this example from the original problem.

Listing 12 shows the explicit VERA input with Shift set to run in forward mode. In the SHIFT block, a vessel fluence tally is set up in the barrel, pads, liner, and vessel. The neutron bounds specified are used in Shift to tally the energy-binned flux in these regions. Even though this problem has quarter core symmetry, num_theta refers to the number of bins over the full $360^{\circ}$. This number of angular bins must be divisible by four for quarter core symmetry problems. Note that although feedback is on, the temperatures and densities are not set to be transferred from MPACT to Shift because unique pins are turned off. This problem is set up to run MPACT on 32 cores, whereas Shift can use however many cores the user designates. A ray trace of the geometry midplane created by Shift is shown in Fig. 5.

\section{Listing 12. VERA CASL progression problem 9 minicore input.}

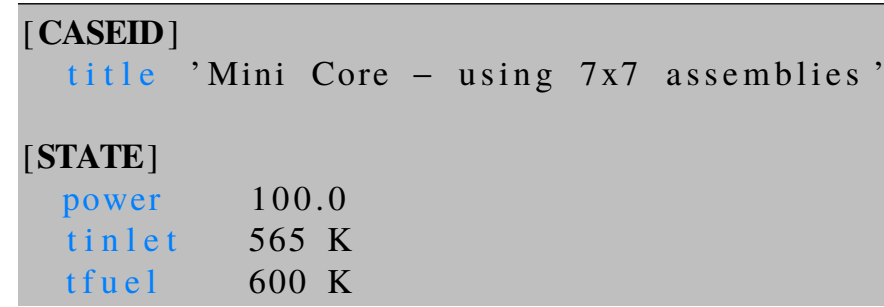




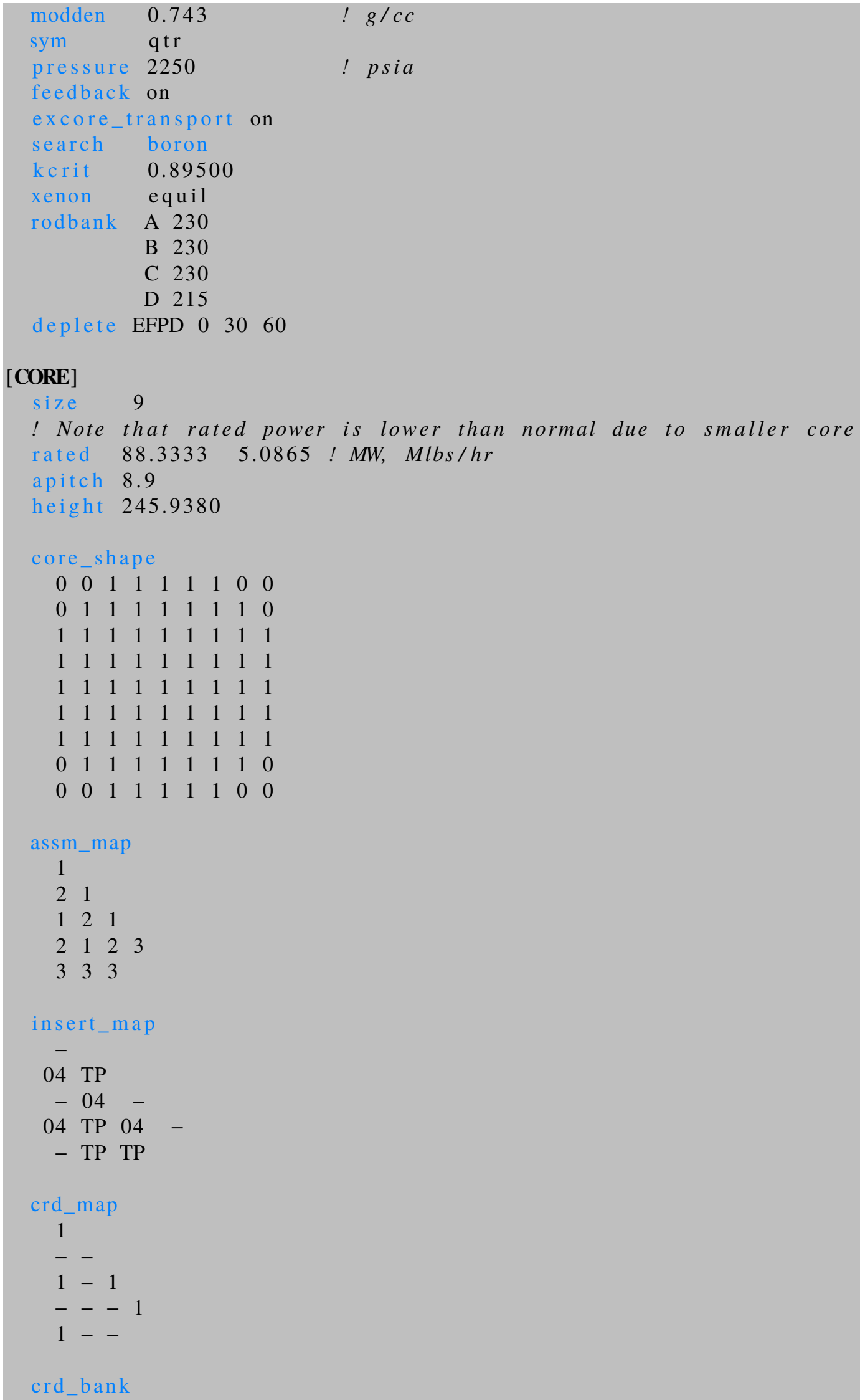




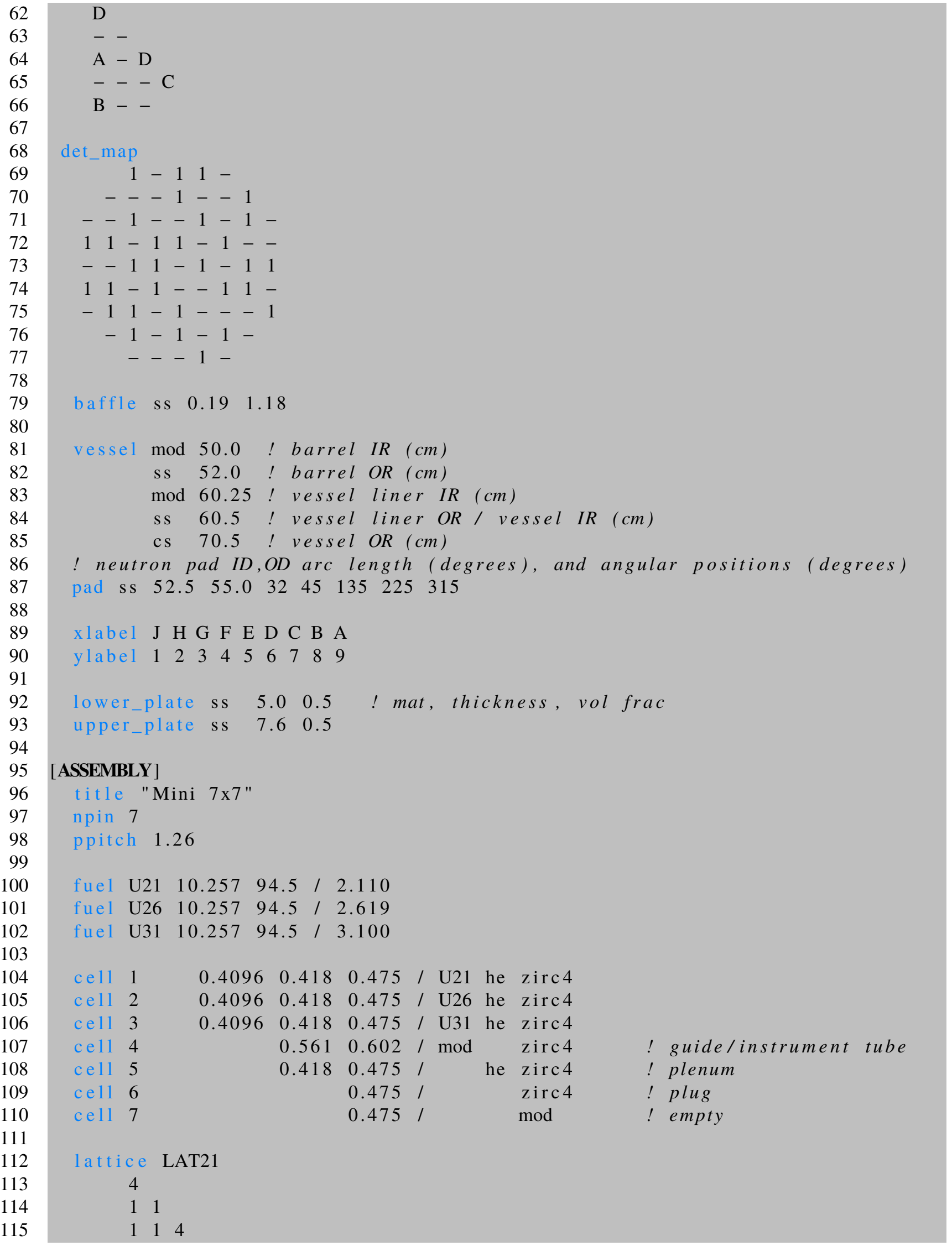


116

117

118

119

120

121

122

123

124

125

126

127

128

129

130

131

132

133

134

135

136

137

138

139

140

141

142

143

144

145

146

147

148

149

150

151

152

153

154

155

156

157

158

159

160

161

162

163

164

165

166

167

168

169

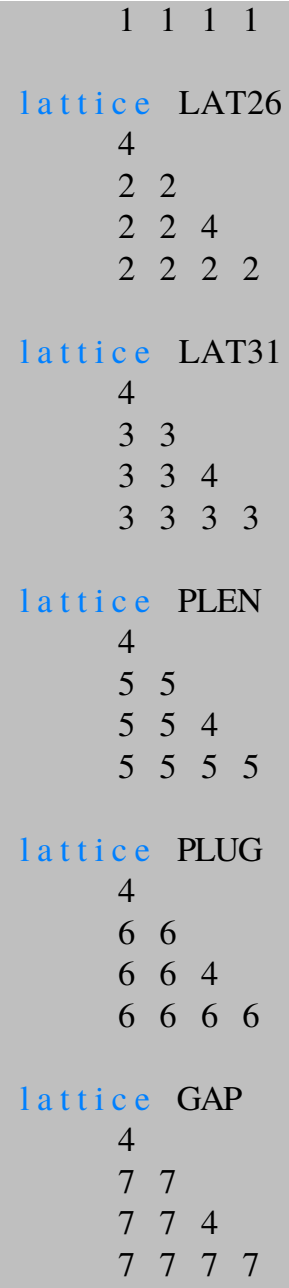




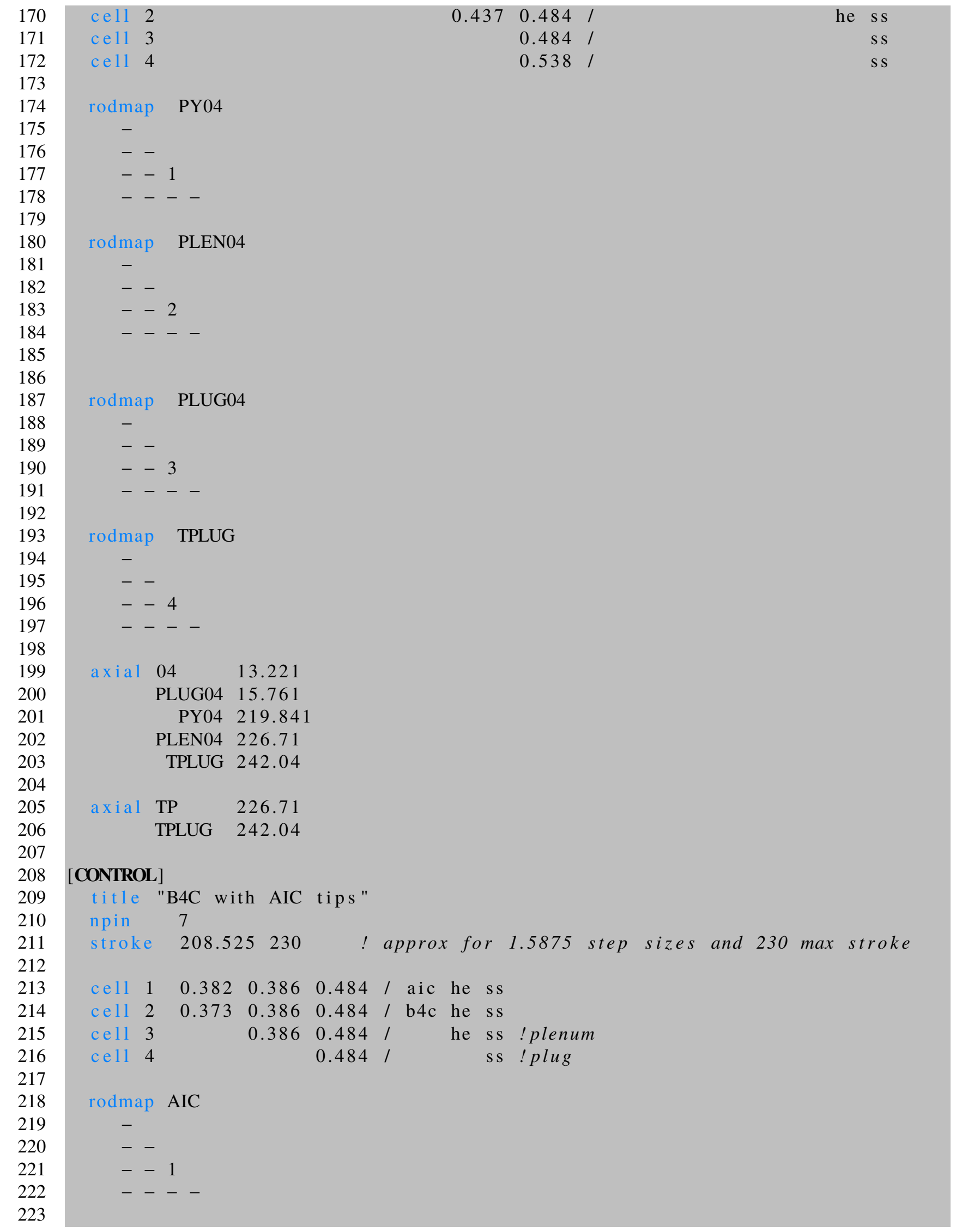


253

254

255

256

257

258

259

260

261

262

263

264

265

266

267

268

269

270

271

272

273

274

275

276

277

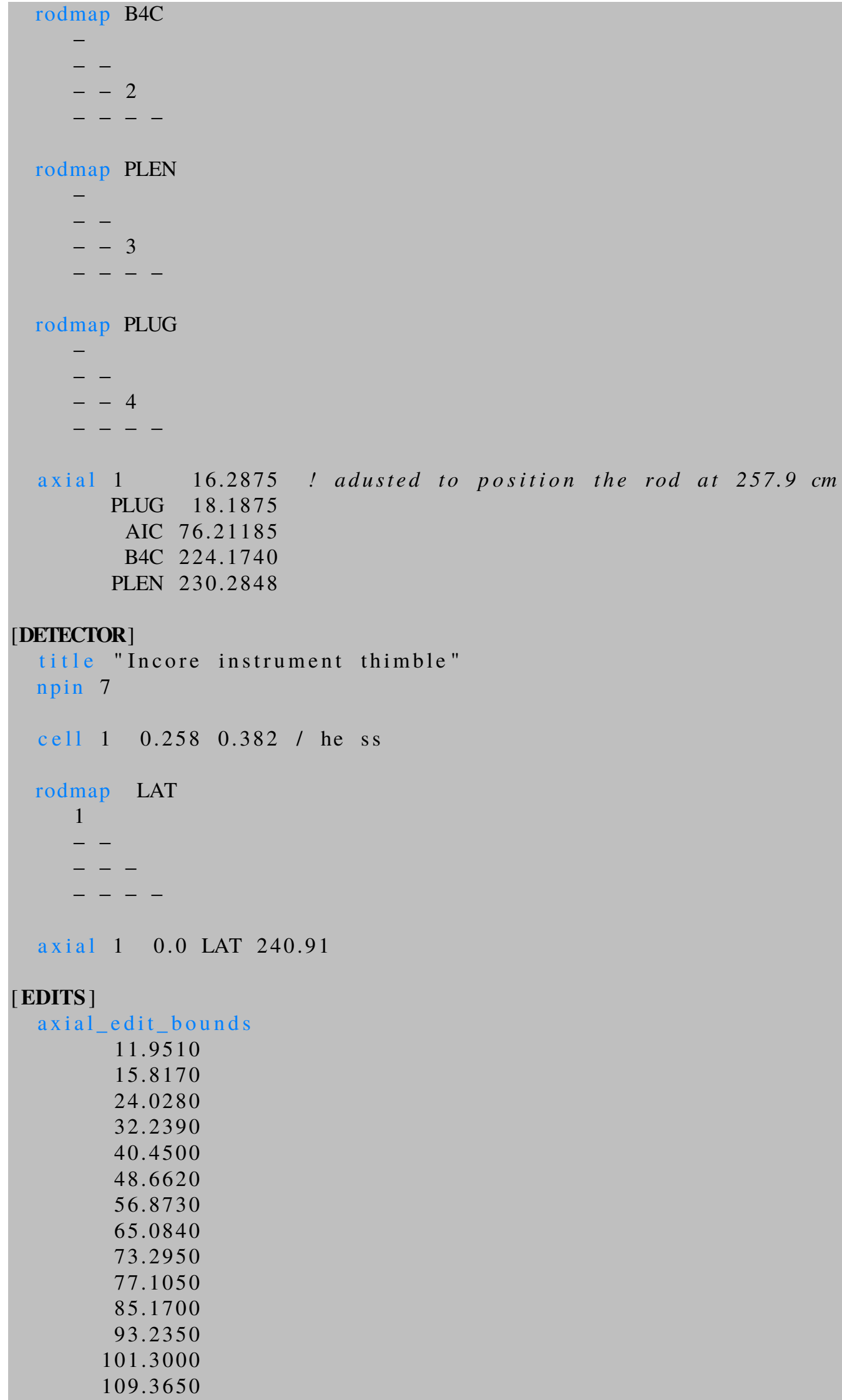



117.4300
125.4950
129.3050
137.3700
145.4350
153.5000
161.5650
169.6300
177.6950
181.5050
189.4262
197.3474
205.2686
213.1898
221.1110

\section{[MPACT]}

num_space $\quad 32$

num_angle $\quad 1$

num_threads $\quad 1$

ray_spacing $\quad 0.08$

shield_ray_spacing 0.08

azimuthals_octant 16

polars_octant 2

res_up_scatter false

dep_substep $\quad 1$

k_tolerance $\quad 1 \mathrm{e}-5$

flux_tolerance $\quad 5 e-5$

\section{[SHIFT]}

problem_mode forward

$\mathrm{Np} \quad 1$ e 10

output_geometry true

output_fission_source true

create_unique_pins false

transfer fiss_src

! tally_db

n_bounds $\quad 2.0 \mathrm{e} 7 \quad 1.0 \mathrm{e} 6$

num_theta 64

num_axial 10

radial_mesh $0 \begin{array}{llllllllll} & 49.99999 & 50.25 & 50.5 & 50.75 & 51 & 51.25 & 51.5 & 51.75 & 52.00001\end{array}$

$\begin{array}{lllllllll}60.249999 & 60.50001 & 61.5 & 62.5 & 63.5 & 64.5 & 65.5 & 66.5 & 67.5\end{array}$

$\begin{array}{lll}68.5 & 69.5 & 70.50001\end{array}$

\subsection{CADIS VESSEL FLUENCE MINICORE EXAMPLE}

This example is the same problem as the previous example, but Shift is set up to run in CADIS mode. Listing 13 only shows the SHIFT block since those are the parameters that change relative to the previous example. Several parameters should be noted. First, the decomposition specified by the number of blocks in $i$ and $j$ gives a total number of processors for the Shift calculation of 25. The xs_library parameter specifies the multigroup library used for the adjoint deterministic transport calculation to produce variance reduction parameters. The num_groups and new_grp_bounds 


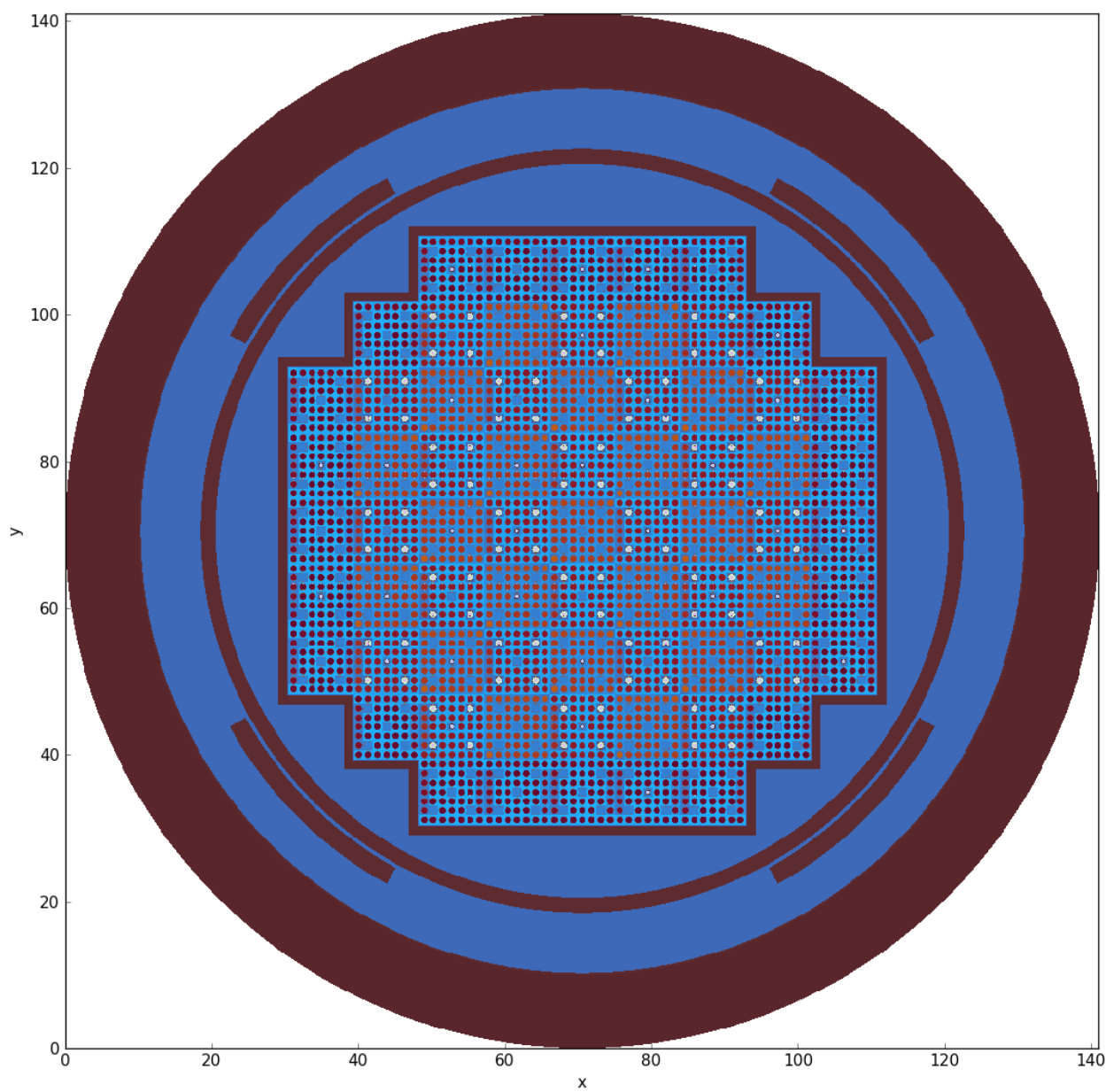

Figure 5. Ray trace of progression problem 9 geometry at axial midplane.

parameters indicate the energy resolution that the adjoint deterministic calculation uses. In this example, the multigroup library has 56 groups but is collapsed to 8 groups for efficiency, less memory use, and to target higher energy regimes for ex-core regions. The deterministic calculation is run with cell homogenized cross sections in each pin cell with flux-weighting when the cell_homogenize parameter is true. Otherwise, the cross sections are volume weighted in each computational cell, which is less time consuming. The verbosity parameter controls how much information from the deterministic adjoint solver is printed to the screen at each iteration.

Listing 13. VERA CASL progression problem 9 minicore

\section{CADIS input.}

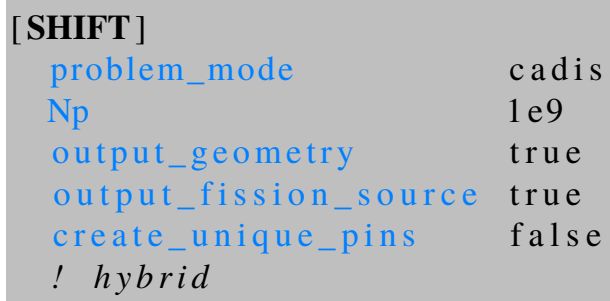




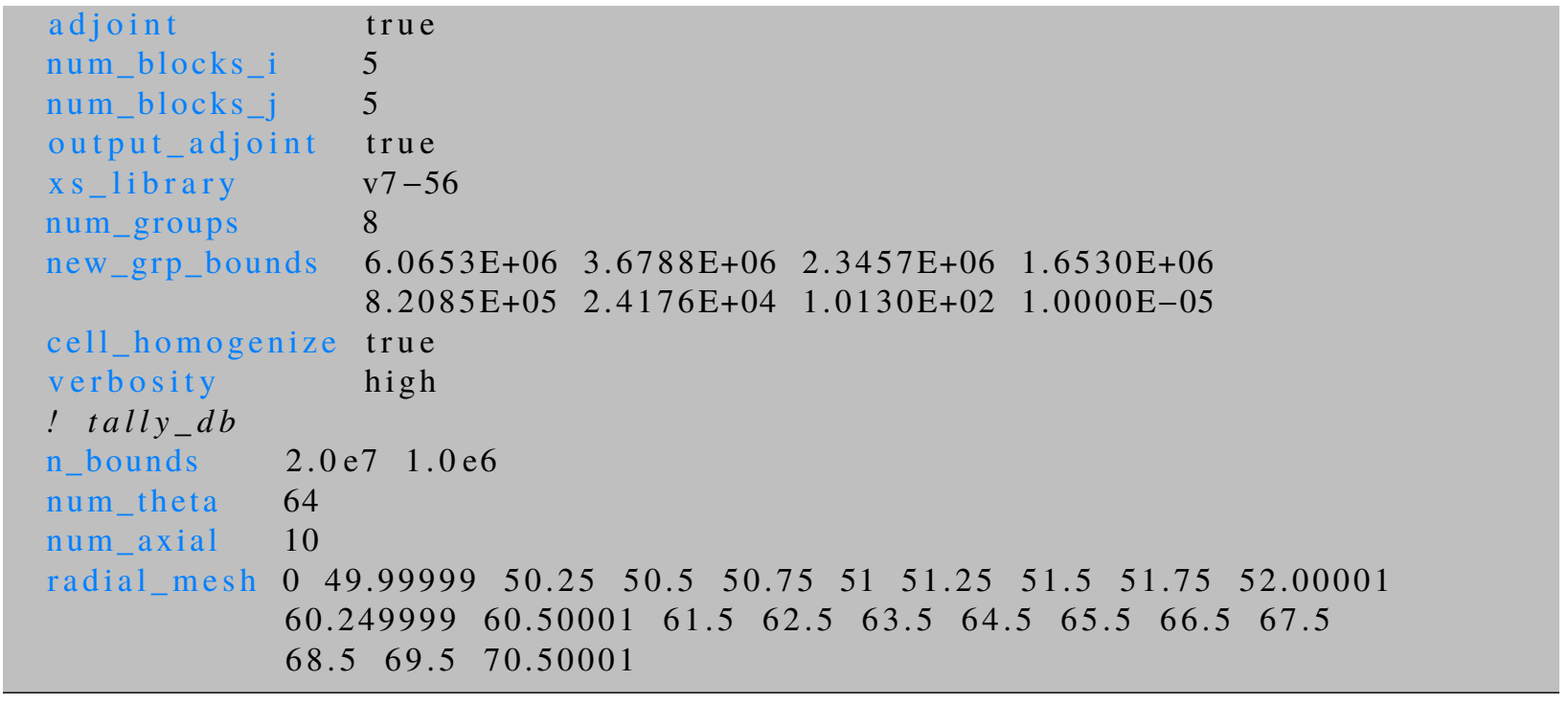

\subsection{AUTOMATED DETECTORS EXAMPLE}

This example is based on the previous two examples and demonstrates use of the VERA input to automatically generate a supplemental ex-core file with detectors and bioshield. Listing 14 shows the relevant sections of the CORE and SHIFT blocks for this example. A ray trace through the core midplane of this geometry is shown in Fig. 6. The supplemental ex-core input file is created when the user runs the react $2 \mathrm{xml}$ script with a VERA input file. This supplemental file takes the base of the VERA input filename with an extension of . omn. The user must then run excore $2 x m l$ with the *. omn file to convert it to $\mathrm{xml}$ format (as is the case with any calculation using a supplemental ex-core file).

The user should note that this example does not specify the num_blocks_i and num_blocks_j parameters, so the default values of ten for each are used; therefore Shift would run on 100 processors. Also, if running this example through verarun, as described in $\S 3.1$, the excore $2 x m l$ script is run automatically and an error is thrown if the excore_filename parameter does not specify the correct filename.

\section{Listing 14. VERA CASL progression problem 9 minicore input} with automated excore detectors.

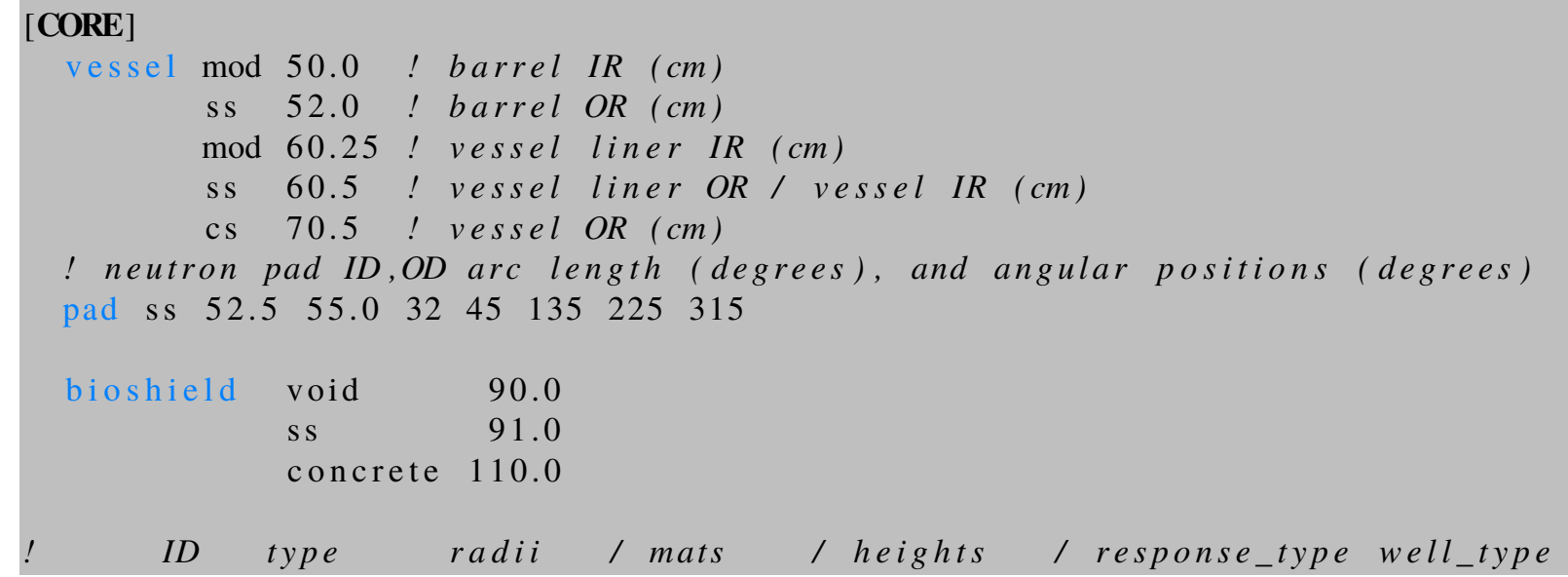


Ex-core Modeling with VERA User Manual

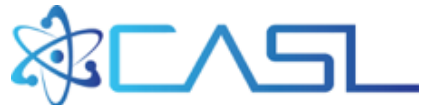

15

16
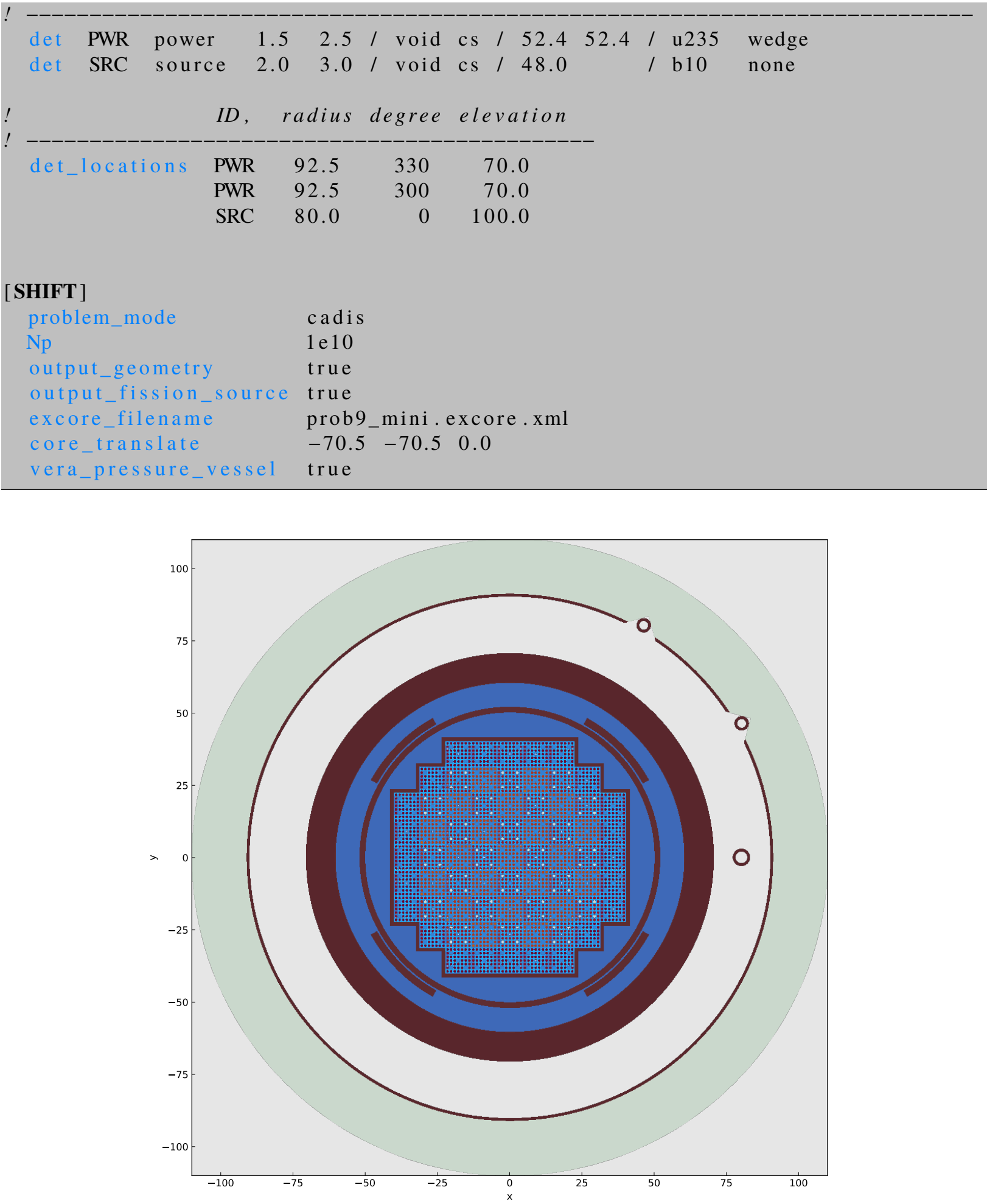

Figure 6. Ray trace of example geometry using the automated generation of the supplemental ex-core file with bioshield and detectors.

Several parameters shown in Listing 14 should be noted. 
- The bioshield and detector materials specified after the bioshield and det keywords must be defined in the template file located in the VERA source code (scripts/Init/omnibus_template . txt) and given in Appendix A.

- For the det parameter, the response_type options are b10 or u235, and the well_type options are none or wedge (as shown in Table 5).

- This example defines two different detector types with IDs of $P W R$ and $S R C$, where the $P W R$ detector uses the wedge-shaped well.

- The placement of the user-defined detector types in the ex-core region is given by the det_locations parameter. Detectors must be located outside of the vessel. The angle specified is specified as clockwise from the positive $x$-axis. For quarter core problems, this needs to be noted since Shift plots and models the geometry as the northeast quadrant.

- The user needs to ensure that the excore_filename parameter in the SHIFT block matches the actual name of the supplemental ex-core file after it is converted to XML format.

- In most cases, the user should have vera_pressure_vessel set to true. If this parameter is not set, the VERA geometry extends to the outer radius of the barrel and the supplemental ex-core file defines the geometry beyond it. When using the bioshield parameter, most users should use the full vessel geometry defined in the VERA input file by the vessel parameter.

In addition to the ex-core geometry, the generated supplemental ex-core file also includes a tally for the innermost volume of each specified detector. For this example, each $P W R$ detector has two tallies, one for each axial extent. When running in CADIS mode, this can require the user to inspect the *.omn file to determine the name of the tally to optimize and add it to the hybrid_tally_names parameter. To reduce the burden on the user, there are several ways to automatically populate hybrid_tally_names in the XML version of the VERA input:

1. If no hybrid_tally_names parameter is given in the SHIFT block, the parameter will automatically be populated with all ex-core detector tallies.

2. If the user gives the name of a detector ID, such as $P W R$ or $S R C$ in Listing 14, for the hybrid_tally_names parameter, the generated VERA XML input will replace the detector ID with the appropriate tally names for all instances of that detector ID.

3. If hybrid_tally_names contains a tally name(s) that does not match a detector ID, the parameter will be left unchanged in the VERA XML file and the user must ensure that it matches the name of a tally defined in the supplemental ex-core file.

This capability is under active development, and additional features will be available in future releases of VERA.

\subsection{EX-CORE FILE DETECTOR EXAMPLE}

Building on $\S 5.4$, this example demonstrates the ability to provide a manually defined supplemental ex-core input file defining the external geometry and tallies for the Shift calculation. The example 
input shown here is a modified mini version of CASL progression problem 5a [10]. All of the geometry outside of the core barrel is manually defined in the supplemental ex-core input file. Figure 7 shows a ray trace of this example geometry at the axial midplane.

Listing 15 shows the explicit VERA input with Shift set to run in CADIS mode. In the SHIFT block, the name of the ex-core file is specified. Also, since the ex-core geometry is defined with the origin at the center of the core, the VERA core must be translated by the radius of the outer barrel radius (additional details in Appendix C). Note that if the parameter vera_pressure_vessel was set to true, the translation would correspond to the outermost vessel radius. The final item to note from this input block is the definition of the boundary mesh. As noted in the comments in the example, this boundary mesh serves to cut the full geometry, including the ex-core part, to the northeast corner with reflecting boundaries on the negative $\mathrm{x}$ and negative $\mathrm{y}$ boundaries (to simulate a quarter core). Also, the coordinates of the boundary mesh box are based on the ex-core geometry origin and not the VERA origin.

Listing 16 shows the explicit supplemental ex-core input defining the ex-core geometry, tallies, and compositions for the Shift calculation. For most of the entries in this ex-core file, details can be found in the Shift manual [12]. One entry is specific for the geometry section of the ex-core file: the core universe. This indicates the core geometry is taken from VERA and is inserted with the fill parameter in the hole universe that is defined in the ex-core file. The composition materials must also be defined to correspond to the materials in the ex-core geometry; this example shows dummy materials. Currently, the user cannot use any of the materials that were defined in the VERA input for ex-core geometry cells. Finally, the tally block shows a cell tally set up in the detector cell for the flux and fission reaction rate. Since there were no neutron or photon bins specified, this tally will give the user the total (summation of neutron plus photon) flux and fission from neutrons only. This total flux is not useful for analysis so the user should definitely include neutron and photon bins for a flux tally if it applies to their problem of interest.

\section{Listing 15. VERA CASL progression problem 5 minicore VERA input using ex-core file.}

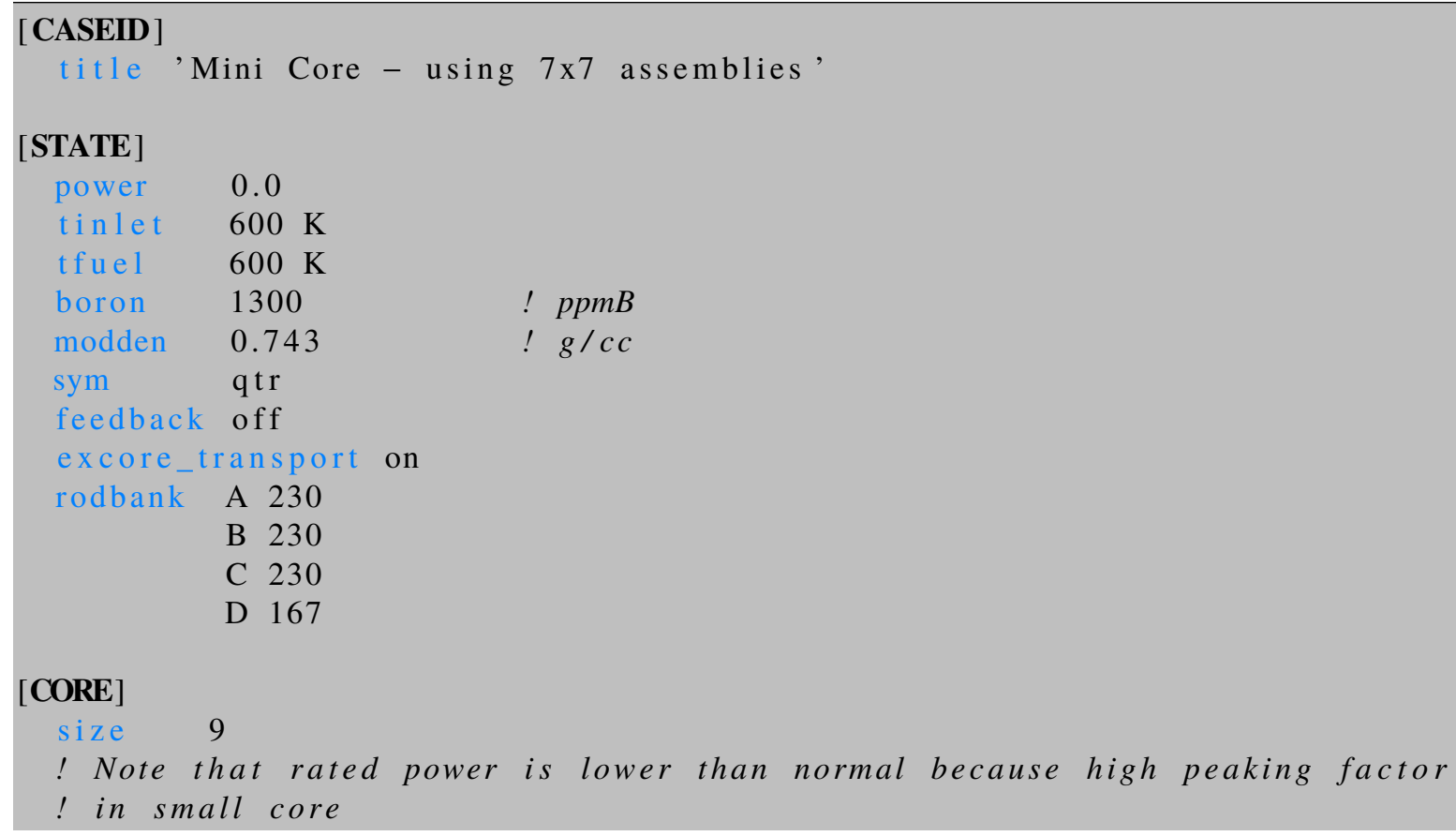




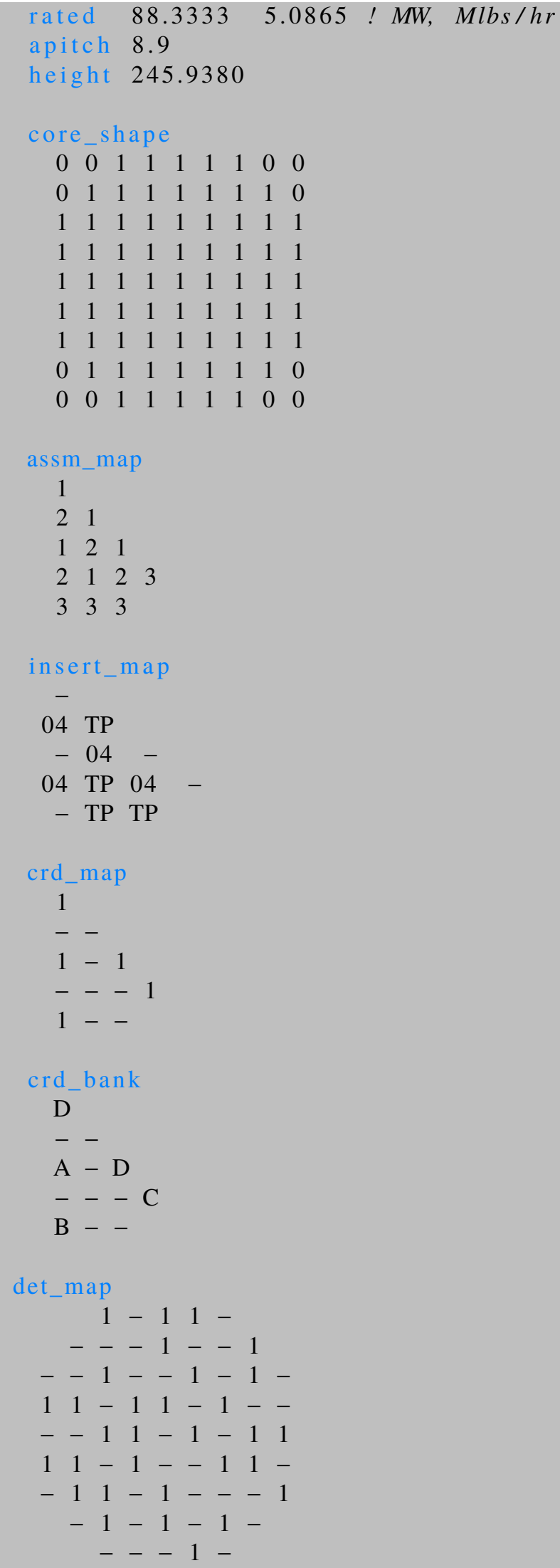




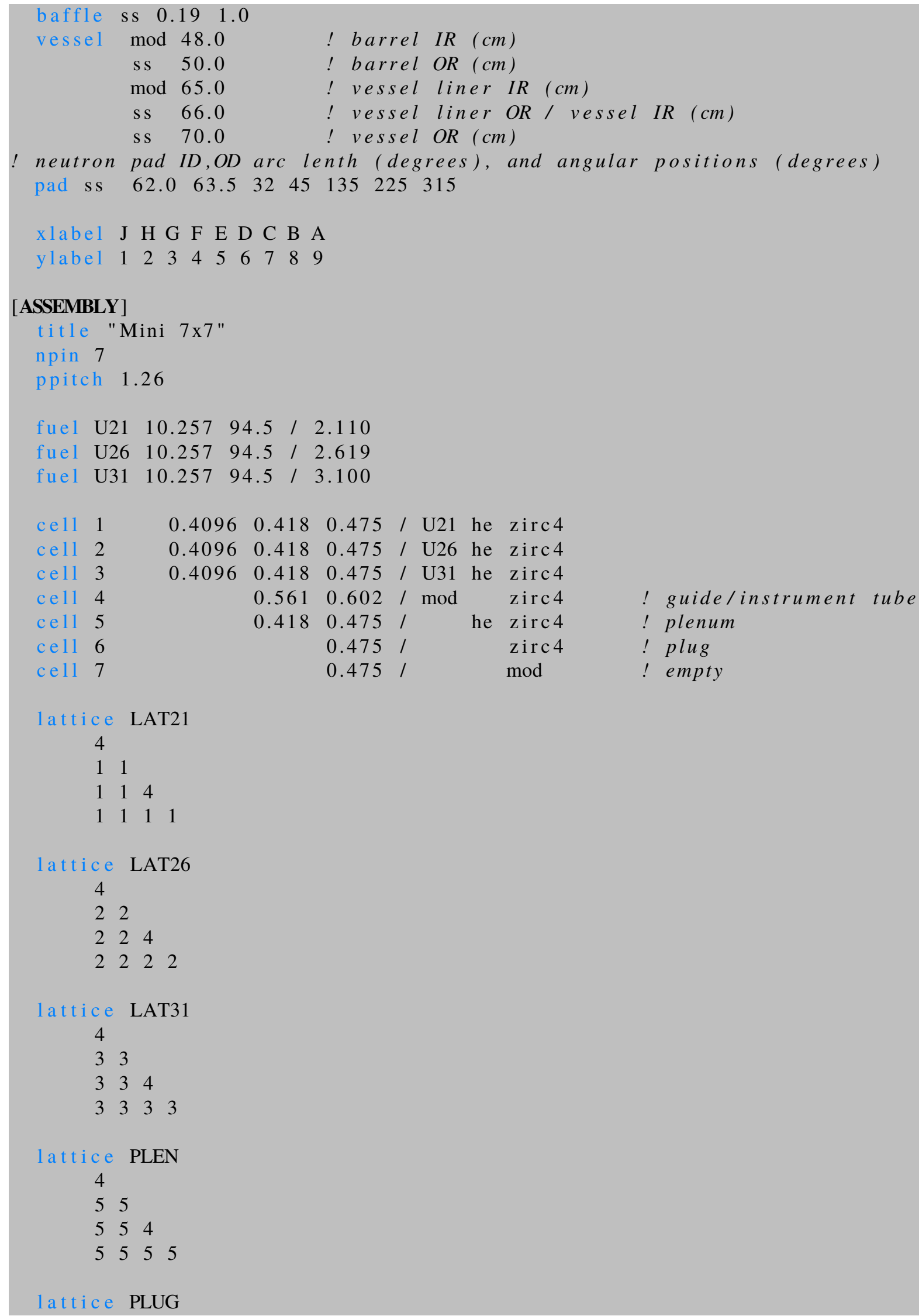




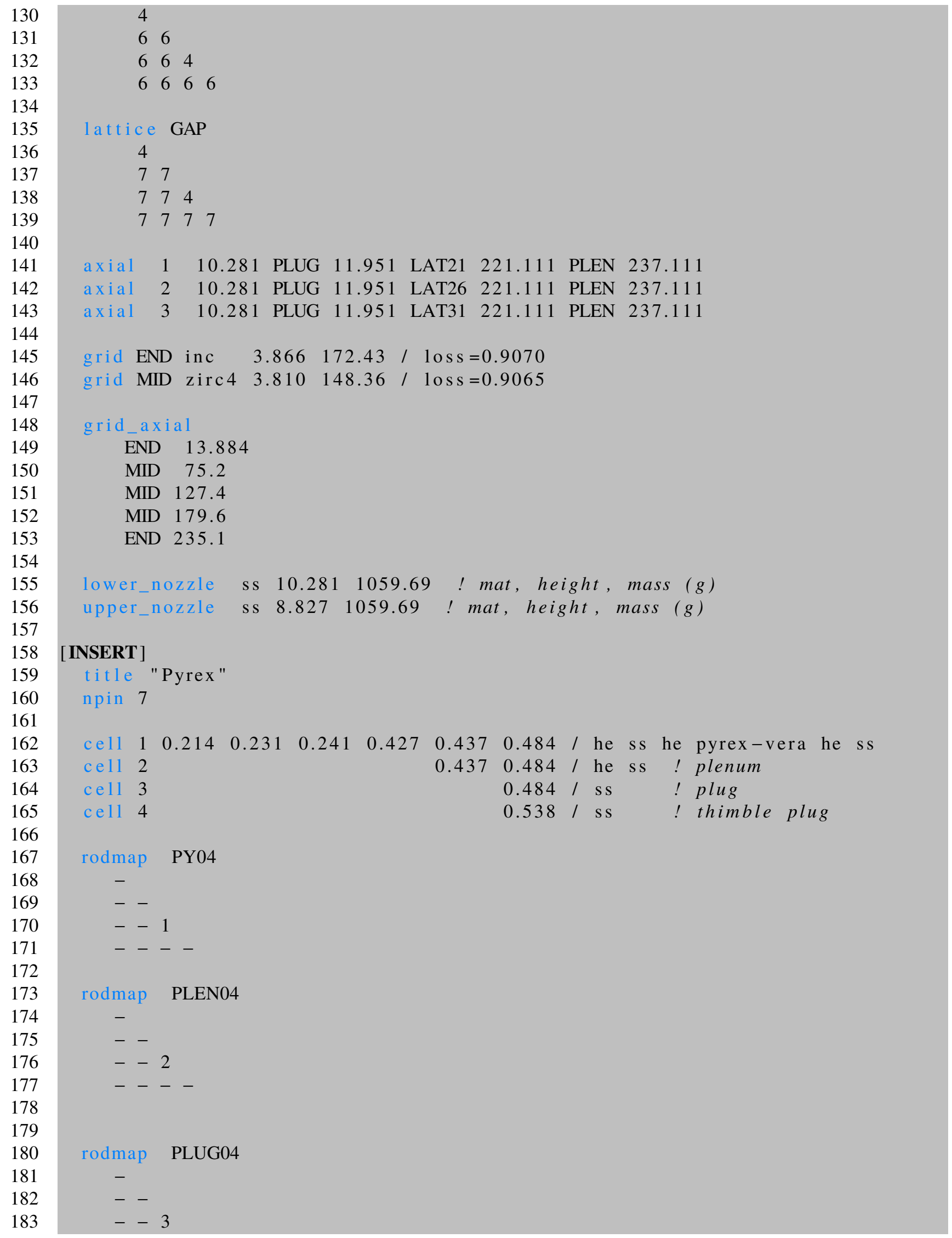


184

185

186

187

188

189

190

191

192

193

194

195

196

197

198

199

200

201

202

203

204

205

206

207

208

209

210

211

212

213

214

215

216

217

218

219

220

221

222

223

224

225

226

227

228

229

230

231

232

233

234

235

236

237

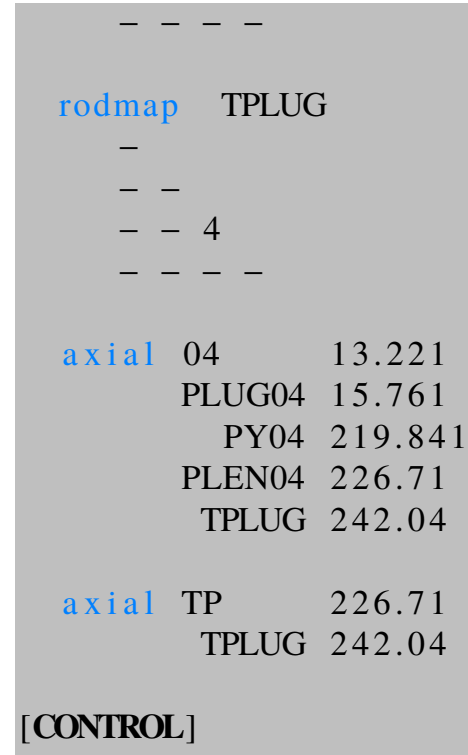




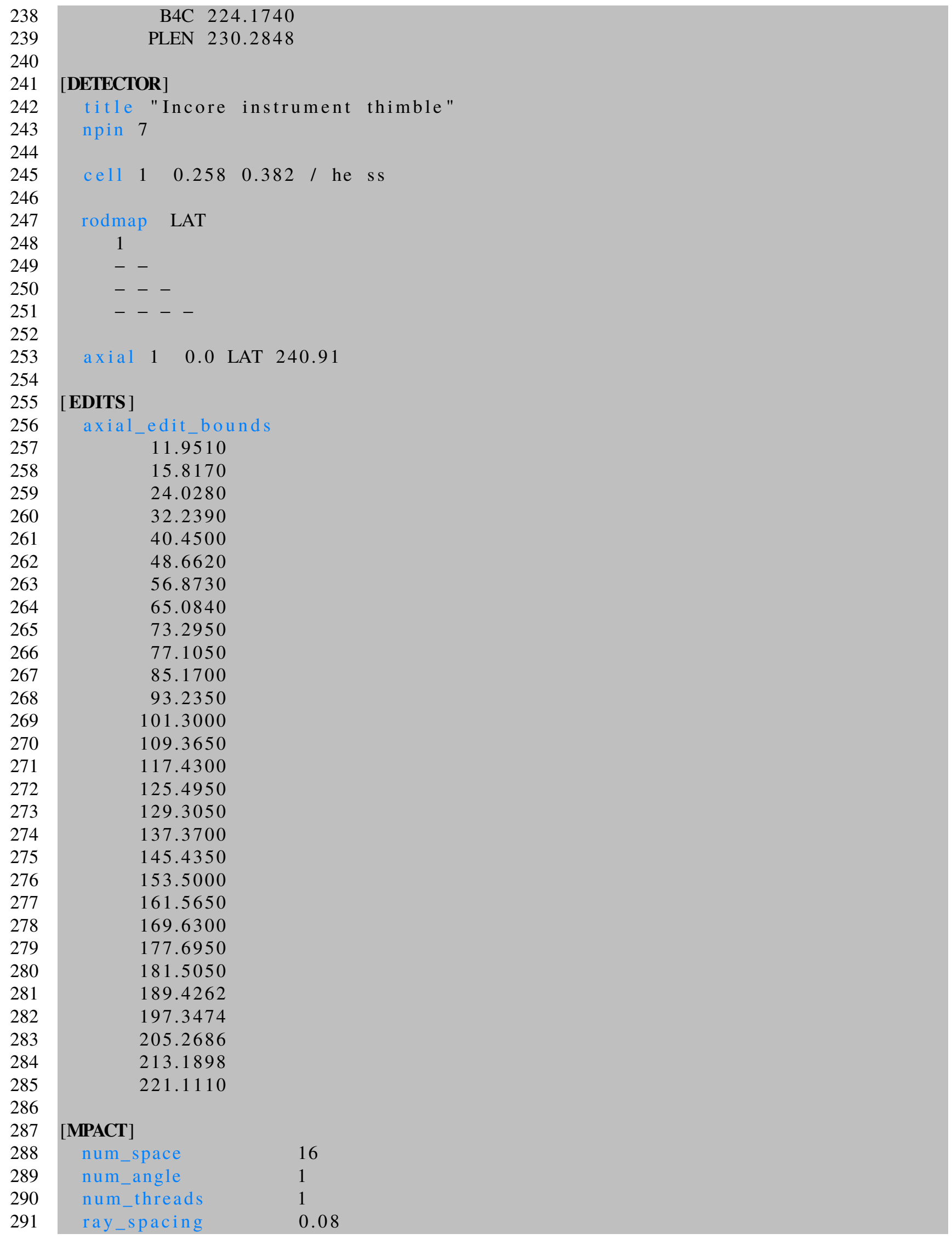




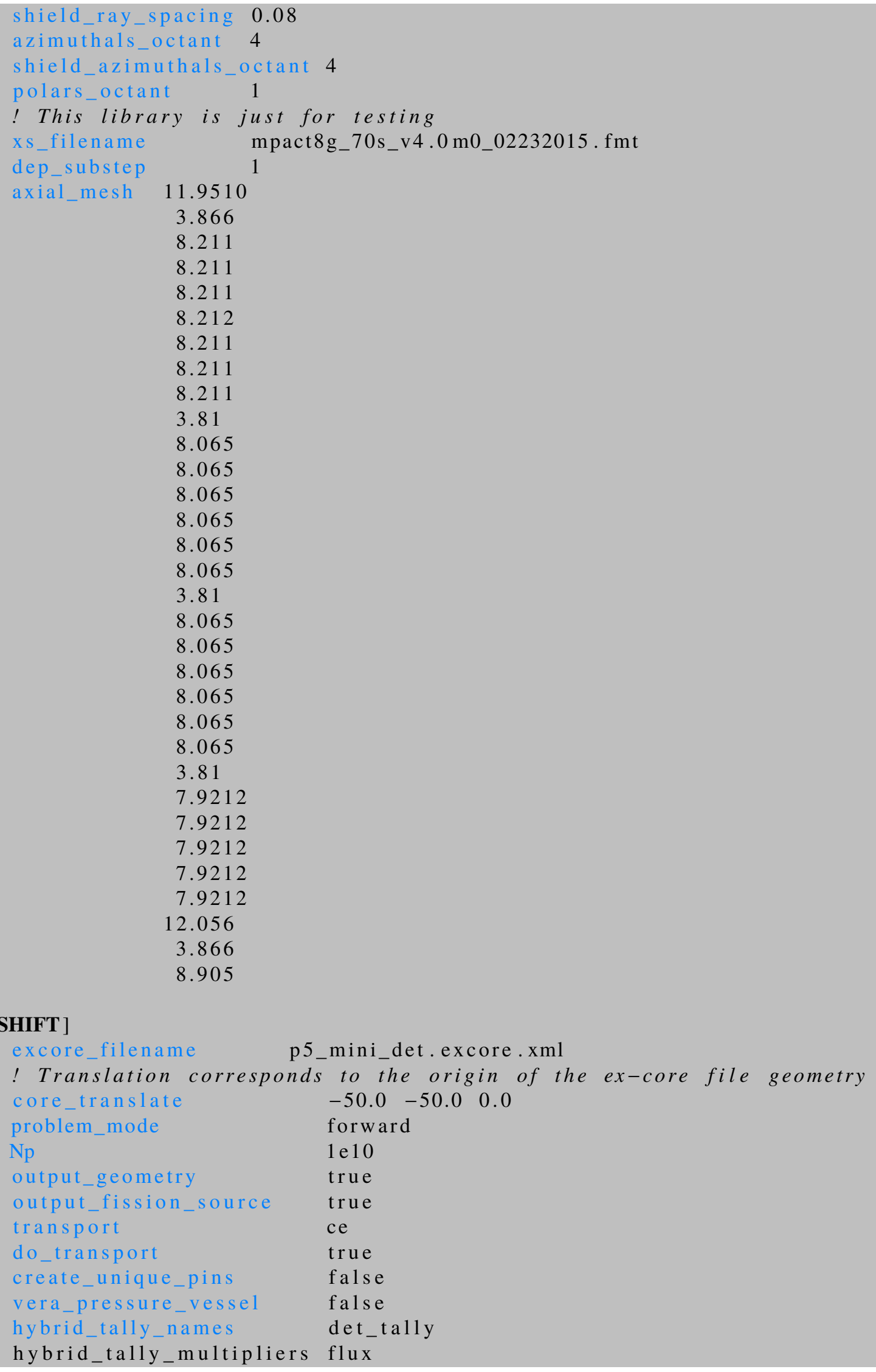

\section{[SHIFT]}


! This boundary mesh cuts the full ex-core geometry to the NE quarter bc_bnd_mesh reflect vacuum reflect vacuum vacuum vacuum

! The origin of this boundary mesh is based on the ex-core file geometry

x_bnd_mesh $0.0 \quad 85.0$

y_bnd_mesh $0.0 \quad 85.0$

z_bnd_mesh $0.0 \quad 260.0$ 
Listing 16. VERA CASL progression problem 5 minicore excore file input.

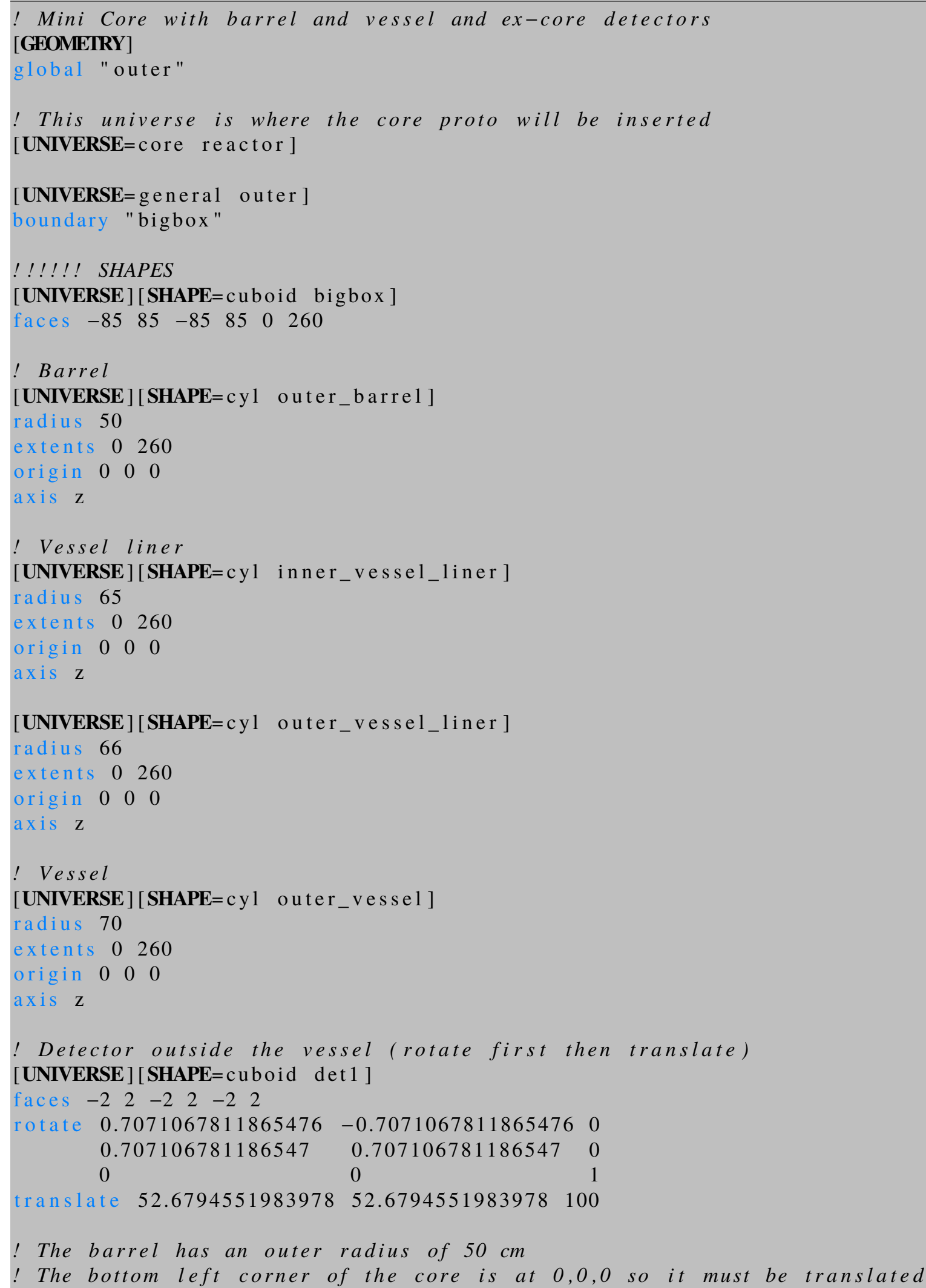


81

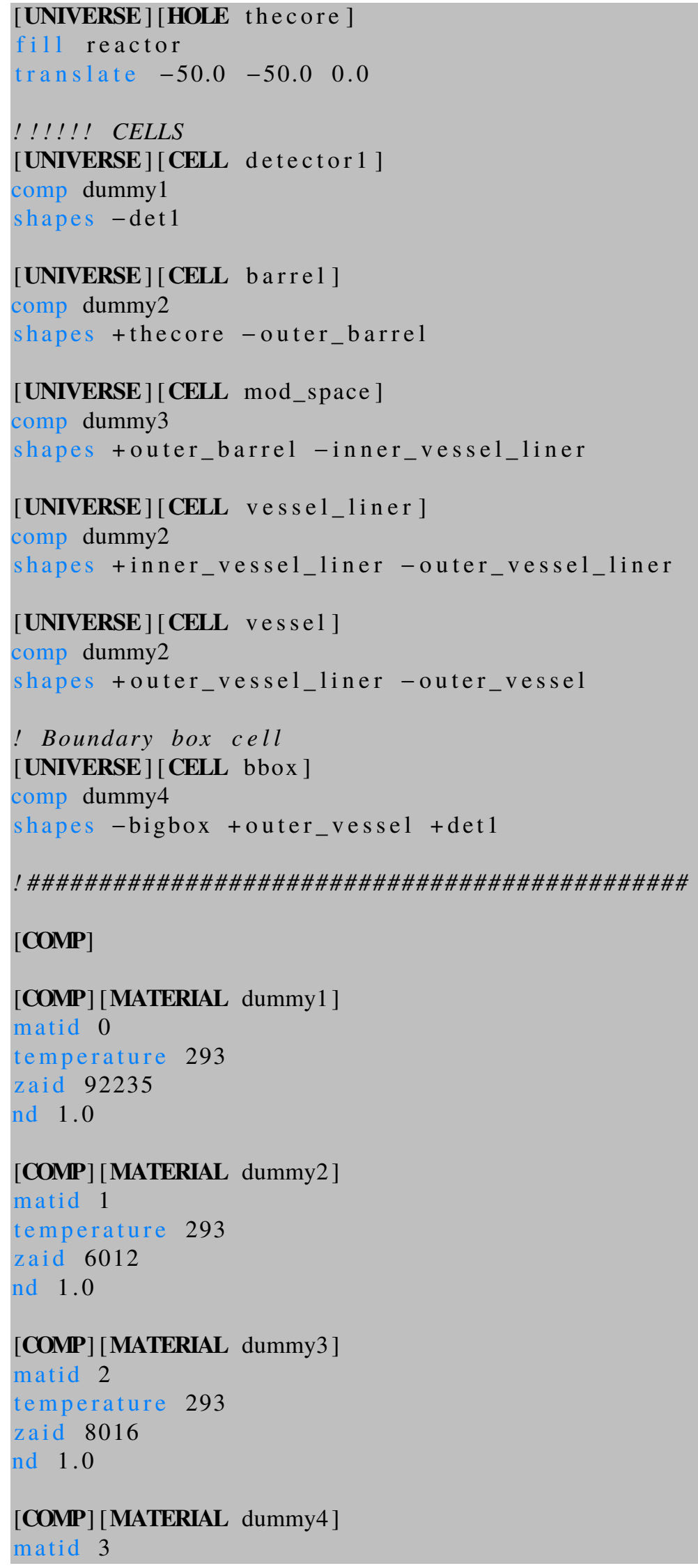



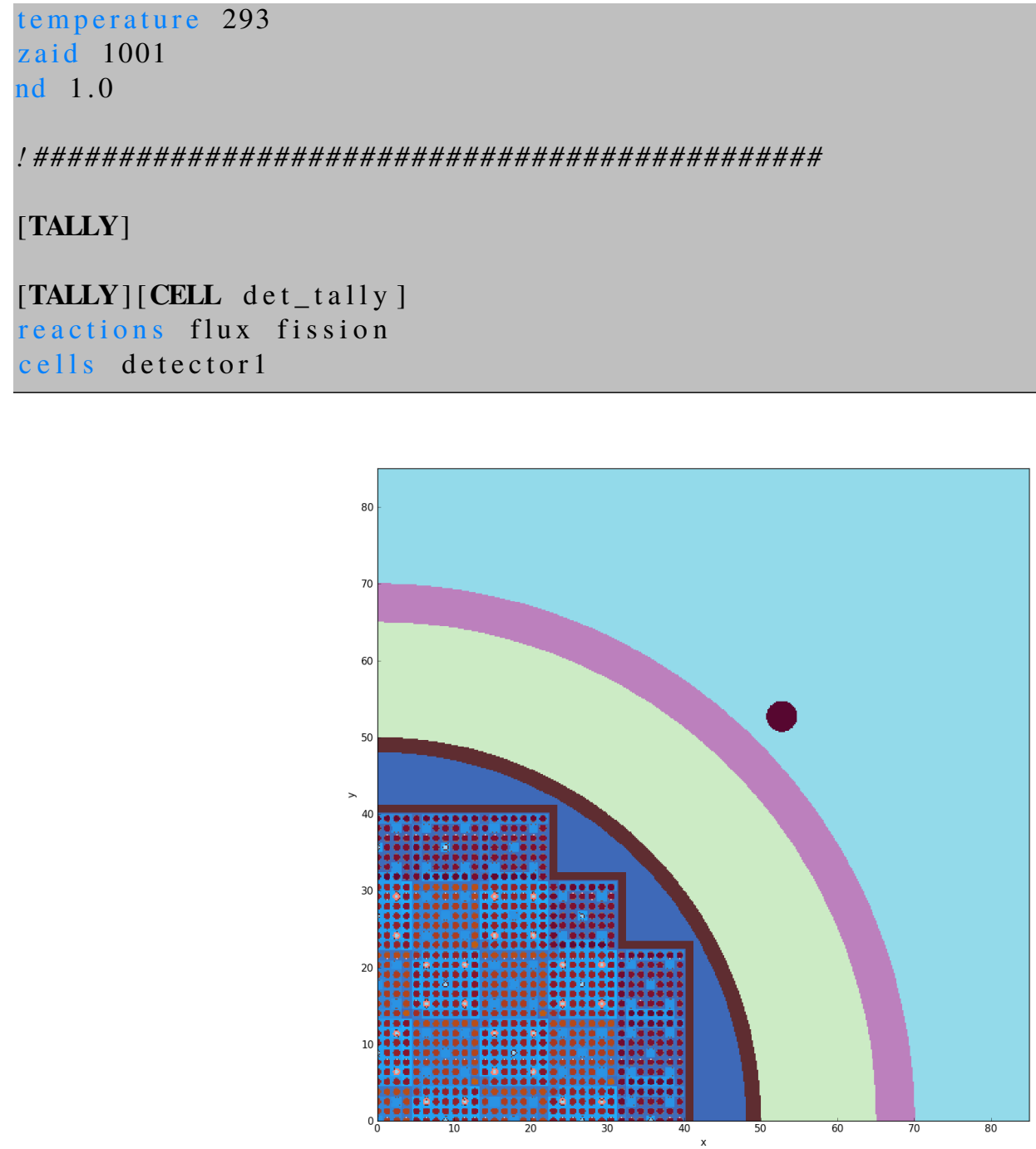

Figure 7. Ray trace of example at axial midplane with the excore regions defined manually with the supplemental ex-core input file. 


\section{ADVANCED AND EXPERIMENTAL FEATURES}

This section details the advanced postprocessing capabilities using Python with output from an ex-core calculation and the experimental core temperature homogenization options in Shift.

\subsection{CORE TEMPERATURE HOMOGENIZATION}

An experimental capability in VERA using Shift is the capability to investigate the effects of homogenizing temperature and coolant density regions of the core on ex-core quantities. These homogenization methods apply an average temperature in the fuel, clad, and coolant regions of a pincell depending on the method used. The input parameters governing these methods are given in Table 6. Four core temperature and density homogenization methods exist in VERAShift:

1. Assembly averaged: This method applies a volume-averaged temperature and density over each assembly in the core (shown in Fig. 8).

2. Assembly ring averaged: This method applies a volume-averaged temperature and coolant density in full assemblies encompassed by user-specified radial rings around the center of the core (shown in Fig. 9a).

3. Pin ring averaged: This method applies a volume-averaged temperature and coolant density to pins encompassed by user-specified radial rings around the center of the core (shown in Fig. 9b).

4. Explicit peripheral pins: This method must be used in conjunction with methods (1) or (2). It uses explicit temperature and coolant density in each pincell outside of a user-specified radial ring from the center of the core (shown in Fig. 10).

Full details about this capability and the investigation of its effect on vessel fluence can be found in Stocking [2, 13].

To use these experimental methods for ex-core calculations with VERA, please contact Tara Pandya (pandyatm@ornl.gov) at ORNL.

\subsection{ADVANCED POSTPROCESSING}

Besides the capabilities of VERAView, there are a lot of advanced Python postprocessing capabilities in Exnihilo that are useful for ex-core analysis with VERA. The commands for performing postprocessing are not shown here because they are not typical user capabilities. The following advanced processing capabilities are shown so that an interested user can contact one of the Shift developers for further details.

The most useful capability is being able to visualize a ray trace of the geometry. As stated in $\S 4$ and $\S 5$, this ray trace is dumped to an HDF5 file if the output_geometry is set to true. Along with this ray trace, the compositions used in the Shift calculation for each state point are also dumped to separate HDF5 files. These compositions can be used to enhance the coloring of the Python visualization. The geometry figures in $\S 5$ were produced using this Python visualization capability.

Figure 11 and Fig. 12 show examples of a plot by material using the ray trace and composition output files. Figure 13 and Fig. 14 show examples of using the ray trace by cell to interrogate different aspects of the ex-core geometry. 


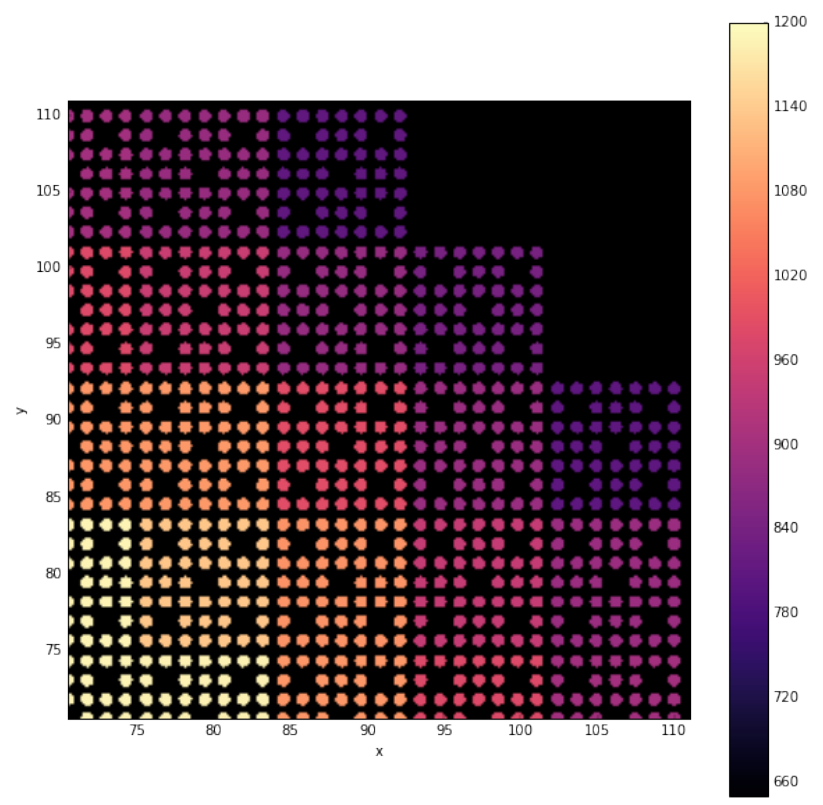

Figure 8. Example of assembly-averaged temperature homogenization in the fuel of progression problem 7 minicore [2].

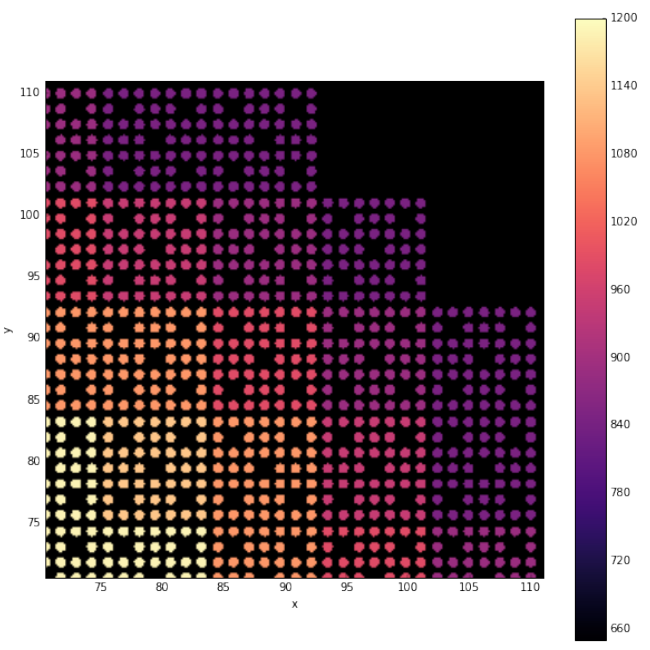

(a) Assembly ring-averaged

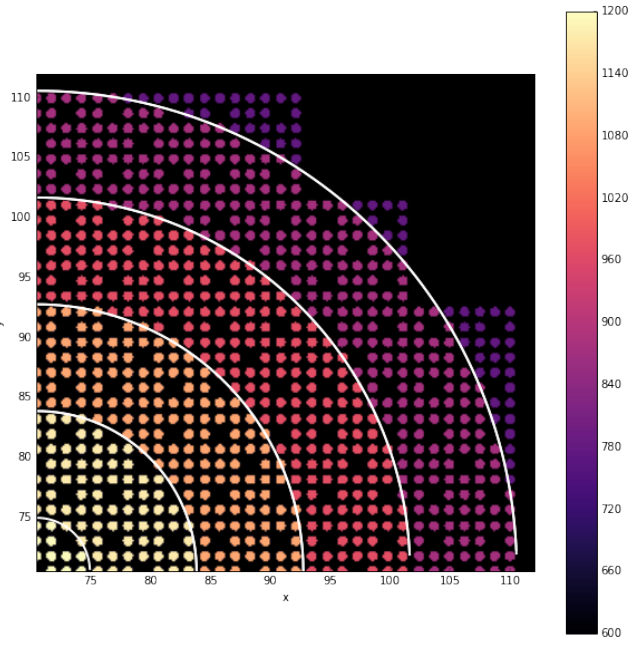

(b) Pin ring-averaged

Figure 9. Examples of ring-averaged temperature homogenization in the fuel of progression problem 7 minicore [2]. 


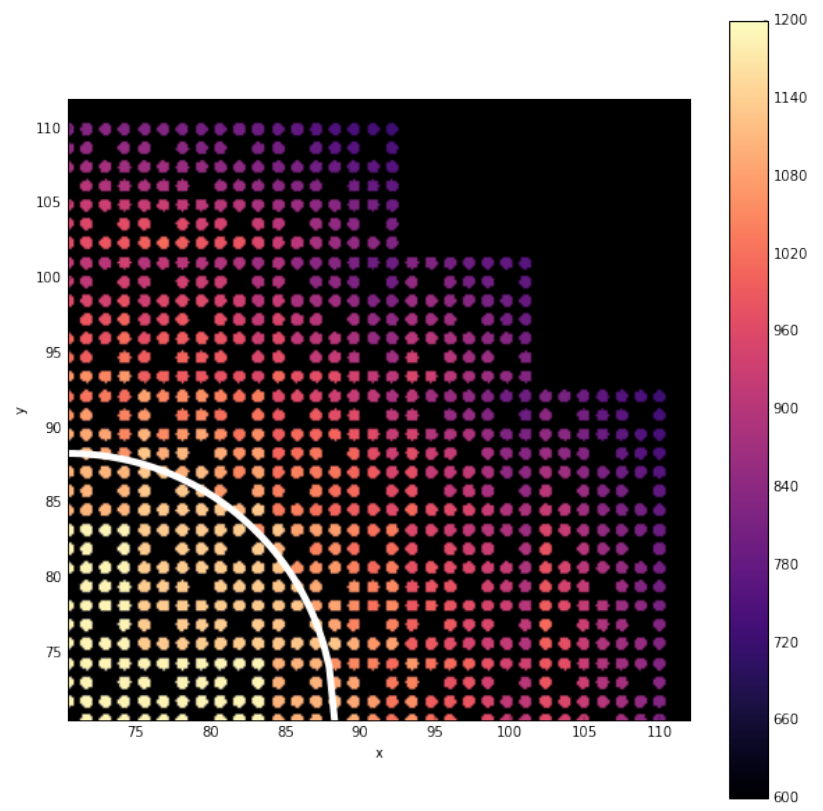

Figure 10. Example of explicit peripheral pins with assemblyaveraged temperature homogenization in the fuel of progression problem 7 minicore [2].

As mentioned previously, the fission source from MPACT used in the Shift calculation is dumped to the Shift HDF5 output file for every state. This source can also be visualized and interrogated with Shift postprocessing tools. Figure 15 shows an example of the spatial distribution of the fission source from progression problem 9 for the second state point plotted on top of the geometry.

Another postprocessing capability is visualization and inspection of the adjoint source and adjoint flux used in the CADIS calculation. Again, as mentioned in $\S 4$, the adjoint source is output to a separate HDF5 file, and the adjoint flux is output to the Shift HDF5 output file when running $v e r a \_t o \_s h i f t$ if the output_adjoint parameter is set to true. Figure 16 shows an example of an axial slice of the adjoint source used in the CADIS calculation of progression problem 9. Figure 17 shows an axial slice of the calculated adjoint flux for a particular energy group from this same calculation.

Finally, another useful capability is the visualization of the weight windows used in the Shift calculation when using CADIS. These weight windows are output to the Shift HDF5 output file if the output_ww flag is set to true. Figure 18 shows an axial slice of the weight windows for a particular energy group for progression problem 9. 


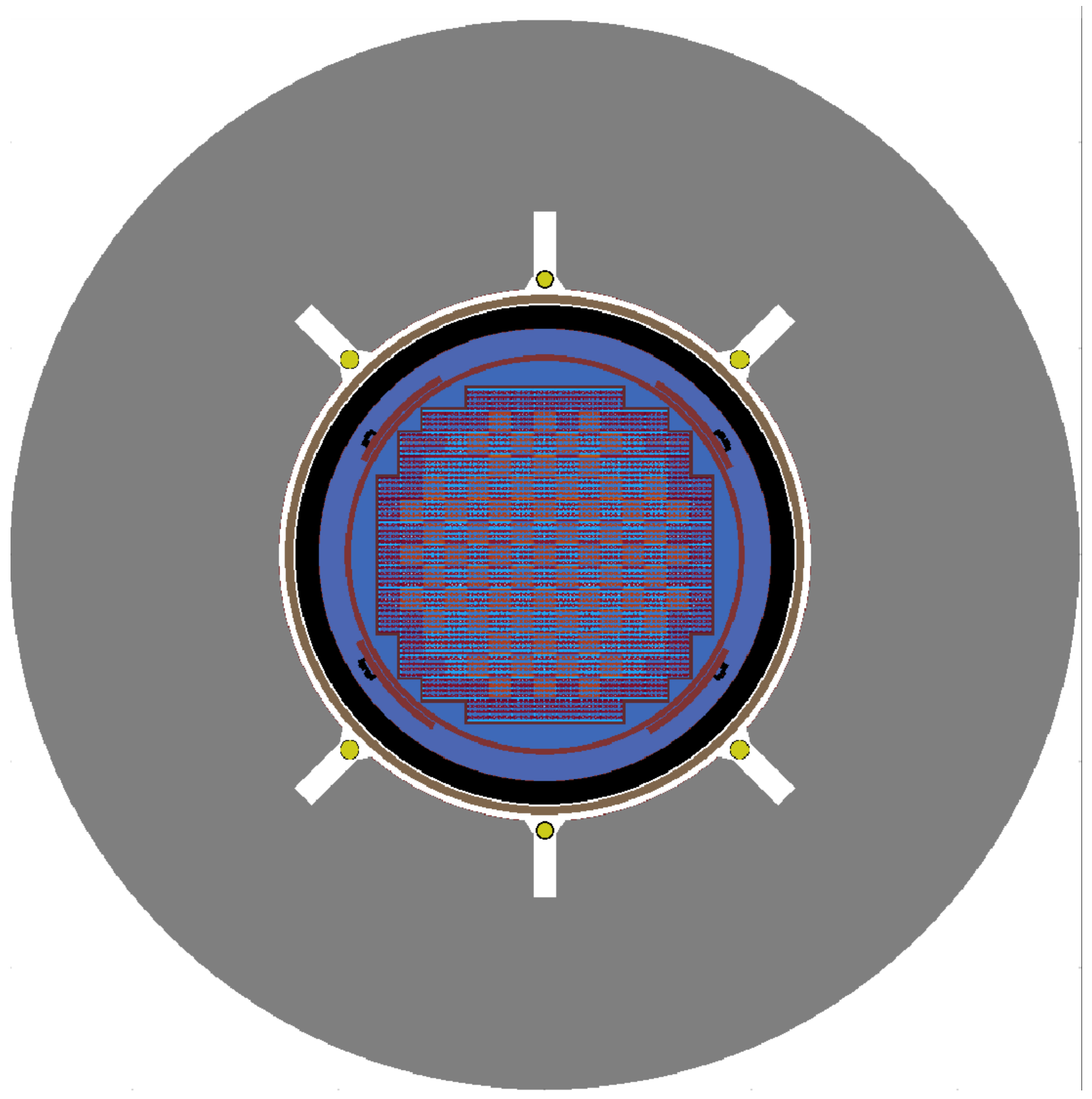

Figure 11. Full-core material ray trace $x-y$ slice at axial midplane (courtesy of Eva Davidson). The colors represent different material compositions. 


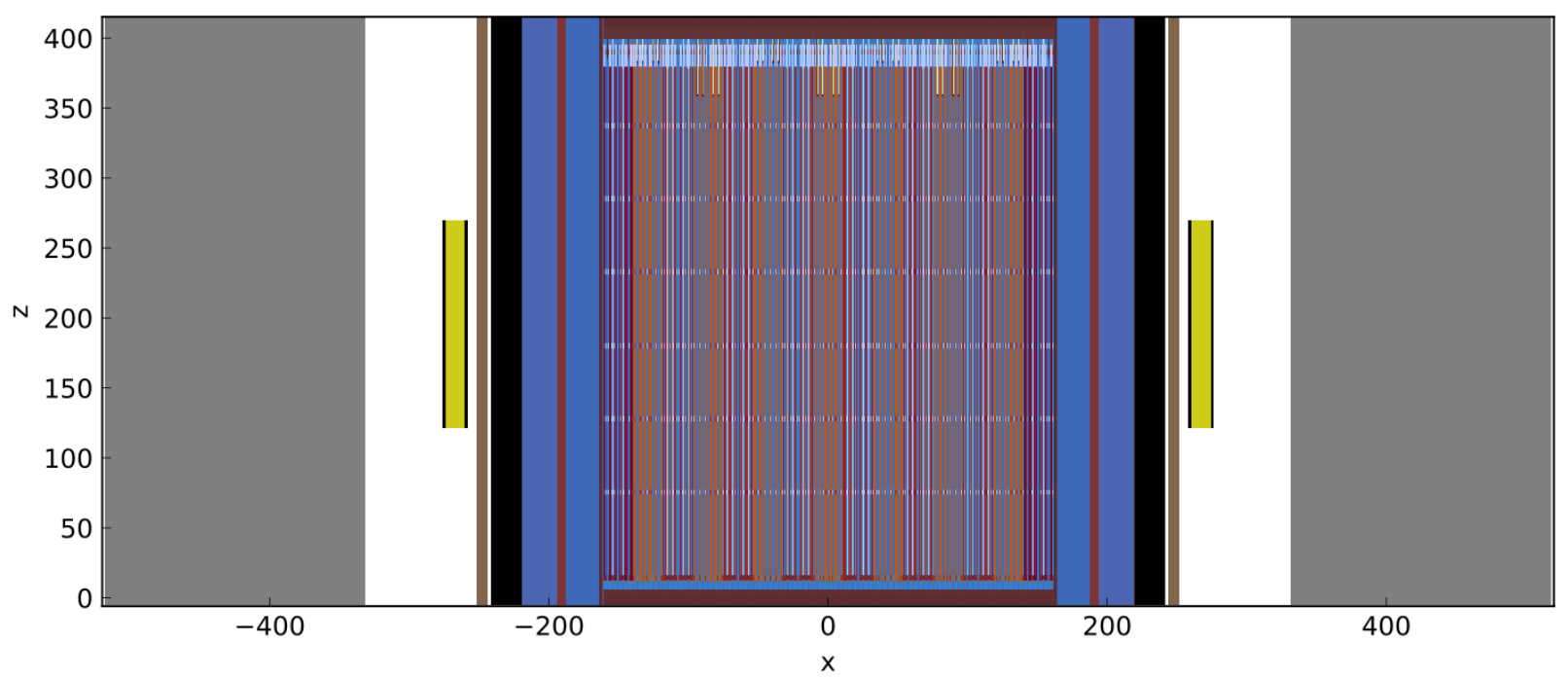

Figure 12. Full-core material ray trace $x-z$ slice at $y=0$ (courtesy of Eva Davidson). The colors represent different material compositions.

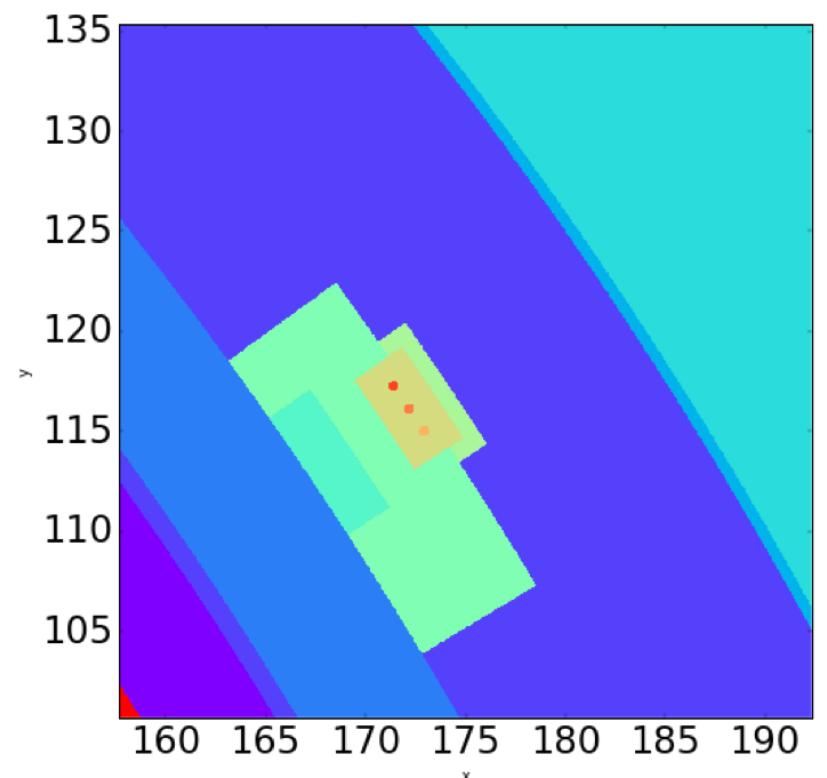

Figure 13. Irradiation capsules cell ray trace $x-y$ slice (courtesy of Eva Davidson). 


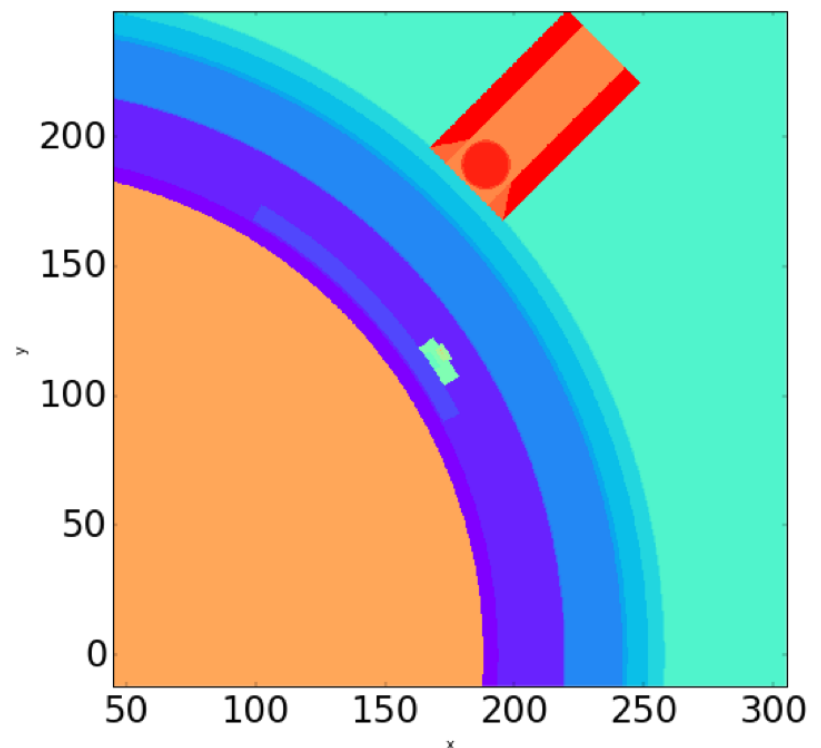

Figure 14. Source detector cell ray trace $x-y$ slice (courtesy of Eva Davidson). 


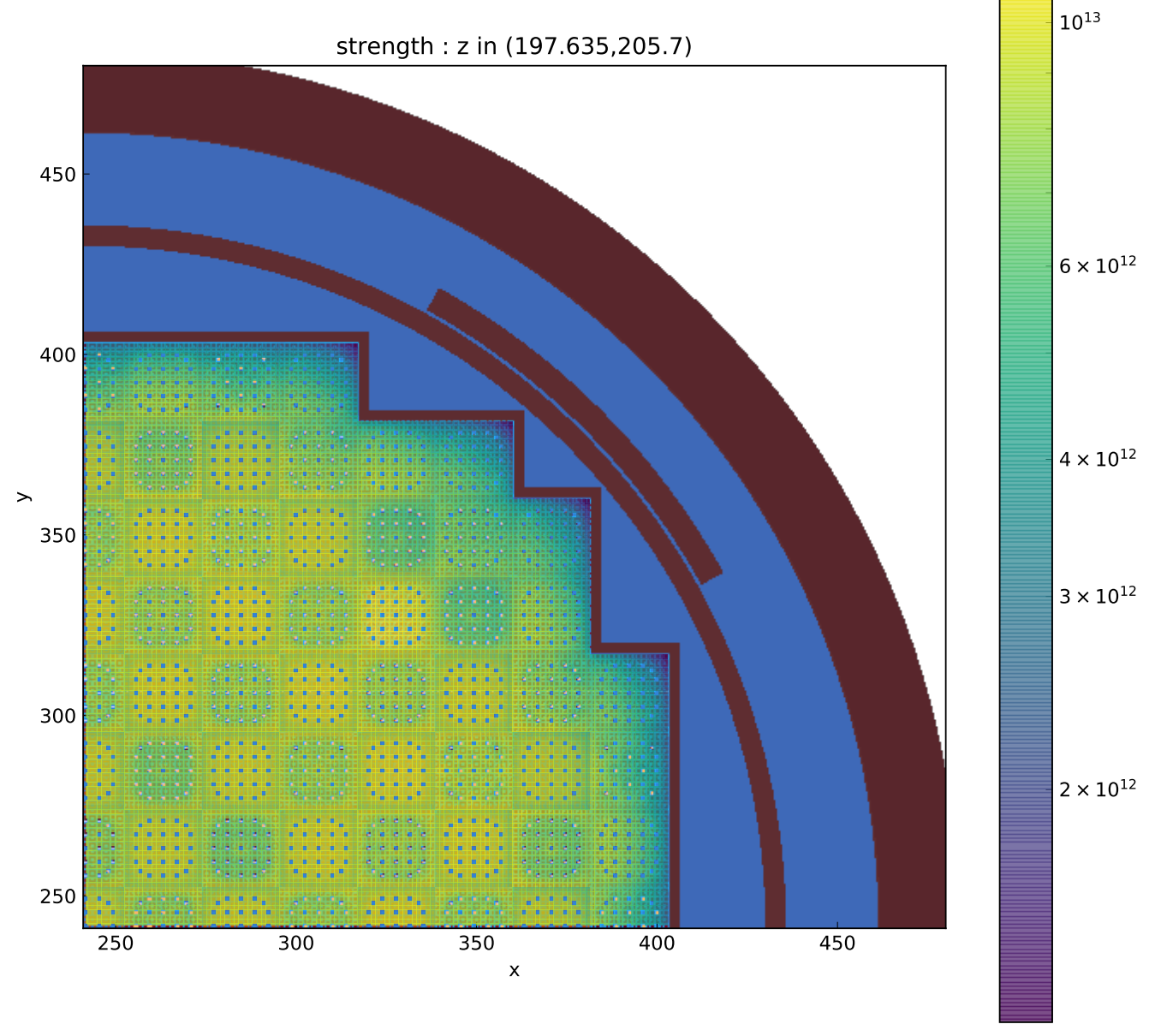

Figure 15. Progression problem 9 state 2 fission source $x-y$ slice. Fission source color scale is only applied in the assemblies. 


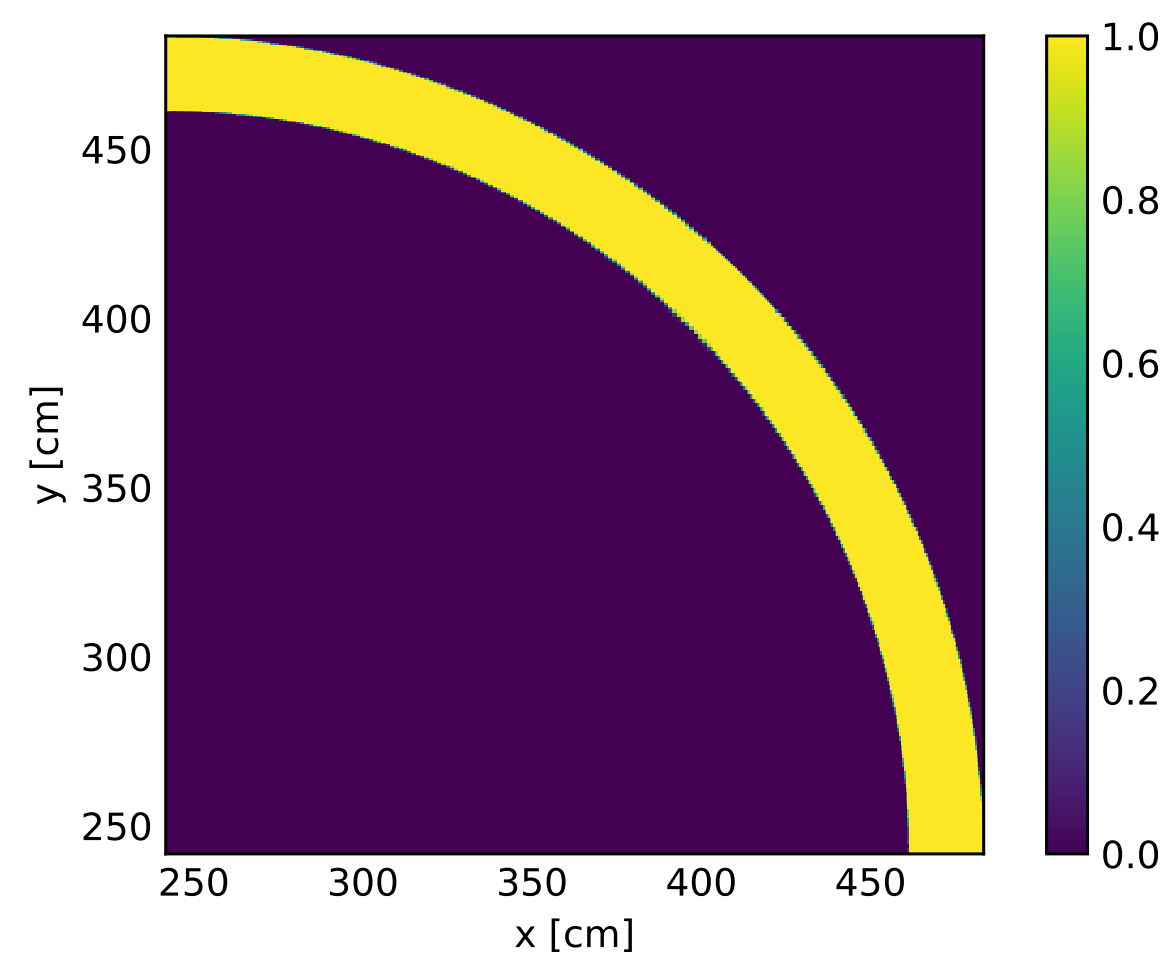

Figure 16. Progression problem 9 adjoint source $x-y$ slice. 


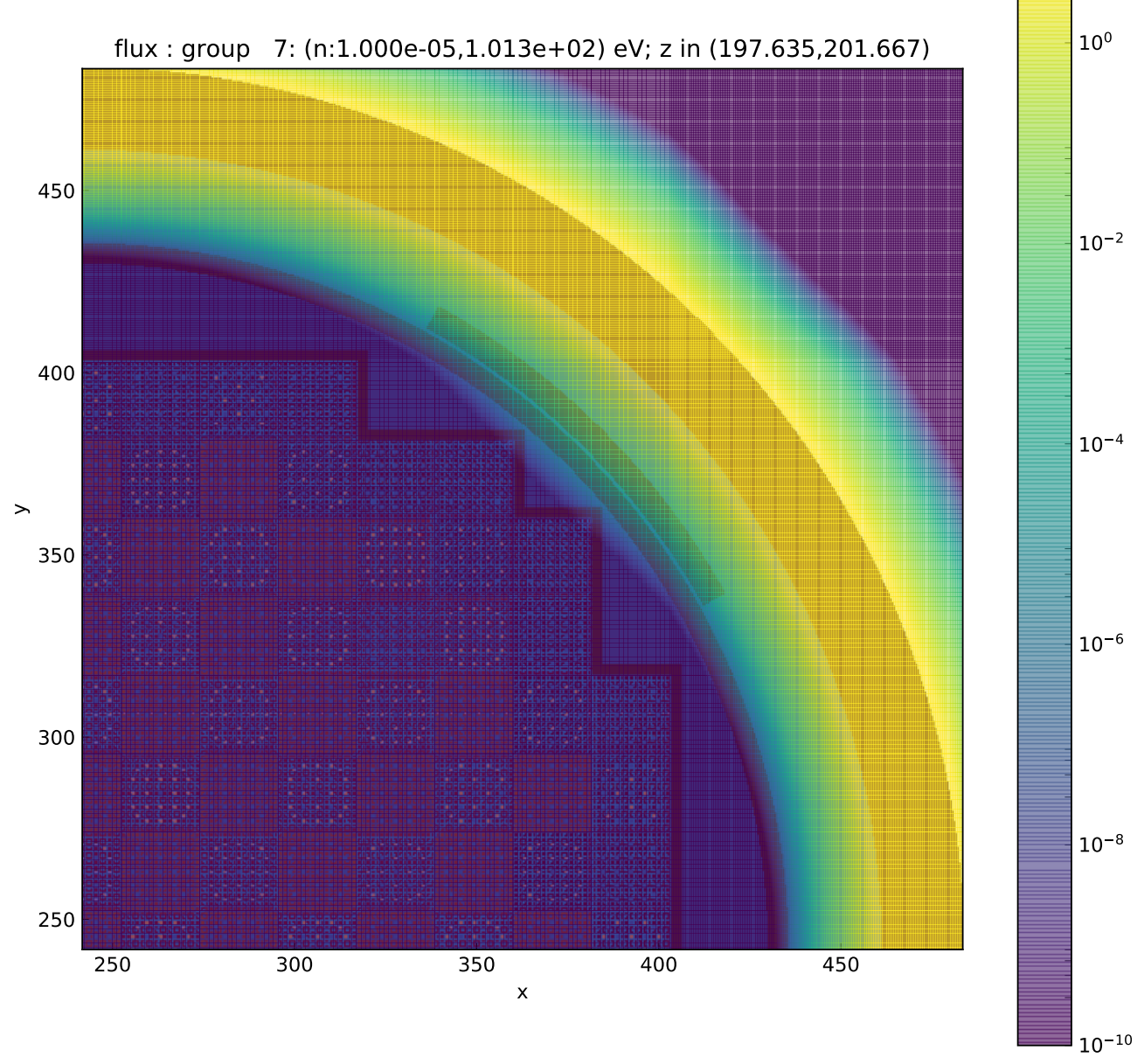

Figure 17. Progression problem 9 slowest energy group adjoint flux $x-y$ slice. The flux is superimposed on a colored material plot of the geometry but the color scale shown applies only to the adjoint flux. 


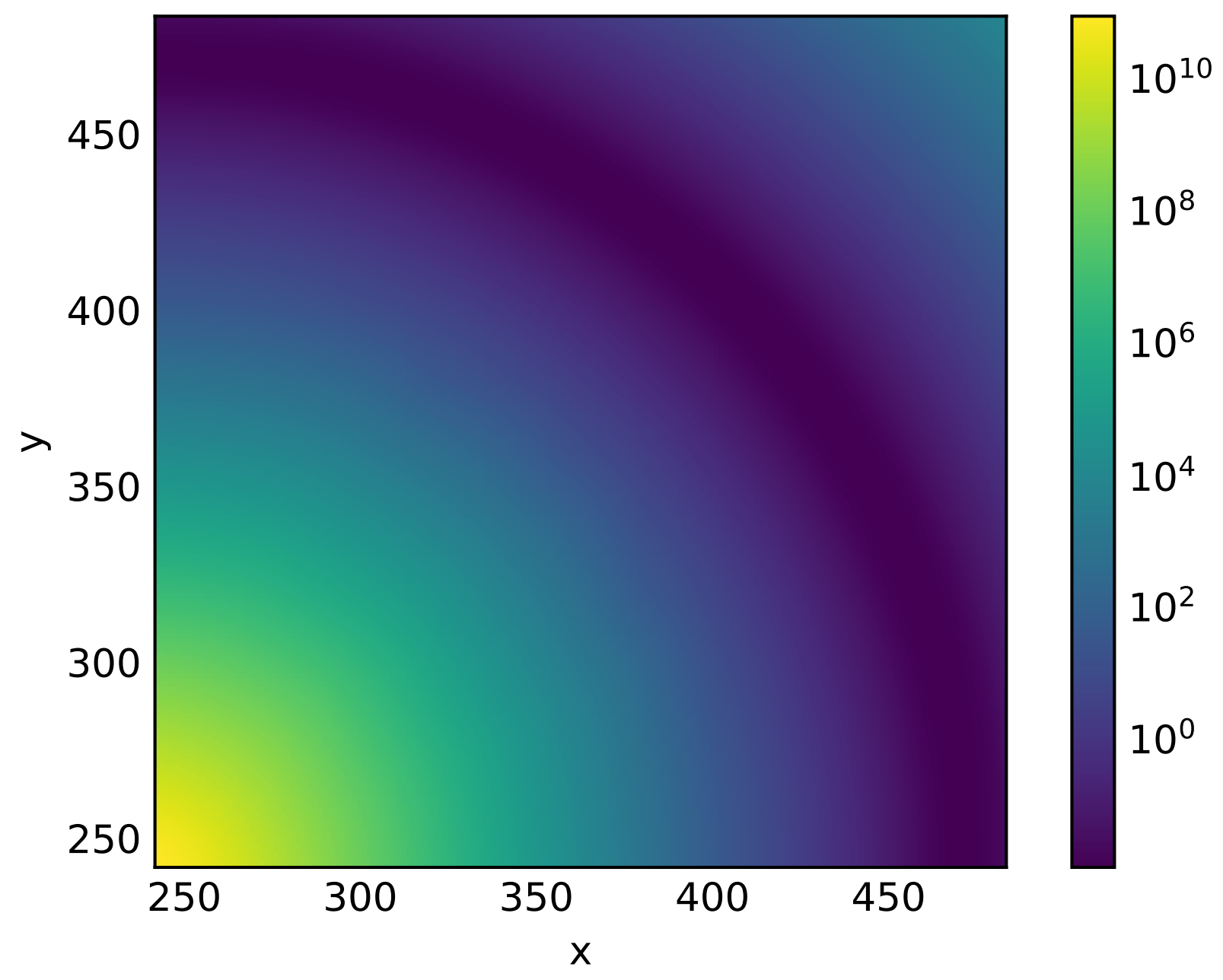

Figure 18. Progression problem 9 fastest energy group weight window lower bounds $x-y$ slice. 


\section{SUMMARY}

This manual describes the ex-core calculation capability of VERA. Shift and MPACT are coupled in parallel to perform these calculations. MPACT performs an eigenvalue calculation for each state point, and Shift performs either (a) an eigenvalue calculation for state point pin-power validation or (b) a fixed-source calculation to calculate vessel fluence and/or other ex-core quantities. In both cases, the complete state of the core is transferred to Shift.

VERA includes the following capabilities for ex-core calculations:

- node-based parallelism using domain replication;

- multistate;

- fission source coupling;

- pincell isotopic coupling;

- temperature (fuel, clad, coolant) and coolant density coupling;

- fluence in core barrel, core pads, vessel liner, and vessel wall;

- supplemental geometric and tally input for ex-core model features and tallies;

- automatic generation of supplemental ex-core input for bioshield and detectors; and

- eigenvalue, forward, and CADIS modes.

Although all of the transfers listed below can be enabled, the full coupling of pincell isotopic compositions, temperatures, and densities is very memory intensive and will not run for some quarter-core and all full core problems. 


\section{ACKNOWLEDGMENTS}

We would like to acknowledge the efforts of Andrew Godfrey, Eva Davidson, and Travis Lange in ex-core capability testing. We would also like to thank the efforts of Mark Baird for testing the build and installation of VERA with these ex-core capabilities. Finally, we would like to thank the MPACT development team at Michigan, with Brendan Kochunas as lead, for quick turnaround of bug fixes.

\section{REFERENCES}

[1] T. M. Pandya, S. R. Johnson, T. M. Evans, G. G. Davidson, S. P. Hamilton, A. T. Godfrey, Implementation, capabilities, and benchmarking of Shift, a massively parallel Monte Carlo radiation transport code, Journal of Computational Physics 308 (2016) 239-272.

[2] C. Stocking, An Investigation on the Impact of Temperature and Density Homogenization on Vessel Fluence Calculations, Thesis, North Carolina State University (2018).

[3] R. Montgomery, VERA tools and workflows, Technical Report CASL-U-2014-0054-001, Oak Ridge National Laboratory (March 2014).

[4] D. Jabaay, CASL MPACT User's Manual, Tech. Rep. CASL-U-2019-1873-000, CASL (July 2019).

[5] R. Salko, M. Avramova, A. Wysocki, J. Hu, A. Toptan, N. Porter, T. Blyth, C. Dances, A. Gomez, C. Jernigan, J. Kelly, CASL CTF User's Manual, Tech. Rep. CASL-U-2019-1885-000, CASL (March 2019).

[6] S. Palmtag, A. T. Godfrey, VERA Common Input User Manual, Tech. Rep. CASL-U-20140014-002, CASL (February 2015).

[7] J. C. Wagner, A. Haghighat, Automated variance reduction of Monte Carlo shielding calculations using the discrete ordinates adjoint function, Nuclear Science and Engineering 128 (2) (1998) 186-208.

[8] J. Wagner, D. Peplow, S. Mosher, FW-CADIS Method For Global And Regional Variance Reduction Of Monte Carlo Radiation Transport Calculations, Nuclear Science and Engineering 176 (1).

[9] A. Godfrey, R. Lee, VERAView User's Guide, Tech. Rep. CASL-U-2016-1058-000, Oak Ridge National Laboratory (March 2016).

[10] A. T. Godfrey, VERA Core Physics Benchmark Progression Problem Specifications, Tech. Rep. CASL-U-2012-0131-004, CASL (August 2014).

[11] CASL, CASL VERAIn public GIT Repository: VERA common input processor, https: //github. com/CASL/VERAin, accessed October 2019 (October 2019).

[12] S. R. Johnson, T. M. Evans, G. G. Davidson, S. P. Hamilton, T. M. Pandya, Exnihilo transport code manual, Tech. Rep. Release 6.2.0 (Dev), Oak Ridge National Laboratory (2017). 
[13] C. Stocking, T. Pandya, M. Avramova, Validation of MPACT Cross Section Library with Shift using VERA-CS, in: PHYSOR 2018: Reactor Physics paving the way towards more efficient systems, ANS PHYSOR Topical Meeting, American Nuclear Society, LaGrange Park, IL, 2018.

[14] T. M. Pandya, S. R. Johnson, B. Langley, R. A. Lefebvre, B. J. Marshall, Verification and validation of new SCALE sequence: CsasShift, Tech. Rep. ORNL/SR-2016/401, Oak Ridge National Laboratory (2016).

[15] T. M. Pandya, T. M. Evans, G. G. Davidson, S. R. Johnson, A. T. Godfrey, Shift verification and validation, Tech. Rep. CASL-U-2016-1186-000, Oak Ridge National Laboratory (2016).

[16] T. M. Pandya, S. R. Johnson, T. M. Evans, G. G. Davidson, S. P. Hamilton, A Massively Parallel Monte Carlo Radiation Transport Package, in: Joint International Conference on Mathematics an Computation, Supercomputing in Nuclear Applications and the Monte Carlo Method, 2015.

[17] E. D. Biondo, G. G. Davidson, T. M. Pandya, S. P. Hamilton, T. M. Evans, Deterministically estimated fission source distributions for Monte Carlo k-eigenvalue problems, Annals of Nuclear Energy 119 (2018) 7-22.

[18] S. R. Slattery, P. P. H. Wilson, R. P. Pawlowski, The Data Transfer Kit: A Geometric Rendezvous-Based Tool for Multiphysics Data Transfer, in: M\&C 2013, American Nuclear Society, 2013.

[19] X-5 Monte Carlo Team, MCNP-A General N-Particle Transport Code, Version 5, Volume II, Tech. Rep. LA-CP-03-0245, Los Alamos National Laboratory (2003).

[20] X-5 Monte Carlo Team, MCNP-A General N-Particle Transport Code, Version 5, Volume III, Tech. Rep. LA-CP-03-0284, Los Alamos National Laboratory (2003). 


\section{A. OMNIBUS MATERIALS TEMPLATE FILE}

Listing 17 shows the materials in the Omnibus template file included in VERA. These materials can be used with the bioshield and detector specifications in the VERA common input.

Listing 17. Omnibus materials template file in VERA.

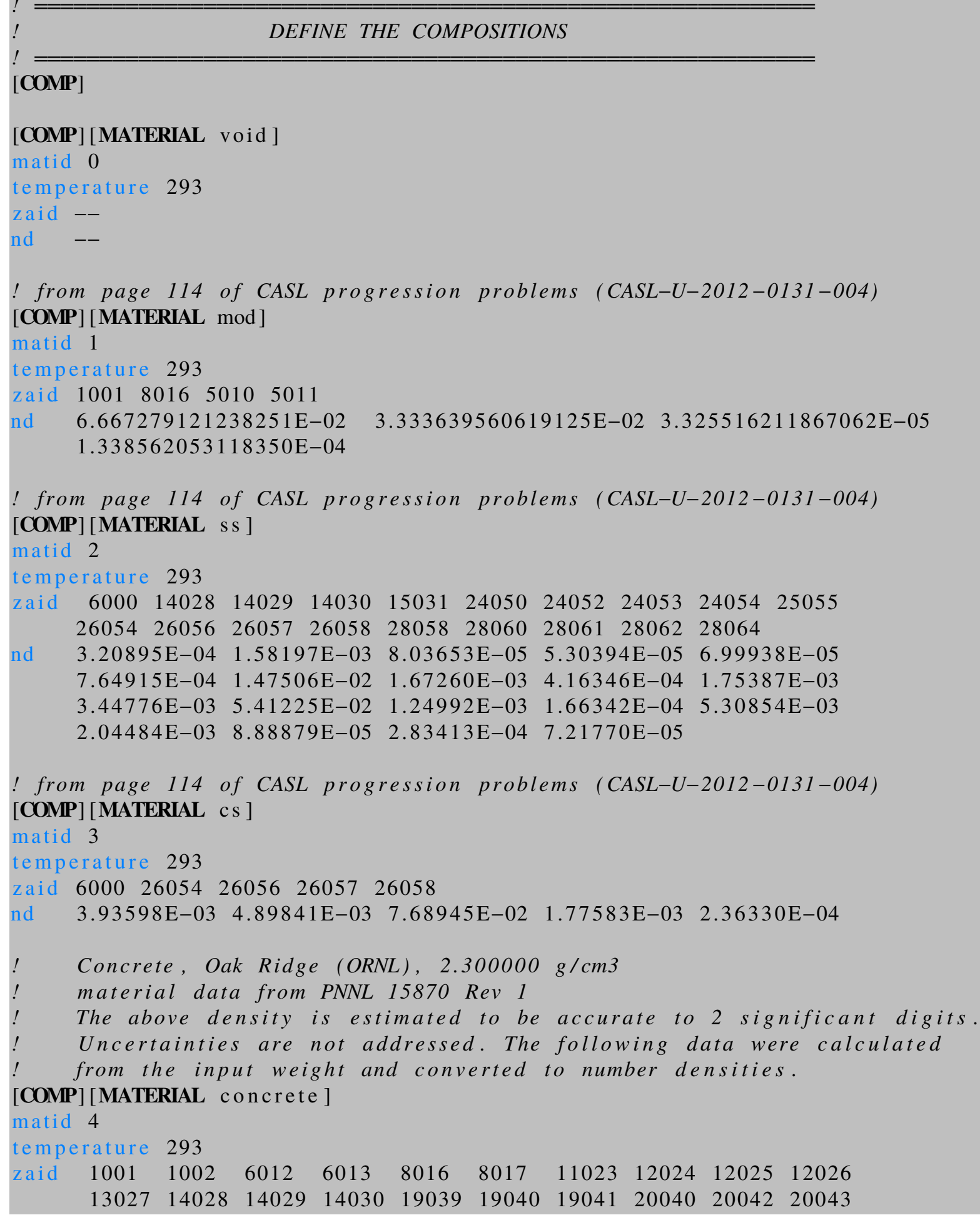


48

49

50

51

52

53

54

55

56

57

58

59

60

$\begin{array}{lllllll}20044 & 20048 & 26054 & 26056 & 26057 & 26058\end{array}$

nd $\quad 8.49996 \mathrm{E}-03 \quad 1.70019 \mathrm{E}-06 \quad 1.99790 \mathrm{E}-02 \quad 2.24257 \mathrm{E}-04 \quad 3.54247 \mathrm{E}-02$

$\begin{array}{lllllll}8.52237 \mathrm{E}-05 & 1.63354 \mathrm{E}-05 & 1.46966 \mathrm{E}-03 & 1.86056 \mathrm{E}-04 & 2.04848 \mathrm{E}-04\end{array}$

$\begin{array}{llllll}5.55961 \mathrm{E}-04 & 1.56827 \mathrm{E}-03 & 7.95785 \mathrm{E}-05 & 5.25422 \mathrm{E}-05 & 3.76029 \mathrm{E}-05\end{array}$

$4.03217 \mathrm{E}-09 \quad 2.71356 \mathrm{E}-06 \quad 1.07627 \mathrm{E}-02 \quad 7.21657 \mathrm{E}-05 \quad 1.55434 \mathrm{E}-05$

$2.32041 \mathrm{E}-04 \quad 2.10946 \mathrm{E}-05 \quad 1.12950 \mathrm{E}-05 \quad 1.77148 \mathrm{E}-04 \quad 4.09321 \mathrm{E}-06$

$5.40617 \mathrm{E}-07$

[COMP] [MATERIAL al ]

matid 5

temperature 293

zaid 13027

nd $\quad 6.02626 \mathrm{E}-02$ 


\section{B. DEFAULTS}

The following defaults are initialized in the SHIFT block. All defaults were given in Tables 1- 4 but these are the values explicitly set by the VERA pre-processing script. Any of these parameters can be overridden in the VERA input file, but this is not recommended for typical users.

\section{Listing 18. Default SHIFT block parameters.}

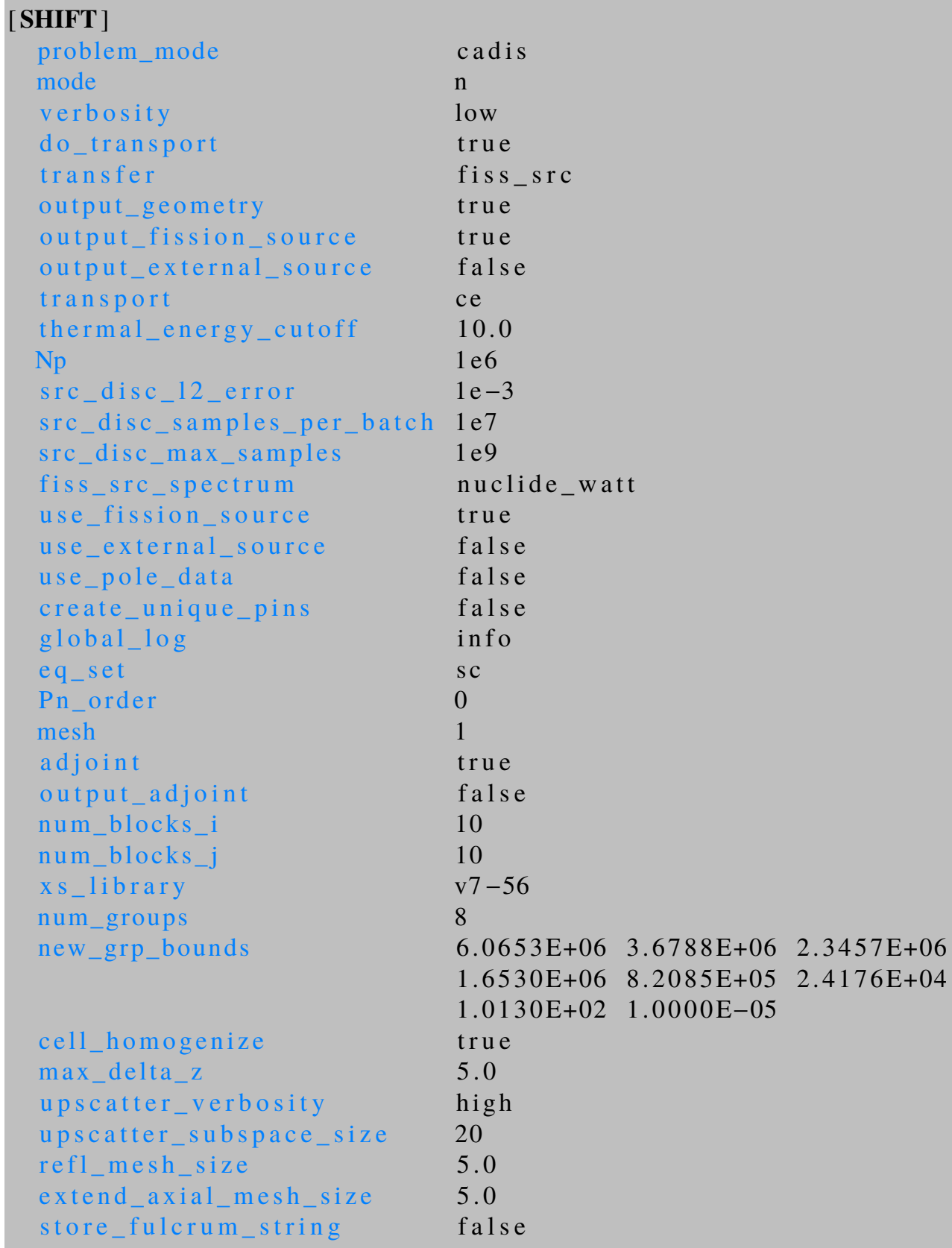




\section{GEOMETRY}

The Shift transport engine supports multiple geometric implementations, [1, 12] and VERA supports two of these geometric packages:

RTK (Reactor ToolKit): The RTK geometry is a highly optimized, simple geometric model consisting of nested boxes and cylinders. It is very effective for modeling incore pressurized water reactor (PWR) geometries, including the baffle. However, it has significant limitations treating detailed vessel models. Also, RTK cannot be extended to model detailed boiling water reactor (BWR) cores due to its lack of support for curved channel boxes.

GG (Exnihilo General Geometry): GG is a new general -purpose geometric modeling implementation in Shift [14]. It supports arbitrarily defined nested universes, arrays, and holes. Furthermore, many complex geometric shapes and quadratic surfaces are supported. GG can support detailed vessel models and user-defined ex-core features. It also supports all of the incore elements that RTK handles. The GG package can easily support any future VERA extensions for reactor models.

Even though RTK is optimized for PWR geometries, the GG package performs favorably on CE problems, as illustrated in Fig. 19. In multigroup problems, where geometric operations account for the majority of runtime, RTK is $\sim 2 \times$ faster. However, in CE problems, where physics operations consume the majority of run time, GG is only $17 \%$ slower than RTK.

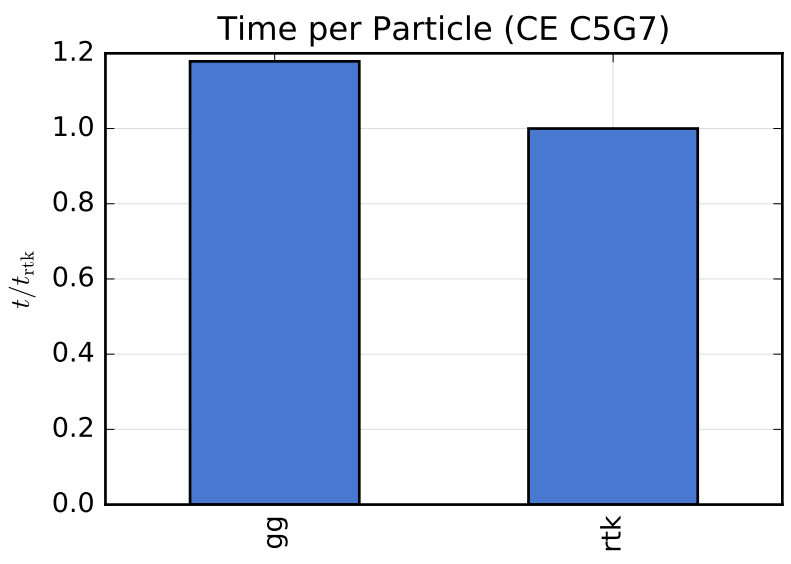

Figure 19. Performance of GG compared with RTK on reactor geometries with $\mathrm{CE}$ physics.

As mentioned in $\S 2$ and discussed in $\S$ D.1, the current implementation of VERA uses RTK for eigenvalue problems, GG for forward problems, and a combination of both for CADIS problems. There are no geometric impediments to using GG for eigenvalue problems; however, no current research interests exist to develop. The control flow to build the geometry in Shift through VERA is roughly equivalent for both models. The full incore model is built from the VERA input specification. A summary of the steps follows:

1. Convert XML input to a Teuchos: : ParameterList. 
2. Build the core data model that is a reactor-specific data model defining pins, inserts, compositions, and other light water reactor-specific features.

3. Using the lightweight core model, build persistent core metadata to store the reactor state.

4. Build the geometric model builder for either RTK or GG, depending on the problem mode.

5. In eigenvalue mode, use the geometric model builder to construct a complete incore reactor geometry for transport.

6. In forward mode, construct complete incore model out to the inner barrel or outer vessel radius. The ex-core builder then either adds the VERA vessel model, or it uses the supplemental ex-core model to define all geometric features outside the incore model.

7. Construct the problem decomposition using the model builder.

8. Construct the problem tallies using the model builder.

In CADIS mode, step 4 consists of meshing the RTK model for the deterministic adjoint calculation and building the GG geometry model for the MC calculation.

As described in step 3, VERA uses a metadata representation for the pincells and other core properties that is persistent throughout the simulation. The data transfer operations discussed in $\S$ D.2 update the metadata after each MPACT state point calculation. To allow for flexible updates from the VERA common input and to accommodate metadata updates, steps 2-8 are repeated for every state point.

In forward and CADIS problems, the geometry used for MC transport can be constructed two ways: (1) as a full-core model out to the outer vessel, as defined in the standard VERA input, or (2) as a full-core model out to the inner barrel radius or outer vessel radius, with ex-core model details provided by a supplemental input file. In the second case, the detailed core model can be translated to any position in space, which is a feature provided by the GG geometry. This mechanism can be used to drop a detailed core model into a larger scene that could include external core plant features not specified in the VERA input. The user must define tallies when using option 2 (these can be auto-populated if taking advantage of the automatic detector and bioshield option discussed in $\S 5.4$ ). For option 1, all tallies and geometric model options are specified by the standard VERA input. Figure 20 shows how the MPACT, Shift GG, and RTK geometry origins are defined by default in VERA.

A very useful feature through VERA is to dump axial slices of the geometry (from a ray trace in Shift) to an HDF5 file. This file can then be loaded into Python and visualized using processing utilities in Shift, as discussed in $\S$ 6.2. All of the input parameters supported for setting up a GG model can be found in the Shift manual for VERA [12]. An example of an ex-core GG model is given in $\S 5$. 


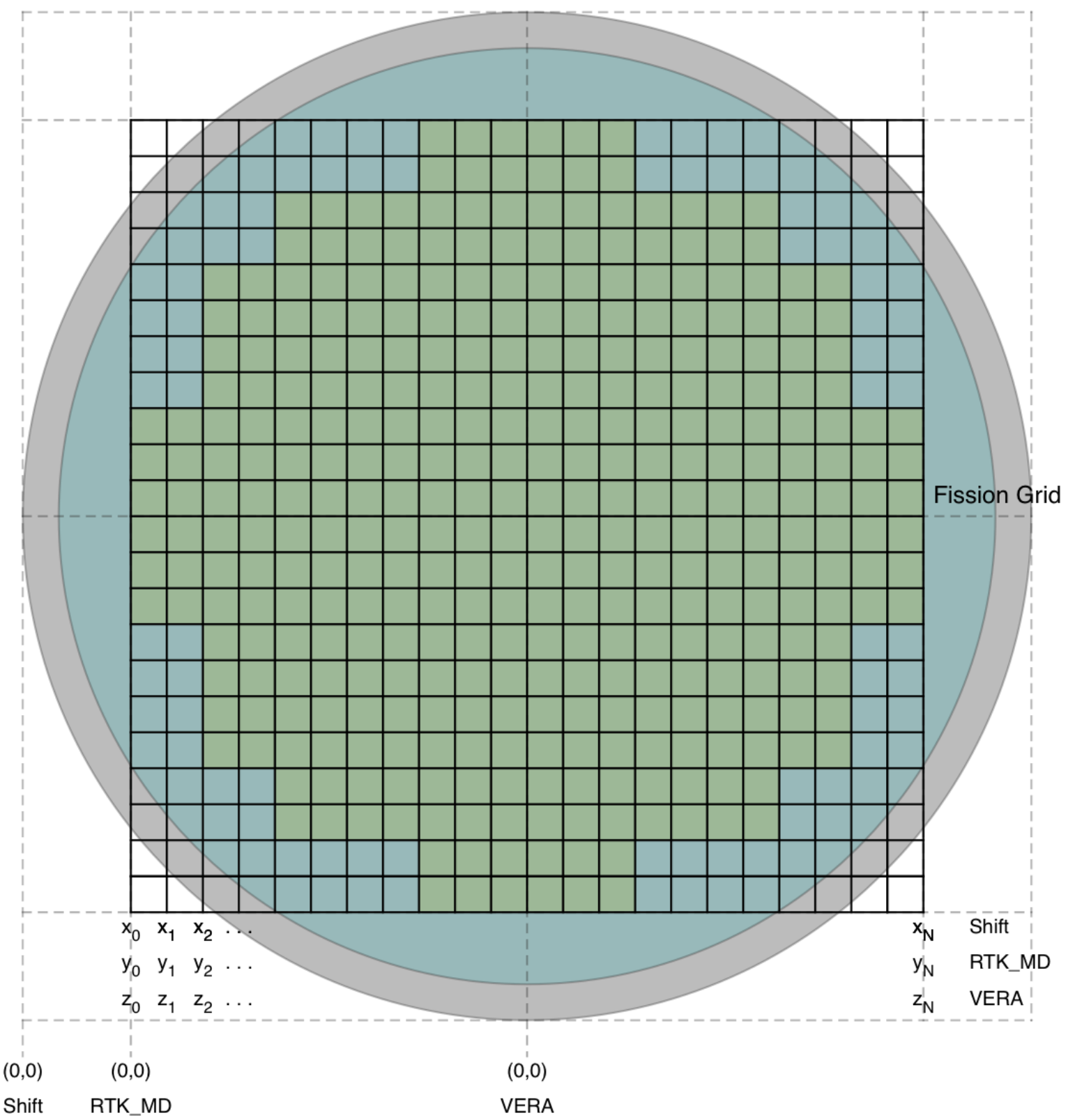

Figure 20. Geometry origin relationships in VERA between Shift GG, RTK, and MPACT. In this figure, VERA refers to MPACT. 


\section{METHODOLOGY}

This section presents details on how the VERA ex-core calculations are performed as implemented in the code itself, including methods of communication, and a more detailed explanation of what is transferred between MPACT and Shift.

\section{D.1. PACKAGE OVERVIEW}

The purpose of the vera_to_shift executable is to allow VERA users to set up and execute independent MC calculations at user-defined state points to validate and extend the modeling capabilties in VERA. Validation calculations typically consist of executing MC $k$-eigenvalue calculations at the current reactor state. The MC transport package used in VERA is provided by Shift. Validation of Shift for CASL use is described in Pandya 2016b [15]. Ex-core analyses are performed with Shift through VERA by running fixed-source calculations.

Shift provides novel transport algorithms and hybrid methods tailored for leadership-class computing platforms, in addition to multiple geometries, tallies, and physics $[1,16]$. For either vessel fluence or ex-core analyses, only one simulation is performed to calculate the quantities of interest by taking advantage of the coupling in VERA.

There are eight main areas to consider when discussing the capabilities of MC codes. A summary of these current ex-core capabilities in VERA are summarized in Table 11.

1. Energy treatment: The energy treatment used by Shift is CE physics for eigenvalue and forward problem modes. In CADIS mode, the deterministic $S_{N}$ adjoint calculation uses multigroup physics, and the forward MC calculation uses CE physics.

2. Geometric representation: In CADIS mode, the adjoint calculation meshes the RTK geometry up to the core barrel inner radius and then meshes the ex-core geometry using the GG geometry representation. The multigroup cross sections are then generated on this combined mesh.

3. Particle types: Only neutrons are transported for eigenvalue calculations, whereas Shift can follow neutrons or coupled neutron-photon transport for forward and CADIS calculations.

4. Material isotopic treatment: For both modes, Shift can use the depleted isotopics in the fuel pins.

5. Temperature treatment, For both modes, Shift can use the fuel, clad, and moderator temperature from CTF for each pincell.

6. Types of parallelism: VERA replicates the problem domain on all Shift processors requested.

7. Types of tallies: Different quantities of interest are tallied based on the problem mode. For eigenvalue calculations, the energy-integrated fission rate on a rectilinear mesh covering the pincells is calculated along with the eigenvalue. For forward and CADIS calculations, VERA can calculate the following: (1) the energy-binned fluence in a cylindrical mesh covering the barrel and vessel by default; (2) ${ }^{10} \mathrm{~B}$ or ${ }^{235} \mathrm{U}$ detector responses from the VERA input; or (3) any user-defined tally in the supplemental ex-core file. 
8. Types of sources: In a fixed-source problem, the MPACT neutron fission source is used as the neutron source for $n$ or $n-\gamma$ transport. The fission source from prompt $\gamma$ particles is not currently used from MPACT. When running a typical vessel fluence CADIS calculation, a detector response that is uniform in energy in the meshed vessel is used for the adjoint source, and the MPACT fission source is used for the MC forward calculation. For ex-core detector CADIS calculations, a user-defined detector response that is uniform in energy is meshed in the user-defined geometry cells for the adjoint source, and then the MPACT fission source is used for the MC forward calculation. As mentioned previously in $\S 2$, the user can specify whether to only use the spatial distribution from MPACT with a default ${ }^{235} \mathrm{U}$ Watt energy spectrum, a nuclide-dependent Watt spectra, or pull in the full spatial and energy spectra from MPACT for the fission source. In eigenvalue problems, the spatial distribution of the MPACT fission source is used as the initial source (eigenvector) for power iteration. This technique can significantly reduce the number of inactive iterations required for convergence, as shown in Biondo et al. 2017 [17] under specific circumstances.

Shift has additional capabilities not interfaced to VERA (e.g., MC multigroup energy treatment); a complete detailing of Shift's capabilities is available in Pandya et al. 2016a [1] and Johnson et al. 2017 [12]. Most modeling features are treated identically in both modes. For example, the temperatures are updated in all fuel pins for eigenvalue, forward, and CADIS problem modes if requested. 
Table 11. Ex-core capabilities of VERA.

\begin{tabular}{|c|c|c|c|}
\hline & \multicolumn{3}{|c|}{ Problem mode } \\
\hline Capability & Eigenvalue & Analog & CADIS \\
\hline Energy & $\mathrm{CE}$ & $\mathrm{CE}$ & $\mathrm{MG}$ and $\mathrm{CE}$ \\
\hline Geometry $^{a}$ & $\begin{array}{l}\text { RTK; only simple vessel } \\
\text { model supported }\end{array}$ & $\begin{array}{l}\text { GG; core automated } \\
\text { through VERA out } \\
\text { through barrel or vessel, } \\
\text { translation and additional } \\
\text { geometric features enabled } \\
\text { through supplemental } \\
\text { input }\end{array}$ & $\begin{array}{l}\text { meshed RTK for } S_{N} \\
\text { adjoint and GG for MC }\end{array}$ \\
\hline Particles & $n$ & $n$ or $n-\gamma$ & $n$ or $n-\gamma$ \\
\hline Isotopics & $\begin{array}{l}\text { depleted isotopics in fuel, } \\
\text { control rods, inserts, and } \\
\text { boron concentration via } \\
\text { MPACT }\end{array}$ & same as Eigenvalue & same as Forward \\
\hline Temperature $^{b}$ & $\begin{array}{l}\text { fuel, cladding, and } \\
\text { moderator temperatures } \\
\text { via CTF }\end{array}$ & same as Eigenvalue & same as Eigenvalue \\
\hline Parallelism & domain replicated & domain replicated & domain replicated \\
\hline Tallies & $\begin{array}{l}\text { rectilinear mesh (pincell) } \\
\text { fission, energy-integrated }\end{array}$ & $\begin{array}{l}\text { cylindrical mesh (vessel) } \\
\text { flux with energy bins } \\
\text { (through VERA input); } \\
\text { user-defined tally } \\
\text { definitions enabled } \\
\text { through supplemental } \\
\text { input (see [12]) }\end{array}$ & same as Eigenvalue \\
\hline Source & $\begin{array}{l}\text { spatial fission source via } \\
\text { MPACT; energy is either } \\
\text { sampled from a }{ }^{235} \mathrm{U} \text { Watt } \\
\text { spectrum, } \\
\text { nuclide-dependent Watt } \\
\text { spectra, or MPACT fission } \\
\text { spectrum }\end{array}$ & same as Eigenvalue & $\begin{array}{l}\text { adjoint source is uniform } \\
\text { energy response in vessel } \\
\text { or user-defined detector } \\
\text { response; MC source is } \\
\text { same as Eigenvalue }\end{array}$ \\
\hline
\end{tabular}

${ }^{a}$ See $\$ \mathrm{C}$ for detailed geometry descriptions.

${ }^{b}$ See $\S 6.1$ for experimental core temperature homogenization options. 


\section{D.2. COUPLING ALGORITHM}

The basic algorithm for solving forward and CADIS calculations with VERA is shown in Alg. 1 . First, a global message passing interface (MPI) communicator is established between MPACT and Shift. Shift and MPACT each run on their own sets of processors and on their own message passing interface (MPI) communicators. Next, the MPACT calculation is set up, along with the maps needed for the data transfer between MPACT and Shift. These maps use the Data Transfer Kit (DTK) [18] to handle the communication and data structures passed between MPACT and Shift. Next, MPACT launches a state point calculation. During this calculation, MPACT is run for the deterministic eigenvalue calculation, and CTF is run for the thermal hydraulics calculation.

Once a state point is complete in MPACT, the Shift eigenvalue, forward or CADIS calculation is launched. MPACT and Shift operate on independent domains. Thus, MPACT and Shift can execute different state points concurrently. Shift must wait on MPACT to finish an eigenvalue calculation for a state point before it can launch the forward or CADIS calculation of that state point. The vessel flux and some detector responses are communicated from Shift to MPACT. This communication is lagged by a state point.

The Shift calculation of a state point during the solve is either an $\mathrm{MC}$ forward calculation or a CADIS calculation, which performs an $\mathrm{S}_{N}$ adjoint calculation followed by an MC forward calculation. Shift uses the fission source transferred from MPACT as the source for the fixed source solve. The adjoint source for CADIS is either the detector response in the vessel, which is represented as uniform in energy, or the detector response in user-defined ex-core regions.

By default, Shift uses the short isotopic nuclide list from MPACT for the pins, control rods, and inserts of interest. The short nuclide list is given in $\S$ D.2.1. Shift can use the full isotopic nuclide list, but this introduces a large memory requirement for tracking all of the nuclides. Shift then runs the requested number of particle histories for the given state point and records the vessel flux tally (or ex-core detector tallies) in the HDF5 output file. The full cycle simulation is complete once MPACT completes the last state point and Shift completes the last requested forward calculation.

\section{D.2.1 Transfer Data}

As mentioned previously, the fission source and depleted isotopic compositions for each state point can be transferred between MPACT and Shift. VERA also couples temperatures and coolant densities to Shift with CTF via MPACT for each state point.

The following specified quantities can be transferred between MPACT and Shift:

- in-core neutron fission source from MPACT eigenvalue calculation;

- depleted isotopic compositions for pincells and inserts using the short list of tracked nuclides: ${ }^{234} \mathrm{U},{ }^{235} \mathrm{U},{ }^{236} \mathrm{U},{ }^{238} \mathrm{U},{ }^{16} \mathrm{O},{ }^{135} \mathrm{Xe}$, and ${ }^{10} \mathrm{~B}$;

- boron concentration in coolant;

- temperatures in fuel, clad, coolant of pincells and inserts; and

- density of coolant in pincells and inserts.

Data are transferred at pincell-centered points, where the term pincell refers to a 2D slice of a fuel pin, insert, or control rod. Shift determines the cell-centroids by interrogating an internal reactor 


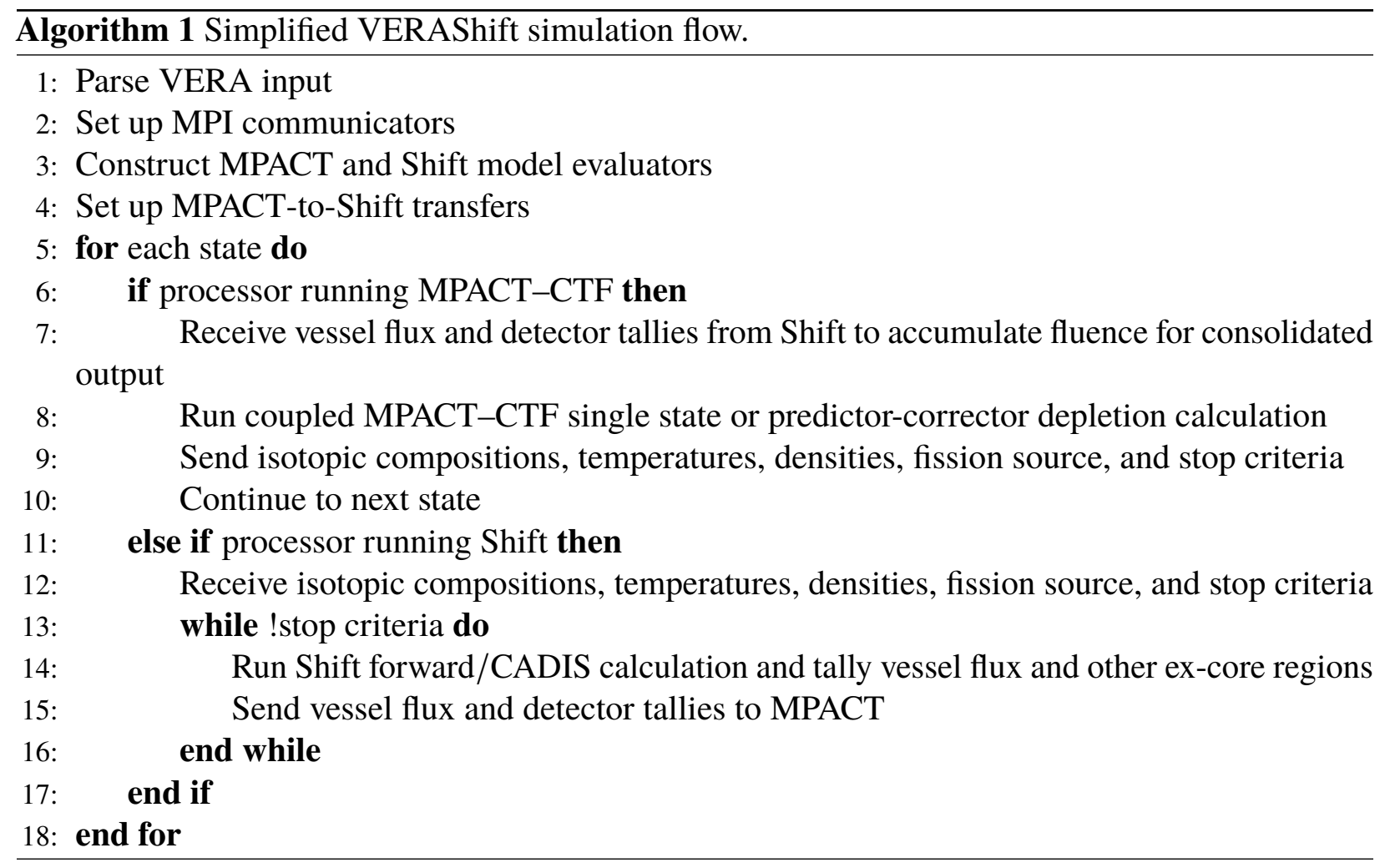

metadata model (see $\S \mathrm{C}$ ) and calculating the center point in each pincell axial location. This list of points is registered in DTK, which queries MPACT through the evaluator.

On the MPACT side, the isotopic compositions in the pincell identified at each point are homogenized over the containing pin volume, and a single set of nuclides is registered in DTK for transfer back to Shift because the same set of nuclides is tracked in each pin. The fission source and temperature transfers are handled in an analogous manner. DTK handles all the MPACT-to-Shift domain transfer mechanics.

For the fission source transfer, VERAShift can transfer either a nuclide-dependent fission source or a space- and energy-dependent source from MPACT. The spatially dependent fission source from neutrons calculated by MPACT is given by Eq. 4 :

$$
S_{c}=\sum_{i} v_{f, c}^{i} \Sigma_{f, c}^{i} \phi_{c} V_{c}
$$

where $i$ is a fissionable nuclide, $c$ is a pincell fuel region, $v_{f, c}^{i}$ is the number of neutrons produced from fission by nuclide $i, \Sigma_{f, c}^{i}$ is the macroscopic fission cross section of nuclide $i, \phi_{c}$ is the neutron flux in cell $c$ where the energy dependence has been omitted, and $V_{c}$ is the volume of cell $c$. The nuclide-dependent fission source is the default, and MPACT sends the spatial distributions (neutrons/s) for ${ }^{235} \mathrm{U},{ }^{238} \mathrm{U},{ }^{239} \mathrm{Pu}$, and ${ }^{241} \mathrm{Pu}$. Shift then samples the appropriate Watt energy spectrum based on the nuclide sampled for the fission source in a spatial location. The angular distribution of this fission source is always modeled as isotropic. The Watt energy spectrum is represented in Eq. 5, where $C$ is a normalization factor and the constants ( $a$ and $b$ ) used in Shift are given in Table 12.

$$
W(E)=C e^{-E / a} \sinh \sqrt{b E}
$$


Table 12. Nuclide Watt energy spectra constants used in Shift.

\begin{tabular}{llll}
\hline Nuclide & $\mathbf{a}(\mathrm{MeV})$ & $\mathbf{b}(1 / \mathrm{MeV})$ & Reference \\
\hline${ }^{235} \mathrm{U}$ & 0.96500 & 2.2900000 & MCNP5 manual (Cranberg spectrum) [19] \\
${ }^{238} \mathrm{U}$ & 0.88111 & 3.4005000 & MCNP5 manual [20] \\
${ }^{239} \mathrm{Pu}$ & 0.96600 & 2.8420000 & MCNP5 manual \\
${ }^{241} \mathrm{Pu}$ & 1.33196 & 0.0929657 & Manual fit to cross section data \\
\hline
\end{tabular}

With the space- and energy-dependent source from MPACT, Shift can either collapse the source in energy and sample a ${ }^{235} \mathrm{U}$ Watt energy spectrum for all nuclides or use the full nonseparable source distribution on the energy groups used by MPACT. Note that using the full energy distribution from MPACT is memory intensive and the provided group structure may not be appropriate for ex-core calculations. Previous research has shown that the assumption of a Watt energy spectrum is most likely reasonable for eigenvalue calculations [17]. The transferred source is the initial fission source for eigenvalue calculations in Shift and the fixed-source for forward and CADIS calculations.

Although VERAShift can transfer all of the quantities mentioned in this section, for most realistic problems this is memory intensive and is not realistic to do on the current computing architectures, especially considering the replicated nature of the materials in Shift. For problems larger than a few assemblies, it is strongly recommended that depleted isotopic compositions, temperatures, and densities not be transferred. For most ex-core calculations, transferring only the fission source is sufficient for the responses of interest. The ability to take advantage of domain decomposition in Shift through VERAShift is being implemented and will allow for transferring of all of the quantities discussed in this section. 